\title{
VOYAGER 1 IMAGING AND IRIS OBSERVATIONS OF JOVIAN METHANE ABSORPTION AND THERMAL EMISSION: IMPLICATIONS FOR CLOUD STRUCTURE
}

\author{
Rohert A. West 1 \\ Laboratory for Atmospheric and Space Physics \\ University of Colorado \\ Boulder, C0 80309 \\ Peter N. Kupferman \\ Jet Propulsion Laboratory \\ 4800 Dak Grove Drive \\ Pasadena, CA 91109 \\ and \\ Helen Hart
Laboratory for Atmospheric and Space Physics \\ University of Colorado \\ Boulder, CO 80309
}

1 Present address:

Earth and Space Sciences

Jet Propulsion Laboratory

4800 Oak Grove Drive

Pasadena, CA 91109

Manuscript Pages: 54

Figures: 38

Tables: 3 
Proposed Running Head: Jovian Cloud Structure.

Send correspondence and proofs to

Dr. Robert. A. West

MS 183-301

Jet Propulsion Lab

4800 Oak Grove Drive

Pasadena, CA 91109 
ABSTRACT

We make use of images from three filters of the Voyager 1 wide angle camera to measure the continuum reflectivity and spectral gradient near $6000 \AA$ and the 6190 \& hand methane/continuum ratio for a variety of cloud features in Jupiter's atmosphere. The dark "barge" features in the North Equatorial Belt have anomalously strong positive continuum spectral gradients suggesting unique composition, probably not elemental sulfur. We show methane absorption at unprecedented spatial scales for the Great Red Spot and its immediate environment, fór a dark barge feature in the North Equatorial Belt, and for two hot spot and plume regions in the North Equatorial Belt. Some small-scale features, unresolvable at ground-based resolution, show significant enhancement in methane absorption. Any enhancement in methane absorption is conspicuously absent in both hot spot regions with 5 un brightness temperature $255 \mathrm{~K}$. Methane ahsorption and 5 um emission are correlated in the vicinity of the Great Red Spot but are anticorrelated in one of the plume-hot spot regions.

We quantitatively compared methane absorption and simultaneous maps of 5 im brightness temperature to realistic cloud structure models which include multiple scattering at 5 im as well as in the visible. A curve in parameter space defines the solution to any observed quantity, ranging from a shallow atmosphere and thin $\mathrm{NH}_{3}$ cloud, to a deep atmosphere with a thick ammonia cloud. Without additional constraints, such as center-to-limb information, it is impossible to specify the $\mathrm{NH}_{3}$ cloud optical depth and pressure of a deeper cloud top independently. We also investigated variability in $\mathrm{H}_{2}$ quadrupole lines and found that the constancy of the 4-0 S(1) line equivalent width is consistent with the constancy of the methane $6190 \AA$ hand equivalent width at ground-based resolution, but the much greater variability of the 3-0 S(1) line 
is inconsistent with either the methane band or 4-0 S(1) line. In hot spot regions the $255 \mathrm{~K}$ brightness temperature requires cloud optical depth about 2 or less at $5 \mathrm{~mm}$ in the $\mathrm{NH}_{3}$ cloud layer. To be consistent with the observed 6190 A methane absorption in hot spot regions, the $\mathrm{NH}_{3}$ cloud optical depth in the visible is about 7.5 , implying that aerosols in hot spot regions have effective radii near 1 un or less. 
I. INTRODUCTION

The detailed shape of Jupiter's reflected sunlight spectrum between 0.2 and $1.0 \mathrm{~m}$ is dominated by absorption features due to methane gas and broadband continuum absorption by aerosols. Quantitative analyses of methane absorption bands by numerous investigators (Hess, 1953; Hunt, 1973; Michalsky et a1. 1974; Teifel, 1976; Wallace and Smith, 1977; Sato and Hansen, 1979; West and Tomasko, 1980; Lutz, et al. 1982), have contributed to a partial understanding of the cloud structure. Even a casual inspection of groundbased images in the strong 8900 \& band (Owen, 1969; West, 1979a) reveals structural differences between the Great Red Spot and its surroundings, differences between tropical and temperate zones, and a population of highaltitude aerosols in the polar regions and Equatorial Zone (EZ).

Other types of remotely sensed data also carry important clues about cloud structure and microphysical properties. These include absorption bands of other molecules, especially $\mathrm{H}_{2}$, center-to-limb variations (CTLV) and phase : angle variations in the continuum, CTLV and phase angle variations in the linear polarization, and thermal emission at $5 . \mathrm{m}$. The interpretation of these data is not straightforward due to a combination of factors such as the effects of multiple scattering, uncertainties associated with various poorly constrained parameters, the simplifying assumptions introduced to make the problem tractable, and errors in the observations and supporting laboratory data. It has been the rule rather than the exception that analyses of two independent sets of data by independent investigators have resulted in mutually exclusive model parameters.

As an important illustration of this point, let us examine the proposed distribution of chromophores by comparing models hased on 5 um emission (Terrile and Westphal, 1977; Owen and Terrile, 1981) with models based on 
reflected sunlight in the visible and near-infrared (Sato and Hansen, 1979; West, 1979b; Smith and Tomasko, 1984). Owen and Terrile stress the correlation between cloud colors and $5 \mathrm{~mm}$ emitted intensity (the colored clouds are associated with higher 5 um intensity than are: the 'white' clouds) and assign physical temperatures, and therefore pressure levels, to the clouds. In their model, we are seeing strongly absorbing aerosols near the 2.4 bar level in the 'brown barges' which reside in the North Equatorial Belt (NEB) near $14.5^{\circ}$ latitude, and we can see down to about 2 bars in the light brown or 'tawny' clouds of the belts, and to about 4 bars in the 'blue-grey' areas in the NEB. Sato and Hansen (1979), West (1979b), and Smith and Tomasko (1984) arrive at a contradictory conclusion from analyses of absorption data in the near-infrared and polarization data from Pioneers 10 and 11 . The reflected sunlight data indicate little structural difference between belts and zones. The belt/zone difference in polarization can be explained entirely by the difference in single scattering albedo of the aerosols. In both belt and zone the 'ammonia' cloud top is near 320 mbar and its optical depth is at least 2 .

West and Tomasko (1980) suggested two ways to reconcile the 5 un and visible data. If the aerosols in belt regions have a mean radius near $1 \mu \mathrm{m}$, their extinction cross-section at $5 \mathrm{~mm}$ would be much less than in the visible and near-infrared, permitting 5 um radiation to escape while providing a significant source of scattering in the visible. If the Owen and Terrile assertion that we see down to a few bars in some regions is correct, the low spatial resolution of the ground-based absorption data may mask these regions if their spatial scale is smaller than the resolution element (about 1 arc-sec in the images of West (1979a)). An argument has also been made that the 5 um data were not obtained simultaneously with other kinds of data and temporal 
variability may account for the differences. At the very least, the Voyager 1 data are capable of resolving both the spatial scale and temporal variability issues since the imaging data have very high resolution and the Voyager Infrared Radiometer and Interferometer Spectrometer (IRIS) instrument made simultaneous measurements of $5 \mathrm{~km}$ intensity.

The 6190 \& (methane) filter on the Voyager 1 wide angle camera is particularly well suited to the task of searching for holes in the clouds. The absorption is weak loptical depth unity for methane absorption plus Rayleigh scattering from $\mathrm{H}_{2}+$ He occurs near the 10 bar level), and penetration of a significant fraction of the photons to the 4 bar level will be readily apparent in the reduced methane/continuum ratio image. High spatial resolution images of methane absorption allow, for the first time, an investigation of upwelling-downwelling diagnostics at length scales relevant to dynamical regimes which include small scale convection and inertia-gravity waves. The interpretation of spatial and temporal variations of $\mathrm{H}_{2}$ and $\mathrm{CH}_{4}$. absorptions observed from the ground (Carleton and Traub, 1974; Hunt and Bergstralh, 1977; Cochran and Cochran, 1980 and 1983) is advanced both by the ability to identify the cloud morphology associated with the variability, and to deconvolve the depth of the absorption and the size of the ground-based resolution element.

Care must be exercised in extracting photometric data from a vidicon detector, and we describe in the following section the methods, calibration, and uncertainties associated with this effort. The derived methane/continuum ratio images are shown in Section III, and the products are interpreted in terms of cloud structure models. The methane/continuum images are compared to contour maps of $5 \mathrm{~m}$ brightness temperature and $\mathrm{H}_{2}$ para fraction. The conclusions and outstanding issues are summarized in Section IV. 


\section{I1. OBSERVATIONS AND DATA REDUCTION}

Our goal is to derive a measure of the strength of the 6190 \& methane absorption from calibrated data number (DN) values in the Voyager 1 images. Combined filter-optics-vidicon response functions for three filters used in this study are shown in Fig. 1, along with a spectrum of Jupiter's North Tropical Zone (NTrZ) taken in early 1979 in support of the Voyager encounters (Cochran et al., 1981), and methane absorption coefficients (Giver, 1978). The filter curves are tabulated by Danielson et al. (1981) who discuss the photometric characteristics of the Voyager cameras.

Let us defer the discussion of photometric accuracy of the camera and assume that the DN values are linearly proportional to integrated radiance. Following Danielson et al. (1981), the wavelength averaged intensity is

$$
I=\frac{-\int I_{\lambda} R_{\lambda} d \lambda}{\int R_{\lambda} d \lambda}
$$

where $R_{\lambda}$ is the relative response shown in Fig. 1 , and $I_{\lambda}$ is the intensity. The quantity $1 / F$, ( $\pi$ times the intensity divided by the incident solar flux, $\pi \mathrm{F}$ at $5.2 \mathrm{AU})$ is given by

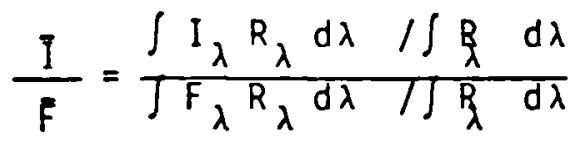

The Voyager filter responses cover a broad spectial range. Aerosols absorb and scatter radiation at continuum wavelengths, and their spectral characteristics will influence the $D N$ values. We can remove spectral 
reflectivity gradients caused by aerosols, to first order, by using the green and orange filters to estimate a continuum $I / F$ at the wavelength of the methane filter. A Taylor series expansion for the continuum times a curve of growth function for methane ahsorption serves to characterize the spectrum rather well in the wavelength range $4500 \AA<\lambda<6350 \AA$.

$I / F=\left[I / F_{0}+\left(\lambda-\lambda_{0}\right) \cdot \partial / \partial \lambda\left(I / F_{C}\right)+0\left(\lambda-\lambda_{0}\right)^{2}\right] \cdot f(k(\lambda))$

From a previous study (Fig. 6 of West, 1979b) a curve of growth function which fits the weak methane bands is $f(k(\lambda))=\left(1-D_{k}(\lambda)^{0.93}\right)$, and $D$ is a constant determined by the fitting proceedure and is proportional to the $6190 \AA$ equivalent width.

We define $\lambda_{0}$ as the effective wavelength of the orange filter. If terms in the continuum reflectivity of order $\left(\lambda-\lambda_{0}\right)^{2}$ and higher are small compared to constant and linear terms, and if we can neglect methane absorption in the orange and green filters, we can solve for the continuum spectral reflectivity gradient $\partial / \partial \lambda(I / F)_{C}$ at $\lambda 0$, and the filter averaged methane/continuum ratio, ${ }^{\mathrm{R}} \mathrm{CH}_{4} \cdot$

$I / F_{0}=\alpha D N($ Orange $)$

$\partial / \partial \lambda\left(I / F_{C}\right)=\alpha[D N($ Orange $)-D N($ Green $)] /[\lambda($ Orange $)-\lambda($ Green $)]$

$\mathrm{R}_{\mathrm{CH}_{4}}=\alpha \mathrm{DN}$ (Methane) $/\left[\alpha \mathrm{DN}(\right.$ Orange $)+(\lambda($ Methane $)-\lambda($ Orange $\left.)) \cdot \partial / \partial \lambda\left(\mathrm{I} / \mathrm{F}_{\mathrm{C}}\right)\right]$

ミmethane/continuum ratio.

$\alpha=$ absolute calibration constant 
and the solar weighted effective filter wavelengths are

$$
\lambda \text { (Green) }:=\frac{\int \lambda F_{\lambda} R_{\lambda} d \lambda}{\int F_{\lambda} R_{\lambda} d \lambda}=5703 \AA \text {, }
$$

$$
\begin{aligned}
& \lambda(\text { Orange })=6046 \AA, \\
& \lambda(\text { Methane })=6208 \AA .
\end{aligned}
$$

We use the above equations to determine $\mathrm{R}_{\mathrm{CH}_{4}}$, recognizing that some error is introduced by neglecting terms of order $\left(\lambda-\lambda_{0}\right)^{2}$ and higher, and that methane absorption in the orange filter is not negligible. In the analysis section we describe how methane absorption in the orange filter is included. We can quantify the error caused by high-order terms in the Taylor expansion by fitting the ground-based spectra of Cochran et al. (1981) and Woodman et al. (1979) by a nonlinear least squares method to the functional form given by equation (1). We fitted four spectra (EZ, NEB, NTrZ, and South Tropical Zone, STrZ) from Cochran et al., and a single NTrZ spectrum from Woodman et al.. Of these, the term of order $\left(\lambda-\lambda_{0}\right)^{2}$ decreases $\mathrm{R}_{\mathrm{CH}_{4}}$ by factors ranging from 0.995 for the NTrZ to 0.991 for the EZ. Since we calibrate $\mathrm{R}_{\mathrm{CH}_{4}}$ with ground-based spectra of the NTrZ, the error in $\mathrm{R}_{\mathrm{CH}_{4}}$ due to a nonlinear continuum will be 0.0 in the NTrZ and about $0.4 \%$ in typical belt regions. This error contribution is small compared to the uncertainty in the photometry from other sources discussed below.

We found that equation (1) including terms of order $\left(\lambda-\lambda_{0}\right)^{2}$, and the curve of growth function described above provide a very good fit to the Woodman et al. spectrum, but did not fit the shape of the $6190 \mathrm{~A}$ band in the 
spectra of Cochran et a1.. The 6190 \& bands in their spectra are systematically shifted to shorter wavelengths, and the Full Width Half Max (FWHM) to depth ratio is greater than for the hest-fit analytic spectra. For this reason we believe the equivalent widths they report. are too large, while we found the equivalent width for the Woodman et al. NTrZ spectrum to be larger than those authors report. We recommend fitting observed spectra with equation (1) as a way of discovering possible systematic errors and measuring the equivalent widths accurately by taking the guess work out of finding the continuum level:

Equations (2)-(4) can be used only if (1) the DN value is proportional to an absolutely calibrated integrated intensity, and the constant of proportionality, $\alpha$, is known, (2) picture elements (pixels) of the green, orange and methane frames must be mutually co-registered to high accuracy (typical residual root mean square (rms) registration errors are \pm 1 pixel in $1000 \times 1000$ pixel frames), and (3) all three frames must be shuttered within a small enough time interval to insure that the photometric expression of variations in lighting and viewing angles from one frame to the next are small compared to variations in the photometry due to methane ahsorption. Only a small subset of the Voyager 1 images satisfies the last criterion, and we list in Table I the images chosen for this study. A slightly larger number of images was initially chosen but several were rejected due to their inability to satisfy criterion (2). Additional images exist but our limited resources did not allow us to include them in this study.

The first six images in Table I were obtained during the inbound phase of the encounter just after the time Jupiter's angular diameter exceeded the $3.18^{\circ}$ wide angle frame field of view. These were selected to provide, as much as possible, a global view of methane absorption. No useful methane images 
were shuttered before this time period. Only two sets of three images for this time period satisfy criteria (1)-(3) above, these fall far short of providing global coverage. Three sets of images. (FDS 16373.37-16379.52) have significantly higher spatial resolution than the first two sets and cover much smaller areas. The first and second sets were obtained as part of a raster scan map of the IRIS field of view over two 5 um hot spot regions in the North Equatorial Belt (NEB), and the third is a map of the Great Red Spot (GRS).

Vidicon cameras traditionally have been poor photometric devices. Anyone attempting to extract photometric data from them is advised to pay attention Young's (1974) indictment of the Mariner 9 experience. Vidicons are nonlinear, both photometrically and geometrically, they can be temporally unstable, and they can display hysteresis, or residual image, effects. Vidicon technology has advanced since the Mariner 9 days, and the highest quality detectors were selected for the Voyager cameras, so the Voyager data are apparently of much higher photometric quality than are the Mariner 9 data. Danielson et al. (1981) describe the radiometric calibration of the Voyager images, and the reduction of the images in Table I is based on their work. We will briefly describe the data reduction method and the uncertainties.

The photometric and geometric calibrations were performed at the Image Processing Laboratory (IPL) of the Jet Propulsion: Laboratory using the VICAR imaging processing system (Castleman, 1979). The data were originally encoded to eight bits and repacked to 16 bit pixels during the calibration to avoid roundoff errors. Geometric distortion, inherent in all vidicons, was minimized through a transformation (IPL program GEOMA) determined from the apparent positions of reseaus (fiducial markings) in each image and their actual positions taken from the measurements of the vidicon faceplate during 
ground instrument calibration (Voyager Calibration Document, JPL 618-802, 1979). The raw $800 \times 800$ pixel images are transformed to $1000 \times 1000$ pixel images by GEOMA. Data numbers that differed significantly from their neighbors due to transmission (spacecraft to ground) errors were replaced by their neighboring average (IPL program ADESPIKE). To remove instrumental variations in sensitivity each pixel was divided by a white light flat field taken during the ground calibration through each filter. This correction for the relative radiometry was performed after the dark current, obtained near the time the Jupiter images were taken, was subtracted. The output DN was determined from a 10 point transfer curve based on the laboratory calibration (IPL program FICOR77).

The remaining reseaus were removed by replacing their pixel DN with interpolated values from neighboring pixels. The methane and green images were registered to the orange using the IPL interactive system and the PICREG program to determine common features and perform a cross correlation to establish reference coordinates (tie points). The correlation was calculated using $8 \times 8$ pixel areas centered about the reference tie points. The methane and green images were then mapped onto the orange image using GEOMA. The first two sets of images listed in Table I cover a significant fraction of the planet and these were mapped to a cylindrical projection coordinate system. The cylindrical projection mosaics contain 8-bit pixels, while the $10 n n \times 10 n n$ images contain 16-bit pixels.

Ouantitative estimates of the uncertainty in the data require knowledge of the signal shot noise and equivalent electron read noise, dark current values, uncertainty in the flat field calibration image, accuracy of the transfer curve for each pixel, the magnitude of the residual image and the recent exposure history of the vidicon, and temporal variability including the 
warmup of the instrument. The low gain state was set such that the rms read. noise would be 0.6 DN (private communication, Gary Bailey, 1984). Some inflight calibrations have been performed and we are assured (G. E. Danielson, and L. A. Soderbiom, private communication, 1984) that the long-time temporal stability of the narrow-angle cameras are of the order of $1 \%$, although shortterm stablity during warmup is significantly worse. The wide angle cameras have not been scrutinized as well as the narrow angle cameras. The working hypothesis among Voyager Imaging team members is that the relative (pixel-topixel, and picture-to-picture) accuracy is about $2 \%$ (for several pixels averaged together) over most of the frame for raw DN's near half-saturation (128), (G. E. Danielson and L. A. Soderblom, private communication, 1984). The sensitivity corrections for the corners and left and top edges of the frame are substantial and these areas are known to be of much poorer photometric quality.

We independently attempted to verify the stated accuracy by recalibrating a sequence of $1000 \times 1000$ pixel flat field images taken in the lab before launch with the wide angle camera. We processed the images with FICOR77 and found a a $4 \%$ gradient from the lower left line 800 , sample 200 to the upper right line 200, sample 800 ( 1 ine 1, sample 1 define the upper left corner) in a half-saturated image. The residuals are worse for images with lower or higher DN levels, especially in the corners and near the left and top edges at the higher ON levels as expected. At low DN levels digitization produces an uncertainty of several percent. Away from the edges there is very little power in high spatial frequencies in the calibrated lab images. The single pixel rms noise is about $1 \mathrm{DN}$ at a mean level of $128^{\circ} \mathrm{DN}$. Small scale absorption regions (5-10 pixels across) in the Jovian atmosphere should be easily distinguished if their average DN differs by 1 DN or more from 
neighboring pixels.

We use the figure of $\pm 2 \%$ uncertainty in the relative photometry based on our conversations with Imaging team members. However, we call attention to the fact that a comprehensive analysis of the photometric precision of the Voyager cameras using flight data has not been carried out. A study as thorough as Young's (1974) publication is required before estimates of the photometric precision can be taken with confidence.

The IPL program FICOR77 uses absolutely calibrated ground-based spectra of the Galilean satellites to assign absolutely calibrated I/F values for green and orange frames but not for methane frames. The uncertainty in the absolute calibration is thought to be about $\pm 10 \%$ (G.E. Danielson, private communication, 1984). The I/F values from the Voyager $1000 \times 100016-b i t$ images are several percent higher than the spectra of Cochran et al. (1981). We are unable to resolve the question of which to believe. We have chosen to use the Voyager calibration but we perform a sensitivity study to estimate how : strongly our results depend on the absolute calibration. The calibration factor given in the header records for our 8-bit cylindrical projection images (the first two sets in Table I) is incorrect by roughly an order of magnitude and we have applied our own calibration factor to those images.

We have calibrated the methane frames relative to the orange and green frames. We first found the relationship between the equivalent width of the methane absorption band and the methane/continuum ratio by generating synthetic spectra from equation (1) and integrating over the response functions of the green, orange and methane filters. The quantity $R_{C_{4}}=1$ $0.00546 W$ where $W$ is the 6190 \& equivalent width in \&. From our analysis of the spectra of Cochran et al. (1981), and Cochran and Cochran, (1980), we estimate $W$ for the NTrZ to be $16 \pm 2 \AA$. The measurements of Cochran and 
Cochran (1980) are for the EZ. In our images the EZ and NTrZ have nearly identical values of $\mathrm{R}_{\mathrm{CH}_{4}}$ when large regions are averaged. We next derived a correction for the phase angle dependence of $W$ based on the models of West and Tomasko (1980). At $25^{\circ}$ phase angle the calculated equivalent width is $17.6 \mathrm{~A}$, and $\mathrm{R}_{\mathrm{CH}_{4}}=0.904$. The average $\mathrm{R}_{\mathrm{CH}_{4}}$ in the $\mathrm{NT} r \mathrm{Z}$ in the images with FDS = 16378.04 - 16378.12 require that the reduced methane images from IPL be multiplied by the factor 1.36 to give the correct ratio.

The effects of planetary rotation during the 7 and 11 minute time intervals of the first two sets of images are noticeable at some distance from the central meridian. We made a first-order photometric correction to compensate for differences in lighting and viewing angles by fitting the orange NTrZ data to a Minnaert limb darkening law I/F $\mu=$ const $(\mu \mu O)^{k}$. We multiplied each pixel in the green and methane images by the factor $\mu / \mu_{0}$ ange

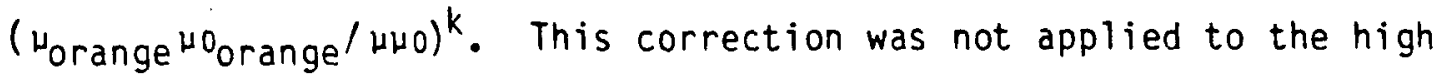
resolution images since $\mu \simeq \mu_{0} \simeq 1$ and $\Delta \mu$ and $\Delta \mu_{0}$ are small over the entire: image. A noticeable limb-to-terminator gradient remains in the cylindrical map ratio and spectral gradient images even after applying the correction. These effects and the uncertain calibration mentioned above limit our ability to interpret these images and most of the subsequent analysis is based on the high resolution images. 


\section{RESULTS}

The cylindrical projection maps appear in Figs. 2-4. These show the orange (I/FO), spectral gradient $\partial / \partial \lambda\left(I / F_{c}\right)$, and $R_{C_{4}}$ over a modest portion of the planet. The inability to achieve good image registration near the limb due to foreshortening is apparent and we ignore these areas. Subtle differences between belts and zones are evident in the spectral gradient map. The most striking feature is the dark 'barge' near $14^{\circ}$ latitude which has the strongest spectral gradient of any cloud on the planet. We will discuss this feature in more detail later.

The planet is remarkably uniform in the methane/continuum image. Some notable exceptions with low methane/continuum ratios are the region adjacent to the plume head at latitude $6^{\circ}$, longitude $90^{\circ}$, and the perimeter of the Great Red Spot. Both regions are close to or identical with 5 m hot spots and appear in the higher resolution images discussed in later.

Few systematic differences, if any, between belts and zones are apparent in the methane/continuum ratio image. This conclusion significantly strengthens ground-based results (Teifel, 1976, West, 1979a, Woodman et al., 1979) which were limited by their low spatial resolution. Figure 5 shows how the reflectivity and equivalent width vary with latitude near the central meridian, at system III longitude $255^{\circ}$. Figure 6 shows the relationship between $W$ and continuum reflectivity. The highest value of $W$ for .the longitude range $250^{\circ}-260^{\circ}$, latitudes $-18^{\circ}$ to $+33^{\circ}$ occurs in the NEB near $10^{\circ}$ latitude. From Fig. 6 we conclude that any correlation of $W$ and continuum $I / F$ is weak, and $W$ can vary between about 12 and $20 \mathrm{~A}$ in both belts and zones.

The large value of $W$ at $10^{\circ}$ latitude in Fig. 5 is near a local maximum in methane absorption in the NER. Figure 7 shows the longitudinal structure of $W$ in the Equatorial Zone when the image is degraded to ground-based resolution 
(convolved with a window $16^{\circ}$ in longitude by $11^{\circ}$ in latitude) to facilitate comparison with the data of Cochran and Cochran (1980 and 1983). There is an east-west gradient in $W$ in both sections of the mosaic which is probably a consequence of our inablilty to completely compensate for changes in lighting and viewing angle over the time interval of the green-orange-methane sequence. Superimposed on the gradient are fluctuations in w with an amplitude of about 2 A. Cochran and Cochran also observed fluctuations of about the same amplitude. Cochran and Cochran (1983) call attention to a local minimum in $W$ at $150^{\circ}$ system I longitude in their 1979 data and at $165^{\circ}$ in their 1980 data and a local maximum at $35^{\circ}$ system I longitude for both years. Our data show a local minimum near $150^{\circ}$ system I longitude, but $35^{\circ}$ longitude is between weak maximum and minumum values. The strongest relative maximum in our data occurs near $180^{\circ}$ system I longitude. The Cochran and Cochran data do not show a peak in methane ahsorption at the same longitude. We can see from Fig. 4 that small regions in Jupiter's NEB and around the GRS are more highly absorbing than the low resolution ground-based data reveal. These can be studied in even greater detail in the higher resolution images, shown in Figs. 8-19. We show the orange continuum image, the continuum spectral gradient, and the methane/continuum ratio.

Spectral gradients are small for all cloud features except the brown barge" near $14^{\circ}$ latitude in Fig. 9. The barges are distinguished both by their low continuum reflectivity and by their strong continuum gradient near 6000 A. This suggests real compositional or allotropic differences between barges and other cloud features. For example, allotropes of $P_{4}$ have strong gradients in the 6000 \& region but most, if not all, allotropes of elemental sulfur have flat spectra longward of 5000 \& (Fig. 20; J. Gradie, private communication, 1982). 
Sulfur ions in solution may have strong gradients near 6000 \& (Chivers, 1977; Salahub et al., 1978). What we are concerned with is the behavior of the single scattering albedo and optical depth of aerosols, not a surface deposit, as a function of wavelength. So it is premature to rule out sulfur as the active chromophore in barges. By the same reason, without consideration for other arguments relevant to chemical reaction rates and mass balance, it is difficult to rule out elemental or ionic forms of phosphorus as the coloring agent for the GRS. Although the laboratory measurements for $P_{4}$ in Fig. 20 are not consistent with the observed spectral gradient in the GRS, laboratory measurements on aerosols are needed to make an informed judgment. Discussions of candidate chromophores are not very informative so long as they rely on qualitative descriptions such as 'tawny, brown, red'.

The methane/continuum ratio image (Fig. 10) is almost featureless. The barge has very neariy the same ratio as the NTrZ and NEB. A narrow region of bright pixels along the south west edge of the barge is a result of registration errors, and the same may be true of the crescent of dark pixels along the east edge. Deviations of $\mathrm{R}_{\mathrm{CH}_{4}}$ about its mean value are comparable to the $2 \%$ uncertainty level and are not correlated with reflectivity. Local minimum and maximum in $\mathrm{R}_{\mathrm{CH}_{4}}$ appear near line 400 sample 550 , and line 570 sample 450, respectively. These features are broad (tens of pixels across) and deviate by about $2.4 \%$ from the mean. They are not apparently related to cloud morphology. They could be produced by a single faussian shaped low sensitivity spot in the camera at line 520 sample 468 with a FWHM of 60 pixels. We were ahle to remove those features by multiplying the green, orange, and methane images with a correction flat-field having a bump of the stated size, magnitude, and location. We looked for evidence of a reduced sensitivity region on the other sets of images but were unable to confirm 
it.

Morphologically related absorption features are present in plume and GRS images (Figs. 14, 15, 17, and 18). Reflectivity and $\mathrm{R}_{\mathrm{CH}_{4}}$ are correlated in the GRS image in the sense that photons have longer scattering paths in the darker regions. Qualitatively this relationship agrees with the picture derived from 5 um images (Terrile and Beebe, 1979). But the strongest absorptions are associated with the bright plume head (Fig. 12 line 664 sample $51 n$ ) and the perimeter of the GRS (Fig. 16 line 444 sample 628). The methane/continuum ratio is as $10 \mathrm{w}$ as 0.84 in those regions. The complicated vortex patterns which occupy the interface region between the NEB and the NTrZ harbor a few isolated small spots of strong methane absorption (in Fig. 14, line 422, sample 306, iine 408, sample 46n, line 470, sample 612). Small spots within the GRS are also highly absorbing, although $\mathrm{R}_{\mathrm{CH}_{4}}$ integrated over the GRS is slightly greater than the value 0.905 for the average NTrZ. On ground-based images (West, 1979a, and 1979b) the GRS is indistinguishable from the STrZ in the 6190 \& band but is $30 \%$ brighter than the STrZ in the strong $8900 \AA$ band. Intermediate values of $\mathrm{R}_{\mathrm{CH}_{4}}$ are found in parts of the $\mathrm{NEB}$ and in the broad region of low reflectivity surrounding the GRS. Selected spot values $(10 \times 10$ pixel averages) are listed in Table II.

Three measurements which might be useful in characterizing upwellingdownwelling motions are the methane/continuum ratio and 5 um brightness temperature which are sensitive to the presence of clouds, and the molecular hydrogen para fraction at 300 mbar which depends on vertical mixing on time scales of $10^{9}$ seconds (Conrath and Gierasch, 1984). Strong vertical mixing would establish the para fraction, $f_{p}$, at its 'normal' value of 0.25 , while no vertical mixing would allow equilibration of $H_{2}$ with $f_{p}$ about 0.35 at the 300 mbar level. 
Contour maps of 5 micron brightness temperature (integrated over a 50 $\mathrm{cm}^{-1}$ window from 2000 to $2050 \mathrm{~cm}^{-1}$ ) and $f_{p}$ were generated from raster scan maps by the Voyager IRIS instrument. The reduced data were provided by $B$. Conrath (private communication, 1984). The location of the maps on the orange frames was established from support imaging of narrow-angle frames. These maps and high resolution ( $6 \times 6$ pixel bins) contour maps of $W$ are shown in Figs. $21-26$.

The highest 5 um brightness temperatures are found in low reflectivity regions of the NEB and hot spots (they are not physically hotter than their surroundings) and are confined to those regions in the Voyager maps. The IRIS signal/noise ratio at $5 \mathrm{~m}$ is poor for brightness temperatures below about 200 K. The brightness temperature $255 \mathrm{~K}$ corresponds to the 4 bar level in the atmosphere. The methane/continuum ratio in these regions is almost the same as in the NTrZ. This observation leads to the conclusion that we are not seeing down to 4 bars in the visible in hot spot regions, that substantial cloud material is present well above 4 bars in these regions, and that cloud stratigraphy based on 5 m data alone is erroneous. These conclusions will be supported quantitatively.

The $\mathrm{H}_{2}$ para fraction does not correlate with cloud reflectivity, 5 um brightness temperature, (lack of correlation of these parameters on the much larger scale of belt/zone structure was noted by Conrath and Gierasch) or methane/continuum ratio. A lack of correlation with cloud reflectivity does not imply that clouds are ineffective in catalyzing ortho-para conversion; cloud reflectivity is sensitive to the aerosol single scattering albedo, not the amount of aerosol. From Pioneer polarimetry (Smith and Tomasko, 1984) and ground-hased measurements in methane bands (West, 1979a, Woodman, 1979) strengthened by the present results, we know that substantial visible optical 
depths of aerosol are present in the $600-320$ mbar region in both low and high reflectivity regions.

We are now ready to discuss what our measurements of $\mathrm{R}_{\mathrm{CH}_{4}}$ in Tabie II and IRIS 5 um brightness temperatures imply for quantitative aerosol vertical structure models. The doubling-adding method (see Hansen and Travis, 1974) we use to calculate multiple scattering restricts our analysis to plane-parallel geometry (Diner and Martonchik, 1984 have generalized the doubling-adding method to horizontally inhomogeneous cases). Several structures have been suggested from.published studies of absorption bands and polarimetry (e.g. Sato and Hansen, 1979; Cochran and Cochran, 1980; West and Tomasko, 1980; Owen and Terrile, 1981; Smith and Tomasko, 1984; Stoker, 1984). One difficulty is to choose an appropriate structure and to explore a comprehensive yet manageable number of model parameters, and fix the remaining parameters based on solid observational constraints from the literature.

The weak 6190 A absorption is insensitive to the small amounts of aerosol in the upper 320 mbar so we fix the optical depth of aerosol above 320 mbar from Pioneer photometry at high phase angle (Tomasko et al., 1978), groundbased observations of reflectivity in the strong 8900 \& methane band (West, 1979a; West and Tomasko, 1980; Stoker, 1984), and Pioneer polarimetry (Smith and Tomasko, 1984). Our models have a thin (optical depth 0.125) forwardscattering haze layer at 80 mbar whose phase function is a Henyey-Greenstein function, $p(\theta, g)=\left(1-g^{2}\right) /\left(1+g^{2}-2 g \cos \theta\right)^{3 / 2}$. The asymmetry parameter, $g$ $=0.75$, and single-scattering albedo $(0.95)$ were determined from the observed high reflectivity at $150^{\circ}$ phase angle (Tomasko et al.. 1978). A second haze layer at 250 mbar provides a source of scattering to account for the high, and longitudinally uniform, reflectivity in the Equatorial Zone in the $8900 \AA$ methane band (West and Tomasko, 1980; Stoker, 1984). The altitude and optical 
thickness of this layer is not well constrained, and analysis of high phase angle polarization at equatorial latitudes would be helpful. Based on the 8900 \& reflectivity we choose $\tau=1.0$ for the $E Z$ at $0^{\circ}$ latitude, and $\tau=0.1$ for the NEB at $10^{\circ}$ latitude. Aerosols in this and all deeper layers have twoterm Henyey-Greenstein phase functions derived from Pioneer photometry (Tomasko et al., 1978) with $g_{1}=0.8, g_{2}=-0.7, f\left(\right.$ fraction with $\left.g_{1}\right)=$ 0.938 .

The ammonia cloud top appears to be at a constant level ( 320 mbar) in both belts and zones (Smith and Tomasko, 1984) and appears to be Tongitudinally uniform from the weak longitudinal variahility of reflectivity at $8900 \AA$ (West and Tomasko, 1980; Stoker, 1984). The ammonia condensation level is near 700 mbar. Our two free parameters are then the optical thickness in the $\mathrm{NH}_{3}$ cloud, $\tau_{\mathrm{NH}_{3}}$, uniformly mixed with the gas between 700 and $320 \mathrm{mbar}$, and the pressure at the top of a lower cloud, $\mathrm{p}$, whose composition is not specified but is likely to be $\mathrm{H}_{2} \mathrm{O}, \mathrm{H}_{2} \mathrm{O}-\mathrm{NH}_{3}$ solution, or $\mathrm{NH}_{4} \mathrm{SH}$. We assume the lower cloud is dense enough to prevent penetration of photons into the cloud so we ignore absorption within or below the lower cloud to limit the number of free parameters. The continuum single scattering albedo is independent of height at $6190 \AA$. In the 5 un region the lower cloud top is treated as a pure absorbing/emitting surface with emissivity $=1$. The structure is illustrated in Fig. 27.

Methane absorption

To relate pressure to methane column abundance we assume a $\left[\mathrm{CH}_{4}\right] /\left[\mathrm{H}_{2}\right]$ volume mixing ratio of $2 \times 10^{-3}$ (Gautier et al., 1982), and $[\mathrm{He}] /\left[\mathrm{H}_{2}+\mathrm{He}\right]=$ 0.1. The pseudo-continuum methane absorption coefficients from Giver (1978) were averaged over the bandpasses of the green, orange and methane filters to 
give filter averaged absorption coefficients for use in the multiple scattering calculations. The use of filter averaged coefficients is more efficient than a calculation every 10 \& and subsequent integration over the band. We checked the results with a 10 \& resolution calculation to verify the accuracy of the approach. The average absorption coefficients are $\langle k\rangle=$ $0.037,0.075$, and 0.362 for the green, orange, and methane filters, respectively. In the model study we calculated I/F for the continuum single scattering albedo at $6190 \AA$ for $\langle k\rangle=0.075$ and for $\langle k\rangle=0.362$ and took the ratio $I / F(\langle K\rangle=0.362) / I / F(\langle K\rangle=0.075)$ for $R_{C_{4}}$. The temperature dependence of the 6190 \& band is not well know but is thought to be small (Ramaprasad et a1., 1978). Laboratory measurements of the temperature dependence of the near-infrared and visible methane bands are urgently needed.

Figures 28-31- show how $\mathrm{R}_{\mathrm{CH}_{4}}$ depends on ${ }^{\top} \mathrm{NH}_{3}, P$, cloud single scattering albedo (w) and the optical depth of the haze at $250 \mathrm{mbar}$ (Thaze). These results were obtained for a scattering geometry appropriate for the plume head (entry 13 in Table II) for Figs. 28-31, and for the Barge (entry 1 in Table II) for $\omega=0.97$. The cases $\omega=0.998,0.991$ and 0.970 give the observed $I / F_{C}$ in the plume head and NTrZ, NEB, and barge, respectively. The scattering geometry for the NTrZ (entries 27-32 in Table II) have $\mu$ and $\mu$ o similar enough to the plume that Figs. 28 and 29 can be applied to those regions as well.

The radiative transfer solution for each observed value of $\mathrm{R}_{\mathrm{CH}_{4}}$ maps out a curve in $\tau-P$ space. Neither $\tau$ nor $P$ are separately well determined but the T-P curve is well defined by $\mathrm{R}_{\mathrm{CH}_{4}}$. It is this aspect of the non-unique solution to this kind of inverse problem that has led to many different models in the literature. Since hoth weak $\mathrm{H}_{2}$ lines and weak $\mathrm{CH}_{4}$ bands follow similar curves in parameter space the multiplicity of solutions can hardly be narrowed 
by fitting both, but the $\mathrm{CH}_{4} / \mathrm{H}_{2}$ mixing ratio can therefore, in principal, be determined with.little sensitivity to the model parameters unless the model is simply inadequate (e.g. a reflecting layer model). The Sato and Hansen (1979) models correspond to the large- $\tau$, large-P end of the curve while the models of West (1979a) are at the opposite end of the curve, although there are other differences as well. Additional constraints are needed to refine the analysis. For example, the reflectivity and center-to-limb variations in the 8900 \& band observed at ground-based resolution (West, 1979a) restrict ${ }^{{ }^{2} \mathrm{NH}_{3}}$ to the range $3-5$, and $P=1.5$ bar in the NTrZ. If observations at Voyager resolution were available and allowed us to apply the constraint $3<\tau_{\mathrm{NH}_{3}}<5$ in the plume head as well, the variation in $\mathrm{R}_{\mathrm{CH}_{4}}$ must be due primarily to variations in the lower cloud boundary, between 1.5 and 3 bar.

The sensitivity of $\mathrm{R}_{\mathrm{CH}_{4}}$ to the absolute calibration in the continuum, and hence to us can be judged from Figs. 29-31. As w decreases $\mathrm{R}_{\mathrm{CH}_{4}}$ increases because the multiple scattering path length decreases. The $\mathrm{NH}_{3}$ cloud must be optically thinner and/or the bottom cloud deeper in the barge to keep ${ }^{R} \mathrm{CH}_{4}$ nearly the same as in the NTrZ. If the $\mathrm{NH}_{3}$ optical depth and $\mathrm{P}$ in the NTrZ are 3.0 and 1.5 bar, respectively, and if $\tau_{\mathrm{NH}_{3}}$ is the same in the barge, the lower cloud top must be near $P=3$ bar in the barge.

We can dismiss the notion that at visible wavelengths we are seeing through a thin aerosol layer to the 4 bar region in hot spots, at least for the two cases observed here. The average value of $\mathrm{R}_{\mathrm{CH}_{4}}$ in hot spot regions is 0.890. Figure 30 shows that if the lower cloud top is at 4.5 bar, the sum of $\mathrm{NH}_{3}$ cloud plus haze optical depth is 7 at $6190 \mathrm{~A}$. If the ammonia cloud were absent the methane/continuum ratio would be 0.78 and the equivalent width would be $40 \AA$. The smallest value $\mathrm{R}_{\mathrm{CH}_{4}}$ in our images is 0.84 , and this value is found in the bright plume head, at locations along the perimeter of the 

curve with $\mathrm{R}_{\mathrm{CH}_{4}}=0.92$ (corresponding to $\mathrm{W}=14.5 \mathrm{~A}$ ) calculated for $10^{\circ}$ phase. angle (not shown) are nearly coincidental, justifying our earlier statement that weak methane and $\mathrm{H}_{2}$ lines should be highly correlated in the volume of parameter space which fits the data. The mixing ratio $\left[\mathrm{CH}_{4}\right] /\left[\mathrm{H}_{2}\right]=2.2 \times 10^{-3}$ provides the best fit to the adopted ratio of $W\left(\mathrm{H}_{2}\right) / W\left(\mathrm{CH}_{4}\right)$. For normal $\mathrm{H}_{2}$ an $8 \mathrm{~m} \AA$ equivalent width corresponds to a slightly higher value $(0.925)$ of $\mathrm{R}_{\mathrm{CH}_{4}} \cdot$

Hunt and Bergstralh report $\mathrm{H}_{2}$ 4-0 equivalent width between 4.5 and 12.5 $m \AA$ which they interpret as real variability in the atmosphere. These extremes would imply variations in the 6190 \& methane equivalent width of $8.6-24 \AA$, much larger than observed by Cochran and Cochran (1980; 1983) and larger than seen in the Voyager data when degraded to ground-based resolution. It seems likely that some of the variability reported by Hunt and Bergstralh is not intrinsic to Jupiter. Carleton and Traub (1974) remark on the constancy of the 4-0 S(1) line and the variability of the 3-0 S(1) line.

Carleton and Traub measured 3-0 S(1) equivalent width ranging from 34 to $50 \mathrm{~mA}$. Hunt and Bergstralh report values ranging from 35 to $63 \mathrm{~mA}$. Variations of the magnitude reported are difficult to understand in light of the much weaker variability, at ground-based resolution, of the methane and 40 equivalent width, except for the 4-0 variability reported by Hunt and Bergstralh. Comparison of Figs. 32 and 33 shows that the $3 \div 0 \cdot s(1)$ line could vary between 52 and $42 \mathrm{~m} \AA$ while the 4-0 line equivalent width remained constant if $\tau$ and $P$ varied in a contrived way precisely along the 4-0 $8 \mathrm{~m} \AA$ trajectory between the low $\tau$ - low $P$ end to the high $\tau$ - high $P$ end. The equivalent widths of the 3-0 and 4-0 lines are not as well correlated as those of the 4-0 $\mathrm{H}_{2}$ and 6190 \& methane band because the absorption coefficient at line center in the 3-0 band is much stronger than absorption coefficients for 
the other line and band. Some variability between high and low reflectivity regions is expected from differences in photon scattering path lengths as discussed ahove for $\mathrm{CH}_{4}$ absorption, although the observed $\mathrm{CH}_{4}$ equivalent width is not correlated with reflectivity (Fig. 6).

A critical test of the quality of the $\mathrm{H}_{2}$ observations is the 3-0 $S(1) / S(0)$ ratio. The rotational $S(1)$ and $S(0)$ pressure induced dipole lines used by Conrath and Gierasch (1984) to estimate the para fraction were found to lie within the extremes corresponding to equilibrium and normal hydrogen in the Equatorial Zone. The calculated equivalent width ratios near the center of the disk for equilibrium and normal hydrogen are shown in Figs. 34 and 35. The ratio is near 1.5 over most of the parameter space for equilibrium hydrogen and is somewhat higher for normal hydrogen, ranging between 1.65 and 1.85. The measurements of Hunt and Bergstralh give ratios between 1.2 and 1.8, with a cluster of values near 1.5. If we discard measurements with low and high values of $3-n S(1) / S(0)$ (near 1.2 and near 1.8), and measurements of the $S(1)$ line when no simultaneous measurements of $S(0)$ exist, the remaining measurements of the $S(1)$ equivalent width cluster between 51 and $63 \mathrm{~m} A$. These values are systematically higher than those reported by Carleton and Traub. If they are to be consistent with the 4-0 $S(1)$ measurements, including those of Carleton and Traub, and the 6190 \& methane equivalent width measurements, the $\mathrm{NH}_{3}$ optical depth should be in the range $0-6$, and $P$ should be in the range 1.3 - 1.8 bar. The apparent large variability, and the low values of the 3-0 S(1) equivalent width reported by both Carleton and Traub and Hunt and Bergstralh are nevertheless troubling and need to he explained. A major contribution to uncertainty in the models is the wide range of observed values found in the literature. 


\section{Thermal emission at $5 \mathrm{Lm}$}

Calculations at 5 um include multiple scattering using the doubling and adding technique. The adding equations discussed by Hansen and Travis (1974) are easily generalized to include thermal radiation. The emissivity of a Plane-parallel layer of optical depth $\tau$ whose azimuth-independent components of diffuse reflection and transmission (Chandrasekhar (1960)) are $S\left(\mu, \mu_{0}\right)$ and $T(\mu, \mu 0)$, is given by

$$
\varepsilon(\mu)=1-e^{-\tau / \mu}-\frac{1}{2 \mu} \int_{0}^{1} S\left(\mu, \mu^{\prime}\right)+T\left(\mu, \mu^{\prime}\right) d \mu^{\prime}
$$

Multiple bounce terms at the interface of two layers are calculated in the same way as for reflected sunlight. The atmosphere is "added up" starting at the bottom, and the outging radiance and cumulative $S$ function of the lower layers are retained at each step. Only the azimuthally independent component needs to be calculated.

Building on the work of Kunde et al. (1982), Bjoraker (1984) has computed absorption coefficients in the $5 \mathrm{~m}$ region for 8 gases $\left(\mathrm{H}_{2}\right.$, broadened by $\mathrm{H}_{2}$ and $\mathrm{He}, \mathrm{NH}_{3}, \mathrm{PH}_{3}, \mathrm{H}_{2} \mathrm{O}, \mathrm{CH}_{3} \mathrm{D}, \mathrm{CH}_{4}, \mathrm{GeH}_{4}$, and $\left.\mathrm{CO}\right)$. He kindly sent us a copy of his opacity tapes. These tapes incorporate recent laboratory measurements and Bjoraker describes several additional improvements over those used by Kunde et al.. Coefficients are tabulated on a 35-level pressure-temperature grid between 12 bar and 0.08 mbar, shown in Fig. 27, at a mean frequency. interval of about $0.02 \mathrm{~cm}^{-1}$. The resolution of the IRIS instrument is $4.5 \mathrm{~cm}^{-1}$. We integrate the radiance from 2000 to $2050 \mathrm{~cm}^{-1}$ to compare with the maps in Figs. 22 and 25. To determine the effects of aerosols on the integrated radiation field one does not need the high spectral resolution provided on the 
tapes. We instead use the Chandrasekhar mean, or flux weighted mean absorption coefficient calculated for each layer at intervals of $2 \mathrm{~cm}^{-1}$, from a full resolution synthetic spectrum without aerosols. Gas abundance profiles were taken from Kunde et a1. (1982). It should be kept in mind that the pressure broadened far-wing profile is not well known, and the Lorentz profile was truncated at $50 \mathrm{~cm}^{-1}$ from line center. The far-wing line shape contributes a major uncertainty in determining aerosol content in hot spot regions.

Figure 36 . shows the line-by-line synthetic spectrum, and the $2 \mathrm{~cm}^{-1}$ Chandrasekhar mean coefficient spectrum with and without aerosols. The $2 \mathrm{~cm}^{-1}$ averaged spectrum is indistiguishable from the unaveraged spectrum except at the steep gradient at $1875 \mathrm{~cm}^{-1}$. We keep the abundance and temperature profiles fixed and vary only the aerosol optical depth in the $\mathrm{NH}_{3}$ region and the lower boundary pressure level. The dotted curve in Fig. 36 is produced by a model with a black-body surface at 6.08 bar, and an $\mathrm{NH}_{3}$ cloud with constant optical depth (1.5) and single scattering albedo (0.865) throughout the 1800 $2200 \mathrm{~cm}^{-1}$ range.

The optical properties of Jovian aerosols in the 5 um region are uncertain. We use the optical constants of $\mathrm{NH}_{3}$ ice at $2025 \mathrm{~cm}^{-1}$ tabulated by Martonchik et al. (1984) and a Mie code to calculate the single scattering albedo and phase function (for the case $r_{\text {eff }}=1$ um only). Mie calculations provide only an approximate description of the phase function and single scattering albedo for nonspherical crystals and the results shown helow are therefore crude. Laboratory measurements and theoretical studies of the phase function of $\mathrm{NH}_{3}$ ice crystals in the 5 um region are needed. Orton et al. (1982) tried several candidate phase functions, including one derived from an extrapolation of laboratory measurements in the visible (Holmes, 1981). Both 
Marten et al. (1981) and Orton et al. favor particles large compared to the wavelength from their analyses of thermal emission. We do not feel constrained by those studies because they were not directed specifically at hot spot regions. For the present we show results for large particles, (assuming the extinction optica) depth and phase function are the same in the visible and at $5 \mathrm{~mm}$ ) and particles with effective radii of $1 \mathrm{~mm}$, whose extinction efficiency is a factor of 5 smaller at 5 un than it is at $6190 \AA$. The 1 m particles have single scattering albedo 0.971 and their phase function is similar to a Henyey-Greenstein function with asymmetry parameter 0.5. The large particles $\left(r_{\text {eff }}=30 \mathrm{~mm}\right)$ have $\omega=0.865$ at $5 \mathrm{~mm}$. Their phase function is defined to be the same two-term Henyey-Greenstein function used for the visible wavelength models.

In the absence of aerosols the calculated $5 \mathrm{~m}$ brightness temperature is $275 \mathrm{~K}$ for nadir viewing. The cloud top at 6 bar or higher restricts the brightness temperature to be $270 \mathrm{~K}$ or less. The $\mathrm{NH}_{3}$ cloud and haze layers further diminish the emerging radiance, such that the high brightness temperatures observed $(255 \mathrm{~K})$ in the NEB imply ${ }^{{ }^{\top}} \mathrm{NH}_{3}<1.5$ and $\mathrm{P}>4.5$ bar if the particles have the same optical depth and phase function at visihle wavelengths and at $5 \mathrm{~lm}$ as in Fig. 37. But Fig. 30 indicates that $\mathrm{R}_{\mathrm{CH}_{4}}$ for the region of parameter space defined above is less than 0.8 , much lower than the observations show.

Figures 30 and 38 show that both observational constraints $\mathrm{R}_{\mathrm{CH}_{4}}=0.89$ and $T_{5}$ um $=255 \mathrm{~K}$ can marginally he satisfied if the particles in the $\mathrm{NH}_{3}$ cloud have mean radi $i$ of $1 \mathrm{~mm}$, optical depth $7-8$ at $6190 \AA$, and $P=6$ bar. If the particles were even smaller than 1 un the stronger wavelength dependence of their extinction coefficient would ease the difficulty of fitting visible and 5 im data, but arguments advanced by Marten et al. (1981) and Orton et al. 
(1982) advocating larger particles would have to be answered. As mentioned earlier, those arguments were motivated by observations of broad belt and zone regions, not hot spots. 


\section{CONCLUSIONS}

We have succeeded in answering a number of questions posed in the introductory section of this paper, but a number of issues are still unsettled. The forlowing paragraphs summarize the conclusions, describe the outstanding problems, and suggest laboratory measurements and observations which would help resolve them.

We have successfully used three of the voyager 1 filters to formulate quantitative measures of the red continuum reflectivity, spectral gradient, and methane/continuum ratio, or alternatively, the 6190 \& band equivalent width. Most features have a flat or negative spectral gradient near $6000 \AA$, but the dark barges have strong positive gradients, suggesting allotropes or compounds not present in other regions. Comparison with a few laboratory. frost spectra argue against elemental sulfur as the coloring agent in barges, but laboratory measurements of candidate aerosols, not frosts, are needed before a definitive statement can be made.

Over large regions, at a scale corresponding to earth-based resolution, the methane/continuum ratio shows small variations, on the order of $10 \%$ ahout the mean value. At higher spatial resolution, small regions can have significantly greater methane absorption, but not as much as an atmosphere which is essentially clear down to the 5 bar level. The barges have the same methane/continuum ratio as the NTrZ. This fact, when coupled with a multiple scattering calculation which takes into account their low continuum single scattering albedo, implies visible photons penetrate to greater depths in these regions than in the NTrZ. If the $\mathrm{NH}_{3}$ cloud optical depth at $6190 \AA$ is 3-5 in both barges and the NTrZ, a dense cloud at 1.5 bar in the zone, and 3-5 bar in the barge produces the observed methane/continuum ratio. This structure is but one of a continuum of possible structures which lie along the 
solution curve in parameter space.

The cloud parameterization chosen here has two haze layers, one at 80 mbar and one at 250 mhar, an $\mathrm{NH}_{3}$ cloud with chromophores between the 320 and 700 mbar level, and a deeper dense cloud, with the same single scattering albedo as the $\mathrm{NH}_{3}$ cloud. The structure chosen for this study is based on observational constraints from ground-based methane band studies and photometry and polarimetry from Pioneers 10 and 11 . Some modification to the structure (e.g. relaxing the requirement that the single scattering albedo be the same in the lower and upper cloud) is possible without violating the observational data, and regions such as hot spots, which have not been scrutinized by previous investigators or which are unresolved from the ground are not truely constrained by previous studies. For the models used in this study, a curve in the two free parameters fits the data. The observed quantity can be produced by a shallow atmosphere with a thin $\mathrm{NH}_{3}$ cloud, or by a deep atmosphere with a thick cloud.

Model studies indicate that the weak $6190 \AA$ band and the $\mathrm{H}_{2}$ 4-n. S(1) line should be highly correlated, and that a methane/hydrogen mixing ratio based on the observed equivalent width ratio should be insensitive to the model. The stronger 3-0 S(1) line is not as well correlated with the $6190 \AA$ band, but should display ahout the same variability. The reported variability for the 3-0 S(1) line.(Carleton and Traub, 1974; Hunt and Bergstralh, 1977) is much greater than the variability of the 6190 \& band at ground-based resolution, and is as difficult to understand today as it was when Carleton and Traub first remarked on it. Further study of the 3-0 lines is needed.

This is the first investigation to combine simultaneous observations of methane absorption at visible wavelengths and thermal emission at 5 um. Since both observations are sensitive to cloud opacity in the same altitude range, 
one might expect them to be highly correlated. In some instances, this is observed to be the case, especially in the region of the Great Red Spot. One of the surprises of this study is that, in both hot spots observed by IRIS with brightness temperatures reaching $255 \mathrm{~K}$, the observed methane/continuum ratio is almost the same as it is in the NTrZ, and is much higher than for an atmosphere which is essentially cloud-free down to 5 bar. Another surprise is that the bright plume head just south of the hot spot is a region of strong methane absorption. The idea that plume heads are upwelling centers of convective activity (Hunt et al., 1981; Stoker, 1983) may be viable if the associated downwelling regions are also in the plume head, instead of the surrounding area as previously suggested.

Direct comparison of methane absorption and 5 um emission brings into question our ideas about diagnostics for upwelling or downwelling. Both the methane absorption and $5 \mathrm{~mm}$ emission are related to cloud opacity, but they are apparently telling different stories in some important instances. We have made a first attempt to fit both observations quantitatively for hot spot regions. We calculated radiative transfer in clouds in a realistic way by including multiple scattering by aerosols for two cases - large particles whose phase function and extinction optical depth are the same at $5 \mu \mathrm{m}$ and in the visible, and smaller particles ( 1 um mean radius) whose optical depth is a factor of 5 smaller at 5 im than at 6190 \&. Although diffuse transmission through the cloud at 5 um permits a significant fraction of the upwelling radiation to penetrate the cloud, the upper limit to the $5 \mu \mathrm{m}$ optical depth in the $\mathrm{NH}_{3}$ cloud in hot spot regions is about 1.5. If optical depth is independent of wavelength between the visible and 5 um this cloud structure would allow significant penetration of visible photons to the 6 bar level, and the methane/continuum ratio would be much lower than observed. 
A larger optical depth is allower if the cloud single scattering albedo is higher than 0.865 at $5 \mathrm{~mm}$, or if the phase function were more forward scattering than the two-term Henyey-Greenstein function used here. Laboratory measurements of $\mathrm{NH}_{3}$ and candidate chromophore aerosol scattering properties at $5 \mathrm{um}$ are needed. Laboratory measurements of the temperature dependence of the the visible and near-infrared methane bands are also badly needed. An additional uncertainty which should be addressed is the far wing line absorption profile. Barring the possibilities that the aerosol is significantly more forward scattering and more nearly conservative than we assumed here, we are led to the conclusion that in hot spots, the aerosols in the $\mathrm{NH}_{3}$ cloud region have mean radii near 1 im or less, and their optical depth is about 7-8 in the visible and 1.5 at $5 \mathrm{~mm}$. It is conceivable that different structures, perhaps with aerosols in the 2 bar region rather than in the $\mathrm{NH}_{3}$ cloud region could also fit the data, but their total optical depth down to 6 bar should be roughly constant.

Other independent observations would further constrain the atmospheric structure. In particular, the CTLV behaviors of the absorption and blue reflectivity are good diagnostics of the optical depth in the $\mathrm{NH}_{3} \mathrm{cloud}$ and the altitude of chromophores. The Pioneer photometry and polarimetry, Voyager images, and thermal spectra have not been fully exploited. Other kinds of data would also help define the cloud structure. The Wide Field/Planetary camera on Space Telescope will be able to image Jupiter with resolution similar to the Voyager cameras at wavelengths from the UV to 1 um, including a methane filter at 8900 A. Instruments designed for the Galileo spacecraft are especially well suited to this broad multidisciplinary problem. Imaging, spectroscopy, radiometry, and polarimetry from the UV to $5 \mathrm{um}$, and in several methane bands, will provide a wealth of valuable information on cloud 
structure.

\section{ACKNOWLEDGEMENTS}

We have benefitted frombiscussions with R. Beebe, G. Bjoraker, B. Conrath, G. E. Danielson, J. Gradie, V. Kunde, G. Orton, L. Soderblom, and A. Young. We are especially grateful to G. Bjoraker who supplied molecular absorption coefficients for the $5 \mathrm{um}$ region in advance of publication of his Ph.D. dissertation. J. Gradie provided spectral reflectance data for solid S and $\mathrm{P}_{4}, \mathrm{~B}$. Conrath provided tables of 5 in brightness temperature and $\mathrm{H}_{2}$ para fraction. F. Herbert gave us a copy of his Galatry line shape code. A. Bahrami and W. Iwata helped with the computing. E. Corsmo and $f_{\text {. }}$ Garneau at IPL assisted with image processing. This research was sponsored by the NASA Jupiter Data Analysis Program, the Planetary Atmospheres Radiative Transfer program, and the Voyager project. 


\section{REFERE:NCES}

Bjoraker, f. (1984). The gas composition and vertical cloud structure of Jupiter derived from spectroscopic observations at $5 \mathrm{~mm}$. Ph.D. Dissertation, University of Arizona.

Bragg, S. L. (1981). An experimental study of the vibrational-rotational spectrum of molecular hydrogen, Ph.D. Dissertation, Washington University. Carleton, N. and W. Traub (1974). Observations of spatial and temporal variations of the Jovian $\mathrm{H}_{2}$ quadrupole lines. in Exploration of the Planetary System (A. Wosczcyk and C. Iwaniszewska, Eds.), pp. 351-356. Reidel, Dordrecht.

Castleman, K.R. (1979). Digital Image Processing, Prentice-Hall, Inc., New Jersey.

Chandrasekhar, S. (1960). Radiative Transfer. Dover, New York.

Chivers, T. (1977). in Polychalcogenide anions in Homoatomic Rings, Chains and Macromolecules of Main-Group Elements, Proc. of the IX Hudson Symposium. (A. L. Rheingold, Ed.), Pp. 499-537. Elsevier, New York. Cochran, A. L., L. M. Trafton, W. D. Cochran, and E. S. Barker (1981). Spectrometry of Jupiter at selected locations on the disk during the 1979 apparition. Astron. J. 86, 1101-1107.

Cochran, W. D., and A. L. Cochran (1980). Longitudinal variability of methane and ammonia bands on Jupiter. Icarus 42, 102-110.

Cochran, W. D., and A. L. Cochran (1983). Longitudinal variability of methane and ammonia bands on Jupiter II. Temporal variations. Icarus 56, 116-121. Cochran, W. D., J. Gelfand, and W. H. Smith (1976). Spatially resolved spectroscopy of Jupiter. I. $\mathrm{H}_{2}$ 4-0 S(1) line. Astrophys. J. 207, 639-645. 
Conrath, B. J., and P. J. Gierasch (1984). Global variation of the para hydrogen fraction in Jupiter's atmosphere and implications for dymanics on the outer planets. Icarus 57, 184-204.

Danielson, G. E., P. N. Kupferman, T. V. Johnson, and L. A. Soderblom (1981). Radiometric performance of the Voyager cameras. J. Geophys. Rev. 86, 8683-8689.

Diner, D. J., and J. V. Martonchik (1984). Atmospheric transfer of radiation above an inhomogeneous non-Lambertian reflective ground I. Theory. $\mathrm{J}$. Quant. Spectrosc. Radiat. Transfer in press.

Fink, I., and M. J. Belton (1969). Collision narrowed curves of growth of $\mathrm{H}_{2}$. J. Atmos. Sci. 26, 952-962.

Galatry, L. (1961). Simultaneous effect of Doppler and foreign gas broadening on spectral lines. Phys. Rev., 122, 1218-1223.

Gautier, D., B. Bezard, A. Marten, J. P. Baluteau, N. Scott, A. Cheddin, V. Kunde, and R. Hanel (1982). The $\mathrm{C} / \mathrm{H}$ ratio in Jupiter from the Voyager infrared investigation. Astrophys. J. 257, 901-912.

Giver, L. P. (1978). Intensity measurements of the $\mathrm{CH}_{4}$ bands in the region 4350 \& to 10500 \&. J. Quant. Spectr. Rad. Transfer 19, 311-322.

Hansen, J. E. and L. D. Travis (1974). Light scattering in planetary atmospheres. Space Sci. Rev. 16, 527-610.

Herbert, F. (1974). Spectrum line profiles: A generalized Voigt function including collisional narrowing. J. Quant. Spectrosc. Radiat. Transfer 14, 943-951.

Hess, S. L. (1953). Variations in atmospheric absorption over the disks of Jupiter and Saturn. Astrophys. J. 118, 151-160.

Holmes, A. W. (1981). Light Scattering from Ammonia and Water Crystals. Unpublished Ph.D. thesis, Univ. of Arizona. 
Hunt, G. E. (1973). Formation of spectral lines in planetary atmospheres. IV. Theoretical evidence for structure of the Jovian clouds from spectroscopic observations of methane and hydrogen quadrupole lines. Icarus 18, 637648.

Hunt, G. E., and J. T. Bergstralh (1977). Interpretation of spatial and temporal variations of hydrogen quadrupole absorptions in the Jovian atmosphere observed during the 1972 apparition. Icarus 30, 511-530. Hunt, G. E., B. J. Conrath, and J. A. Pirraglia (1981). Visible and infrared observations of Jovian plumes during the Voyager encounter. J Genphys. Res. $86,8777-8781$.

Kunde, V., R. Hanel, W. Maguire, D. Gautier, J. P. Baluteau, A. Marten, A. Chedin, N. Husson, and N. Scott (1982). The tropospheric gas composition of Jupiter's North Equatorial Belt $\left(\mathrm{NH}_{3}, \mathrm{PH}_{3}, \mathrm{CH}_{3} \mathrm{D}, \mathrm{GeH}_{4}, \mathrm{H}_{2} \mathrm{O}\right)$ and the Jovian D/H isotopic ratio. Astrophys. J. 263, 443-467.

Lutz, B. L., T. Owen, and R. D. Cess (1982). Laboratory band strengths of methane and their application to the atmospheres of Jupiter, Saturn, Uranus, Neptune, and Titan. II. The red region 6000-7600 A. Astrophys. J. 258, $886-898$.

Marten, A., D. Rouan, J. P. Baluteau, D. Gautier, B. J. Conrath, R. A. Hanel, V. Kunde, R. Samuelson, A. Chedin, and N. Scott (1981). Study of the ammonia ice cloud layer in the equatorial region of Jupiter from the Infrared Interferometer experiment on Voyager. Icarus 46, 233-248. Martonchik, J. V., G. S. Orton, and J. F. Appleby (1984). Optical properties of $\mathrm{NH}_{3}$ ice from the far infrared to the near ultraviolet. Applied Optics 23, $541-547$.

Mckeller, A. R. W. (1974). The significance of pressure shifts for the interpretation of $\mathrm{H}_{2}$ quadrupole lines in planetary spectra. Icarus 22, 
212-219.

Michalsky, J. J., R. A. Stokes, R. W. Avery, and W. C. DeMarcus (1974). Molecular band variations as a probe of the vertical structure of the Jovian atmosphere. Icarus 21, 55-65.

Orton, G. S., J. F. Appleby, and J. V. Martonchik (1982). The effect of ammonia ice on the outgoing thermal radiance from the atmosphere of Jupiter. Icarus 52, 81-93.

Owen, T. (1969). The spectra of Jupiter and Saturn in the photographic infrared. Itarus 10, 355-364.

Dwen, T., and H. P. Mason (1968). The abundance of hydrogen in the atmosphere of Jupiter. Astrophys J. 143, 317-326.

Owen, T. and R. J. Terrile (1981). Colors on Jupiter. J. Geophys. Res. B6, 8797-8814.

Ramaprasad, K. R., J. Caldwell, and D. S. McClure (1978). The vibrational overtone spectrum of liquid methane in the visible and near infrared: Applications to planetary studies. Icarus 35, 400-409.

Salahub, D. R., A. E. Foti, and V. H. Smith, Jr. (1978). Molecular orbital study of structural changes on oxidation and reduction of $s_{3}, s_{4}, s_{6}$, and $S_{8}$. J. Amer. Chem. Soc. 100, 7847-7859.

Sato, M. and J. E. Hansen (1979) Jupiter's atmospheric composition and cloud structure deduced from absor.ption bands in reflected sunlight. J. Atmos. Sci. 36, 1133-1167.

Smith, P. H., and M. F. Tomasko (1984). Photometry and polarimetry of Jupiter at large phase angles. II. Polarimetry of the South Tropical Zone, South Equatorial Belt, and the polar regions from the Pioneer 10 and 11 missions. Icarus, in press.

Smith, W. H. (1978). On the ortho-para equilibrium of $\mathrm{H}_{2}$ in the atmospheres of 
the Jovian planets. Icarus $33,210-216$.

Stoker, C. (1983). Vertical structure and convective dynamics of the equatorial region on Jupiter. Ph.D. dissertation, Univ. of Colorado. Stoker. C. (1984). Vertical cloud structure of the equatorial region on Jupiter. Icarus, in press.

Teifel, V. G. (1976). Morphology of molecular absorption on the disk of Jupiter. pp. 441-485 in Jupiter, (T. Gehrels, Ed.), University of Arizona Press, Tucson.

Terrile, R.J. and J.A. Westphal (1977). The vertical cloud structure of Jupiter from 5 um measurements. Icarus 30, 274-281.

Terrile, R.J. and R.F. Beebe (1979). Summary of historical data:

Interpretation of the Pioneer and Voyager cloud configurations in a timedependent framework. Science, 204, 948-951.

Tomasko, M. G., R. A. West, and N. D. Castillo (1978). Photometry and polarimetry of Jupiter at large phase angles I. Analysis of imaging data of a prominent belt and a zone from Pioneer 10. Icarus 33, 558-592.

Trauger, J. T., F. L. Roesler, N. P. Carleton, and W. A. Traub (1973). Observation of HD of Jupiter and the D/H ratio. Astrophys. J. 184, L157L160.

Wallace, L. and G. R. Smith (1977). The interpretation of Jovian methane absorptions. Astrophys. J. 212, 252-261.

West, R. A. (1979a). Spatially resolved methane band photometry of Jupiter I. Absolute reflectivity and center-to-limb variations in the 6190-, 7250-, and $8900-\AA$ bands. Icarus $38,12-33$.

West, R. A. (1979b). Spatially resolved methane band photometry of Jupiter II. Analys is of the South Equatorial Belt and South Tropical Zone reflectivity. Icarus $38,34-53$. 
West, R. A., and M. G. Tomasko (1980). Spatially resolved methane band photometry of Jupiter III. Cloud vertical structures for several axisymmetric bands and the Great Red Spot. Icarus 41, 278-292.

Woodman, J. H., W. D. Cochran, and D. B. Slavsky (1979). Spatially resolved reflectivities of Jupiter during the 1976 opposition. Icarus 37, 73-83. Young, A. T. (1974). Television photometry: The Mariner 9 experience. Icarus $21,262-282$. 
TABLE I

IMAGE DATA

FDS

SCET

FILTER

Day/Hr.Min.sec

$10^{6} \mathrm{~km}$

$\begin{array}{llllllll}16341.50 & 62 / 22.00 .33 & \text { GREEN } & 2.29 & 9.4 & 1.5 & 80.1 & 70.9\end{array}$

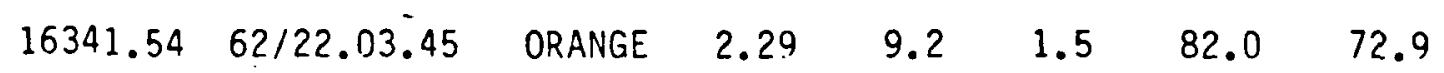

$\begin{array}{lllllllll}16341.58 & 62 / 22.06 .57 & \text { CH4 } & 2.29 & 9.8 & 1.5 & 83.9 & 74.8\end{array}$

$\begin{array}{llllllll}16348.16 & 63 / 03.09 .21 & \text { ORANGE } & 2.02 & 7.4 & 1.3 & 264.6 & 257.6\end{array}$

$\begin{array}{llllllll}16348.20 & 63 / 03.12 .33 & \text { CH4 } & 2.02 & 7.3 & 1.3 & 266.5 & 259.5\end{array}$

$\begin{array}{llllllll}16348.32 & 63 / 03.22 .09 & \text { GREEN } & 2.02 & 7.1 & 1.3 & 272.3 & 265.3\end{array}$

$\begin{array}{llllllll}16373.37 & 63 / 23.25 .09 & \text { GREEN } & 0.89 & 14.1 & 16.0 & 265.0 & 273.2\end{array}$

$\begin{array}{llllllll}16373.41 & 63 / 23.29 .21 & \text { ORANGE } & 0.89 & 14.2 & 11.5 & 266.7 & 275.1\end{array}$

$\begin{array}{llllllll}16373.45 & 63 / 23.32 .33 & \mathrm{CH} 4 & 0.88 & 13.9 & 15.2 & 263.1 & 277.0\end{array}$

$\begin{array}{llllllll}16378.04 & 64 / 02.59 .45 & \text { GREEN } & 0.69 & 25.8 & 10.8 & 37.5 & 42.3\end{array}$

$\begin{array}{llllllll}16378.08 & 64 / 03.02 .57 & \text { ORANGE } & 0.68 & 25.8 & 12.3 & 36.4 & 44.2\end{array}$

$\begin{array}{llllllll}16378.12 & 64 / 03.06 .09 & \text { CH4 } & 0.68 & 25.7 & 8.4 & 35.9 & 46.1\end{array}$ 


$\begin{array}{rrrrrrrr}16379.44 & 64 / 04.19 .45 & \text { GREEN } & 0.61 & 30.9 & -19.9 & 77.5 & 90.6 \\ 16379.48 & 64 / 04.22 .57 & \text { ORANGE } & 0.60 & 31.3 & -19.5 & 80.5 & 92.6 \\ 16379.52 & 64 / 04.26 .09 & \text { CH4 } & 0.60 & 31.9 & -19.4 & 84.7 & 94.5\end{array}$

FDS is the Flight Data Subsystem count for each image. SCET is the FMT Spacecraft Event Time of frame readout. The Lat. and Lon. values are planetographic latitude and System III longitude of the subspacecraft point for the first six images, and the latitude, longitude coordinates of the center of the image for the remaining images. $L_{S}$ is the subsolar System III longitude. 
TABLE II

SPOT MEASUREMENTS ON HIGH RESOLUTION FRAMES

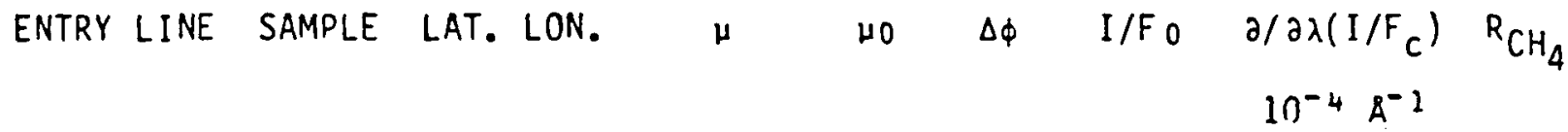

BARGE IMAGE (FDS 16373.41)

$\begin{array}{lllllllllll}1 & 394 & 470 & 14.6 & 269.0 & 0.9509 & 0.9610 & 125.3 & 0.448 & 1.89 & 0.910\end{array}$

$\begin{array}{lllllllllll}2 & 264 & 252 & 18.6 & 279.8 & 0.8826 & 0.9493 & 145.2 & 0.766 & -1.61 & 0.906\end{array}$

$\begin{array}{lllllllllll}3 & 296 & 468 & 19.3 & 270.1 & 0.9197 & 0.9402 & 137.1 & 0.776 & -1.64 & 0.909\end{array}$

$\begin{array}{lllllllllll}4 & 334 & 658 & 19.3 & 261.6 & 0.9305 & 0.9135 & -140.9 & 0.765 & -1.64 & 0.904\end{array}$

$\begin{array}{lllllllllll}5 & 368 & 842 & 19.5 & 253.3 & 0.9169 & 0.8675 & -150.6 & 0.746 & -1.62 & 0.900\end{array}$

$\begin{array}{lllllllllll}6 & 414 & 512 & 15.0 & 262.8 & 0.9569 & 0.9395 & 131.8 & 0.672 & -0.19 & 0.900\end{array}$

$\begin{array}{lllllllllll}7 & 490 & 446 & 9.8 & 269.0 & 0.9720 & 0.9778 & 105.3 & 0.603 & -0.57 & 0.925\end{array}$

$\begin{array}{lllllllllll}8 & 444 & 230 & 9.9 & 278.6 & 0.9325 & 0.9861 & 132.3 & 0.615 & -0.34 & 0.911\end{array}$

$\begin{array}{lllllllllll}9 & 560 & 844 & 10.3 & 252.0 & 0.9612 & 0.8942 & -152.8 & 0.567 & -0.58 & 0.889\end{array}$

$\begin{array}{lllllllllll}10 & 430 & 580 & 13.9 & 264.0 & 0.9619 & 0.9487 & 127.4 & 0.666 & 0.17 & 0.874\end{array}$

$\begin{array}{lllllllllll}11 & 650 & 852 & 6.1 & 251.0 & 0.9703 & 0.8956 & -159.7 & 0.522 & -1.48 & 0.901\end{array}$

$\begin{array}{lllllllllll}12 & 442 & 572 & 13.2 & 264.2 & 0.9650 & 0.9520 & 125.2 & 0.646 & -0.16 & 0.869\end{array}$ 
PLUME IMAGE (FDS 16378.08)

$\begin{array}{lllllllllll}13 & 664 & 510 & 5.2 & 35.4 & 0.9519 & 0.9833 & 51.4 & 0.805 & -0.43 & 0.840\end{array}$

$\begin{array}{llllllllll}14 & 718 & 310 & 3.3 & 34.5: 0.9606 & 0.9828 & 35.0 & 0.795 & -0.74 & 0.896\end{array}$

$\begin{array}{lllllllllll}15 & 724 & 420 & 2.8 & 38.2 & 0.9406 & 0.9926 & 33.5 & 0.805 & -0.62 & 0.875\end{array}$

$\begin{array}{lllllllllll}16 & 718 & 532 & 3.3 & 34.5 & 0.9606 & 0.9828 & 35.0 & 0.757 & -0.73 & 0.882\end{array}$

$\begin{array}{lllllllllll}17 & 694 & 620 & 4.4 & 31.7 & 0.9717 & 0.9719^{\circ} & 45.3 & 0.587 & -1.58 & 0.871\end{array}$

$\begin{array}{lllllllllll}18 & 698 & 670 & 4.4 & 30.1 & 0.9771 & 0.9651 & 47.5 & 0.556 & -1.78 & 0.868\end{array}$

$\begin{array}{lllllllllll}19 & 870 & 382 & -2.6 & 39.1 & 0.9387 & 0.9936 & 30.3 & 0.640 & -0.22 & 0.847\end{array}$

$\begin{array}{lllllllllll}20 & 880 & 462 & -2.8 & 36.4 & 0.9552 & 0.9876 & 24.0 & 0.670 & -0.16 & 0.860\end{array}$

$\begin{array}{lllllllllll}21 & 886 & 570 & -2.7 & 32.8 & 0.9732 & 0.9767 & 18.5 & 0.657 & -0.56 & 0.868\end{array}$

$\begin{array}{lllllllllll}22 & 920 & 770 & -3.4 & 26.3 & 0.9939 & 0.9463 & 26.8 & 0.622 & -0.13 & 0.887\end{array}$

$\begin{array}{lllllllllll}23 & 506 & 512 & 11.0 & 36.0 & 0.9288 & 0.9716 & 88.7 & 0.654 & -1.13 & 0.920\end{array}$

$\begin{array}{lllllllllll}24 & 422 & 306 & 13.7 & 43.5 & 0.8658 & 0.9734 & 117.0 & 0.717 & -0.44 & 0.840\end{array}$

$\begin{array}{lllllllllll}25 & 408 & 460 & 14.6 & 38.2 & 0.8981 & 0.9638 & 106.4 & 0.790 & -0.71 & 0.871\end{array}$

$\begin{array}{lllllllllll}26 & 470 & 612 & 12.6 & 32.8 & 0.9367 & 0.9566 & 93.8 & 0.680 & -0.96 & 0.869\end{array}$

$\begin{array}{lllllllllll}27 & 298 & 206 & 18.3 & 48.2 & 0.8005 & 0.9507 & 134.6 & 0.750 & -1.32 & 0.899\end{array}$

$\begin{array}{lllllllllll}28 & 300 & 322 & 18.4 & 43.9 & 0.8360 & 0.9516 & 125.9 & 0.772 & -1.36 & 0.908\end{array}$

$\begin{array}{lllllllllll}29 & 308 & 452 & 18.4 & 39.1 & 0.8692 & 0.9473 & 118.0 & 0.743 & -1.55 & 0.908\end{array}$

$\begin{array}{lllllllllll}30 & 312 & 566 & 18.5 & 35.2 & 0.8902 & 0.9376 & 114.7 & 0.743 & -1.39 & 0.904\end{array}$

$\begin{array}{lllllllllll}31 & 322 & 682 & 18.4 & 31.2 & 0.9084 & 0.9251 & 113.5 & 0.767 & -1.29 & 0.905\end{array}$ $\begin{array}{lllllllllll}32 & 350 & 826 & 17.7 & 26.2 & 0.9267 & 0.9059 & 115.2 & 0.727 & -1.63 & 0.904\end{array}$

GREAT RED SPOT IMAGE (FDS 16379.48)

$\begin{array}{lllllllllll}33 & 496 & 492 & -19.4 & 80.8 & 0.8953 & 0.9172 & 99.6 & 0.725 & -1.19 & 0.940\end{array}$

$\begin{array}{lllllllllll}34 & 542 & 552 & -20.7 & 78.8 & 0.8984 & 0.9022 & 102.9 & 0.597 & 0.28 & 0.868\end{array}$ 


$\begin{array}{lllllllllll}35 & 552 & 764 & -20.1 & 72.4 & 0.9289 & 0.8733 & 105.4 & 0.720 & -0.37 & 0.928 \\ 36 & 616 & 808 & -22.0 & 70.9 & 0.9203 & 0.8526 & 111.8 & 0.697 & -0.05 & 0.934 \\ 37 & 580 & 842 & -20.7 & 69.9 & 0.9316 & 0.8546 & 110.2 & 0.696 & -0.21 & 0.923 \\ 38 & 444 & 628 & -17.1 & 76.6 & 0.9297 & 0.9121 & 92.5 & 0.557 & -0.44 & 0.846 \\ 39 & 540 & 328 & -21.6 & 86.1 & 0.8463 & 0.9191 & 108.9 & 0.521 & -0.52 & 0.847 \\ 40 & 540 & 260 & -21.9 & 88.4 & 0.8263 & 0.9213 & 112.2 & 0.601 & -0.50 & 0.879 \\ 41 & 308 & 414 & -13.5 & 83.4 & 0.9062 & 0.9549 & 81.7 & 0.730 & -1.32 & 0.918 \\ 42 & 190 & 302 & -10.2 & 87.1 & 0.8884 & 0.9765 & 75.8 & 0.679 & -1.08 & 0.929 \\ 43 & 144 & 368 & -8.4 & 84.5 & 0.9127 & 0.9760 & 59.5 & 0.567 & -0.02 & 0.888 \\ 44 & 174 & 544 & -8.8 & 79.7 & 0.9446 & 0.9586 & 54.0 & 0.556 & 0.01 & 0.877 \\ 45 & 194 & 700 & -8.8 & 75.1 & 0.9680 & 0.9365 & 54.4 & 0.520 & 0.46 & 0.869 \\ 46 & 798 & 365 & -30.3 & 85.4 & 0.7856 & 0.9505 & 123.6 & 0.635 & -0.65 & 0.928 \\ 47 & 846 & 494 & -31.4 & 80.9 & 0.8021 & 0.8288 & 124.1 & 0.571 & -0.63 & 0.928 \\ 48 & 836 & 612 & -30.5 & 76.9 & 0.8302 & 0.8216 & 123.1 & 0.580 & -0.35 & 0.938\end{array}$


TABLE III

$\mathrm{H}_{2}$ Quadrupole Line Parameters at STP

Line Wavelength So Pressure Broadening Coefficient

(A) $\left(10^{-4} \mathrm{~cm}^{-1}\right) \quad\left(\mathrm{cm}^{-1} / \mathrm{atm}\right)$

$\begin{array}{rrrr}3-0 S(0) & 8274.9 & 2.8 & 0.0062 \\ 3-0 S(1) & 8152.9 & 12.1 & 0.0070\end{array}$

$\begin{array}{llll}4-0 S(0) & 6436.8 & 0.22 & 0.0080 \\ 4-0 S(1) & 6367.3 & 1.42 & 0.0085\end{array}$


FIGURE CAPTIONS

Figure 1. . TOP: Spectrum of Jupiter's North Tropical Zone-taken in 1979 by Cochran et al. (1981). CENTER: Responsivity functions for the green, orange and methane filters normalized to unity at their maximum values. BOTTOM: Methane absorption coefficients reported by Giver (1978).

Figure 2. Cylindrical projection map of orange frames 16341.54 and 16348.20. Planetographic latitude and System III longitude are marked. The grey scale indicates I/F.

Figure 3. Cylindrical projection map of the continuum spectral gradient, $\partial / \partial \lambda\left(I / F_{C}\right)$, at the effective wavelength of the orange filter $(6046 \AA)$. The grey scale calibration is in units of $10^{-6} \AA^{-1}$.

Figure 4. Cylindrical projection map of the methane/continuum ratio, ${ }^{\mathrm{R}} \mathrm{CH}_{4}$. Figure 5. Orange reflectivity and $6190 \AA$ equivalent width $W \&$ along a $10^{\circ}$ wide longitude track centered at $255^{\circ}$ System III longitude.

Figure 6. Equivalent width vs. I/F from Fig. 5.

Figure 7. Equivalent width of the 6190 \& band as a function of longitude. The data in Fig. 4 were convolved with a window $16^{\circ}$ in longitude by $11^{\circ}$ in latitude "centered at the equator.

Figure 8. Orange image 16373.41. The grey scale indicates I/F. Tick marks along the border are at 100 pixel intervals.

Figure 9. Continuum spectral gradient for the region in Fig. 8. The grey scale calibration is in units of $10^{-6} \mathrm{~A}^{-1}$.

Figure 10. Methane/continuum ratio for the region in Fig. 8 . 
Figure 11. Contours of 6190 \& methane band equivalent for the region in Fig. 10. The units are $\AA$, and the contour interval is 2 . Local maxima and minima are indicated. The contours were generated from contiguous $20 \times 20$ pixel averages.

Figure 12. Orange image 16378.08

Figure 13. Spectral gradient for the region in Figure 12.

Figure 14. Methane/continuum ratio for the region in Fig. 12.

Figure 15. Contours of 6190 A methane band equivalent width in \& for the region in Fig. 12.

Figure 16. Orange image 16379.48

Figure 17. Spectral gradient for the region in Figure 16.

Figure 18. Methane/continuum ratio for the region in Fig. 16.

Figure 19. Contours of 6190 \& methane band equivalent width in $\&$ for the region in Fig. 16.

Figure 20. Lahoratory spectral reflectance measurements of elemental sulfur and phosphorus deposits formed at several temperatures (from $\mathrm{J}$. Gradie, private communication, 1982.)

Figure 21. Contours of methane $6190 \AA$ band equivalent width $(\AA)$ in the central portion of image 16737.41 . The contour interval is $2 \AA$. Local maxima and minima are indicated. The map was constructed from contiguous $6 \times 6$ pixel averages bounded by lines 400-700 and samples 400-700. The major tick marks are spaced at 30 pixel intervals.

Figure 22. Contours of 5 um brightness temperature (averaged over the spectral interval $2000-2050 \mathrm{~cm}^{-1}$ ) from the IRIS raster scan map. The $0.25^{\circ}$ diameter IRIS field of view size is indicated. 
Figure 23. Molecular hydrogen para fraction determined from IRIS measurements.

Figure 24. Contours of methane $6190 \&$ band equivalent width $(A)$ in the central portion of image 16738.08. The contour interval is $2 \AA$. Local maxima and minima are indicated. The map was constructed from contiguous $6 \times 6$ pixel averages bounded by lines $400-700$ and samples 400-700. The major tick marks are spaced at 30 pixel intervals.

Figure 25. Contours of $5 \mathrm{~mm}$ brightness temperature from a second IRIS raster scan map.

Figure 26. Molecular hydrogen para fraction determined from IRIS measurements.

Figure 27. Schematic model atmosphere structure showing the pressuretemperature profile for Jupiter's NEB derived by Kunde et al. (1982), and the locations of the cloud and haze layers studied in the present work. The parameter $P$, the pressure at the top of lower cloud (identified as either $\mathrm{NH}_{4} \mathrm{SH}$ or $\mathrm{H}_{2} \mathrm{O}$ ) is one of the free parameters of the model (see text).

Figure 28. Methane/continuum ratio $\mathrm{R}_{\mathrm{CH}_{4}}$ as a function of optical depth of the ammonia cloud and $P$, the pressure of the lower cloud top. a... Values of other model parameters are $\omega_{c l o u d}=0.998$, and thaze (at $250 \mathrm{mbar})=1.0$. These results were obtained for the scattering geometry values of entry 13 in Table II and are relevant to the bright plume head and NTrZ in Figs. 12-15.

Figure 29. Same as Fig. 28 except thaze $=0.1$.

Figure 30. Same as Fig. 29 except $\omega_{c l \text { oud }}=0.991$ (appropriate for the hot spot regions in Figs. 22 and 25 ). 
Figure 31. Same as Fig. 28 except $\omega_{c l o u d}=0.97$, and the scattering geometry values of entry 1 in Table II were used instead of values for entry 13. These results apply to the dark barge feature in Figs. $8-11$

Figure 32. Equivalent width $(\mathrm{m} \AA)$ of the 4-0 $S(1)$ hydrogen quadrupole line for equilibrium $\mathrm{H}_{2}$. Scattering geometry and model parameters are identical to those for Fig. $29\left(\omega_{\text {cloud }}=0.998 ; \tau_{\text {haze }}=0.1\right)$.

Figure 33. Equivalent width $(\mathrm{m} \AA)$ for the 3-0 $S(1)$ hydrogen quadrupole line for equilibrium $\mathrm{H}_{2}$. Same model parameters as in Fig. 32.

Figure 34. Ratio of 3-0 $S(1) / S(0)$ hydrogen quadrupole equivalent width for equilibrium $\mathrm{H}_{2}$. Model parameters are identical to those in Figs. 33 and 34.

Figure 35. Same as Fig. 34 except for normal $\mathrm{H}_{2}$.

Figure 36. Synthetic spectra for (solid curve) full resolution calculation convolved with the IRIS spectral point spread function, gas absorption only; (dashed curve) spectrum calculated from flux weighted averaged absorption coefficients at $2 \mathrm{~cm}^{-1}$ intervals, gas absorption only; (dotted curve) same as dashed curve but including $\tau_{\mathrm{NH}_{3}}+$ haze $=1.5, \omega=0.865$, and $P=6.08$ bar. Spectra are calculated for nadir viewing.

Figure 37. Brightness temperature at $5 \mathrm{um}$ as a function of ${ }^{\tau} \mathrm{NH}_{3}$ and $\mathrm{P}$ for a cloud composed of large particles whose extinction coefficients and phase functions are independent of wavelength between 0.619 and $5 \mathrm{um}$. 
Figure 38. Brightness temperature at $5 \mathrm{um}$ for an ammonia cloud composed of 1 um particles. To facilitate comparison with Figs. 28-31, the parameter $\tau_{\mathrm{NH}_{3}}$ is the optical depth of the ammonia cloud at 6190 A. To find the corresponding optirat: depth at $5 \mathrm{~mm}$ for this case multiply the abscissa value by 0.2 . 

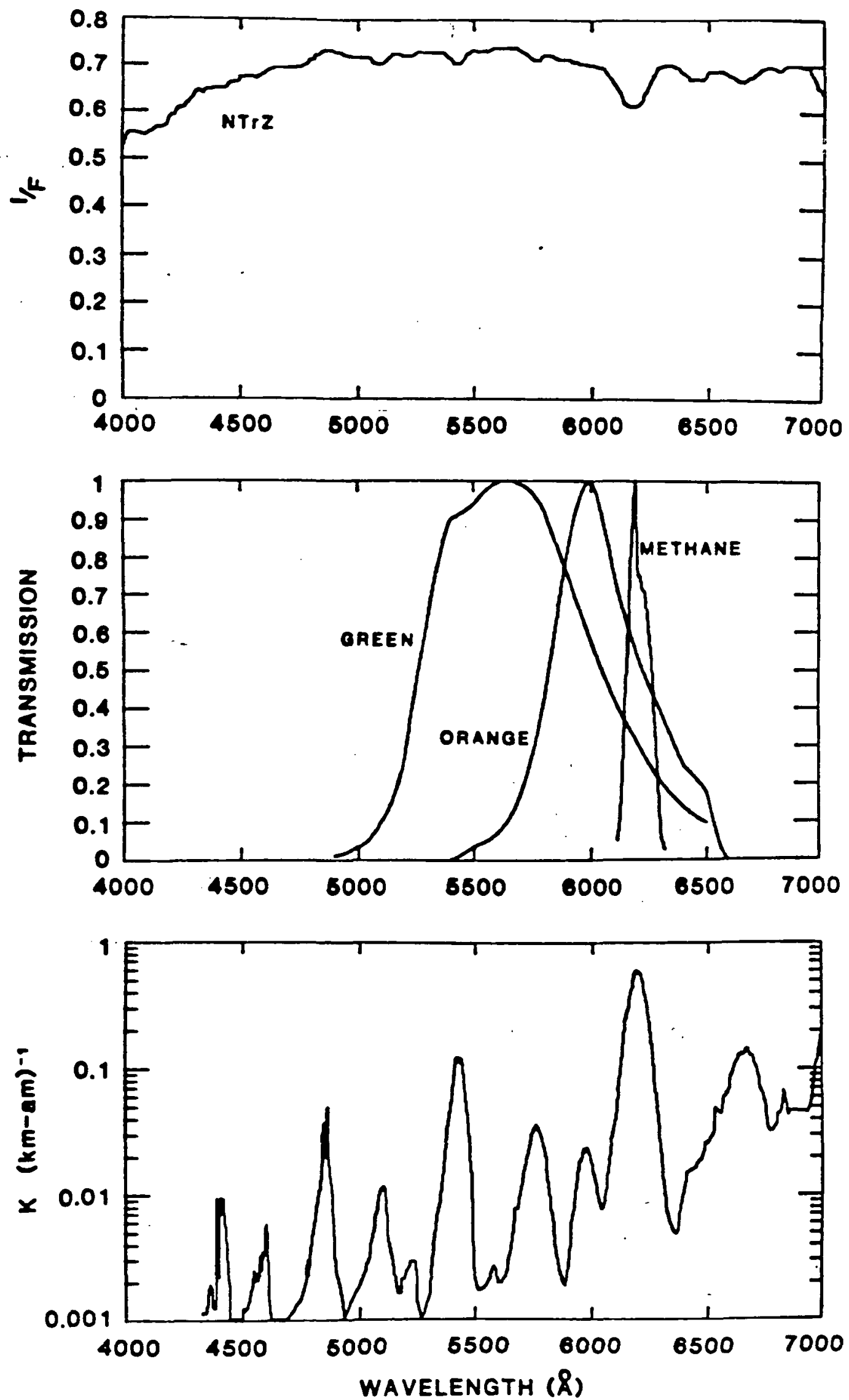

Fig. 1 


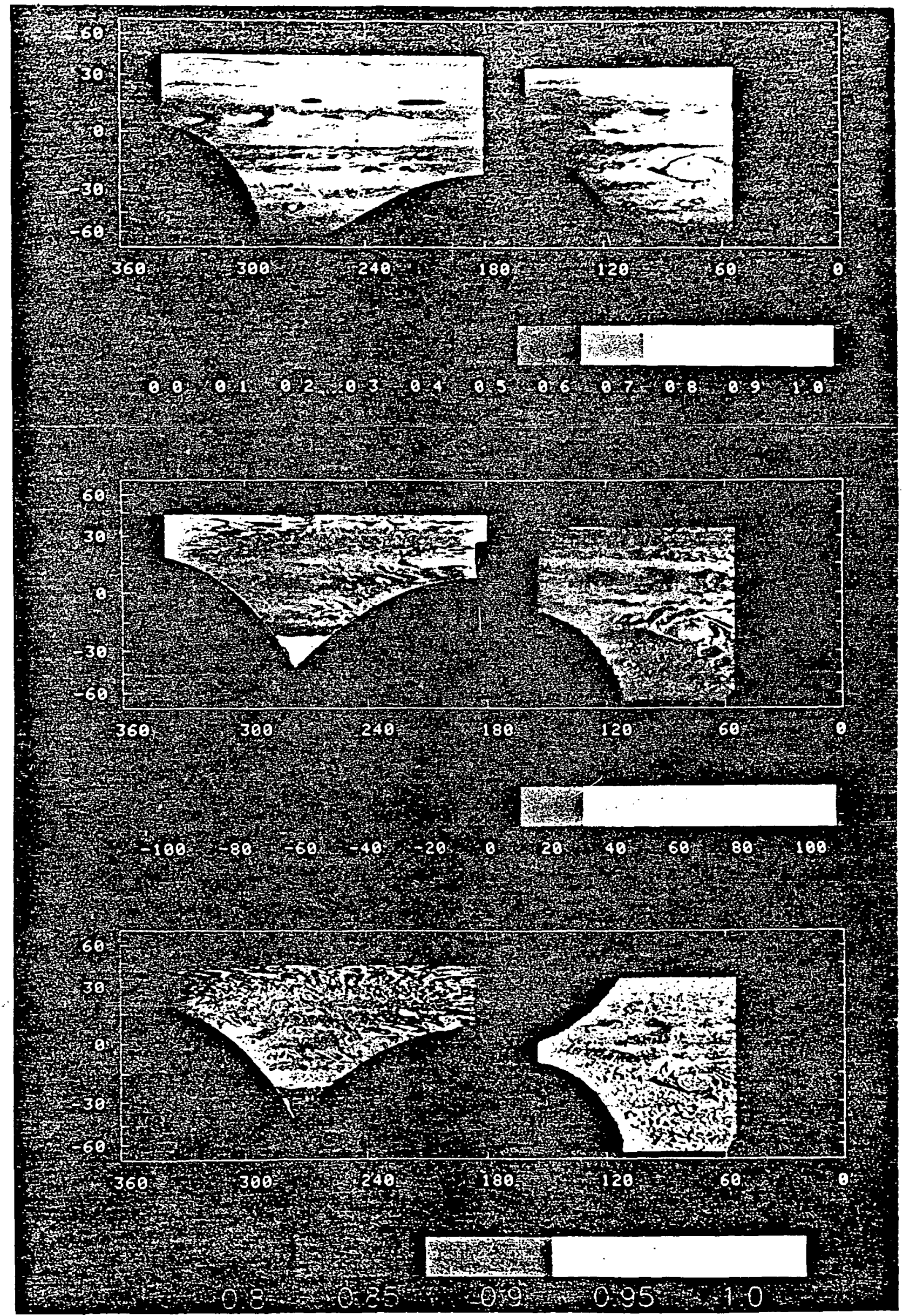




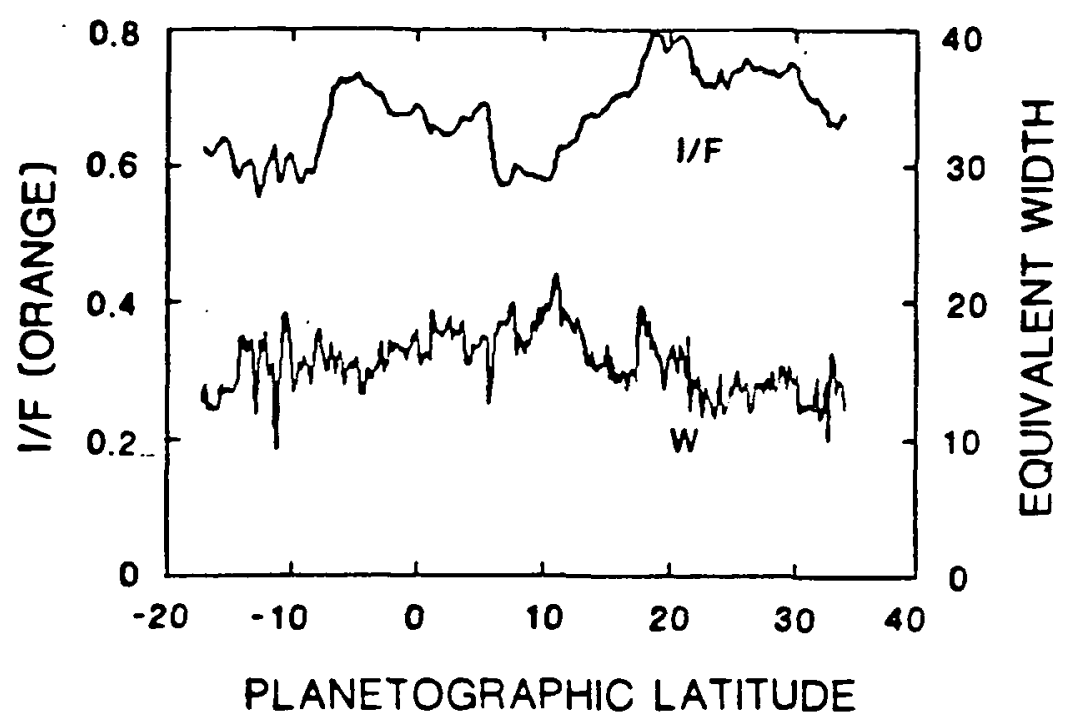

Figure 5

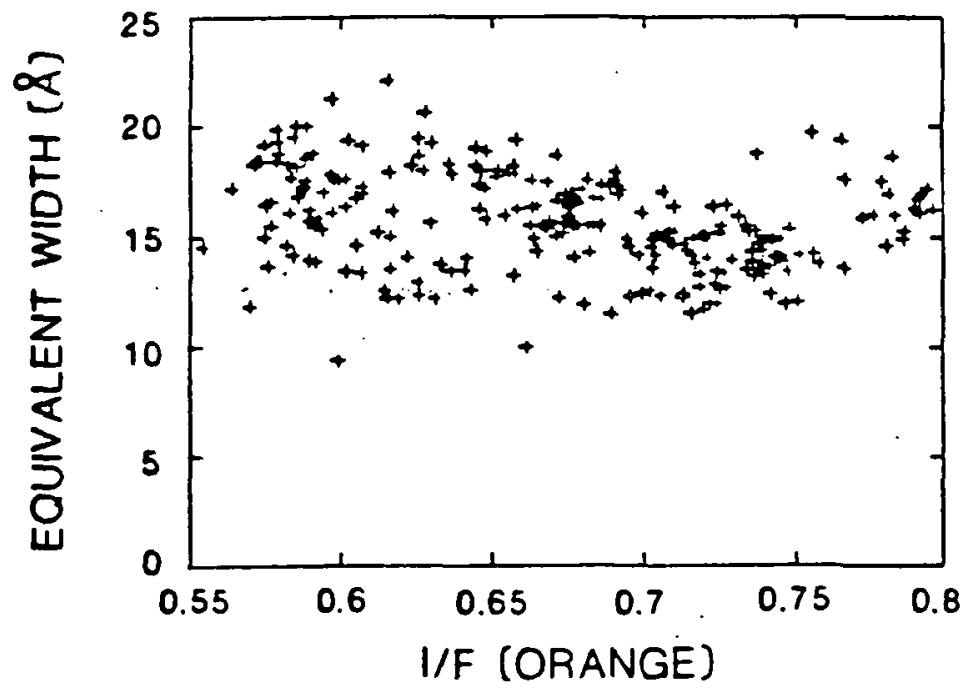

Figure 6 
SYSTEM I LONGITUDE

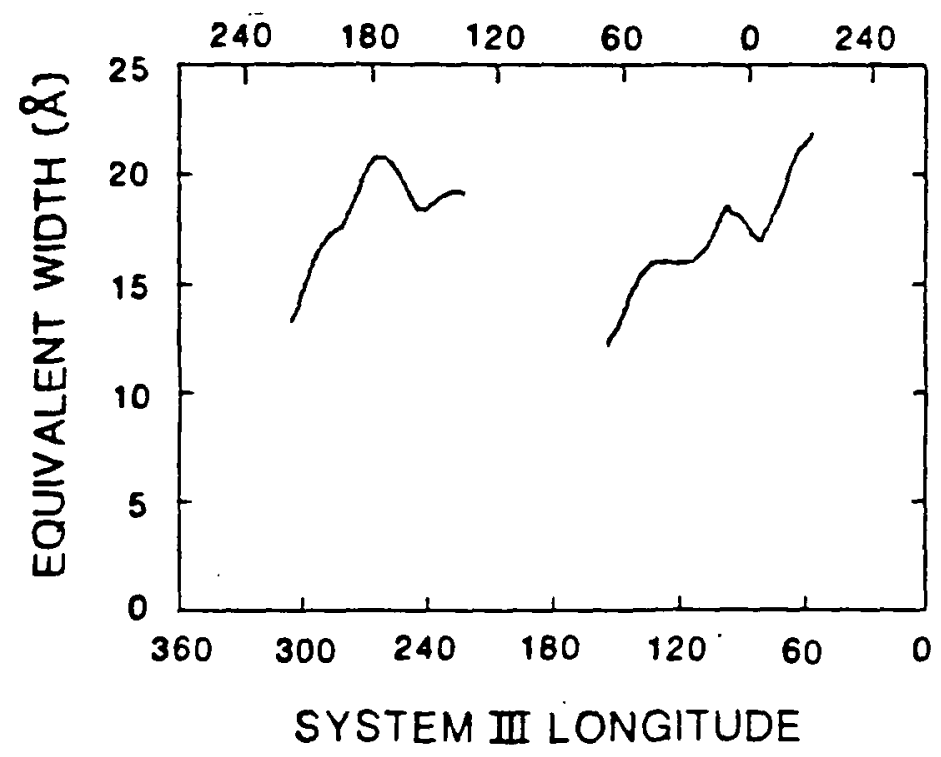

Figure 7 


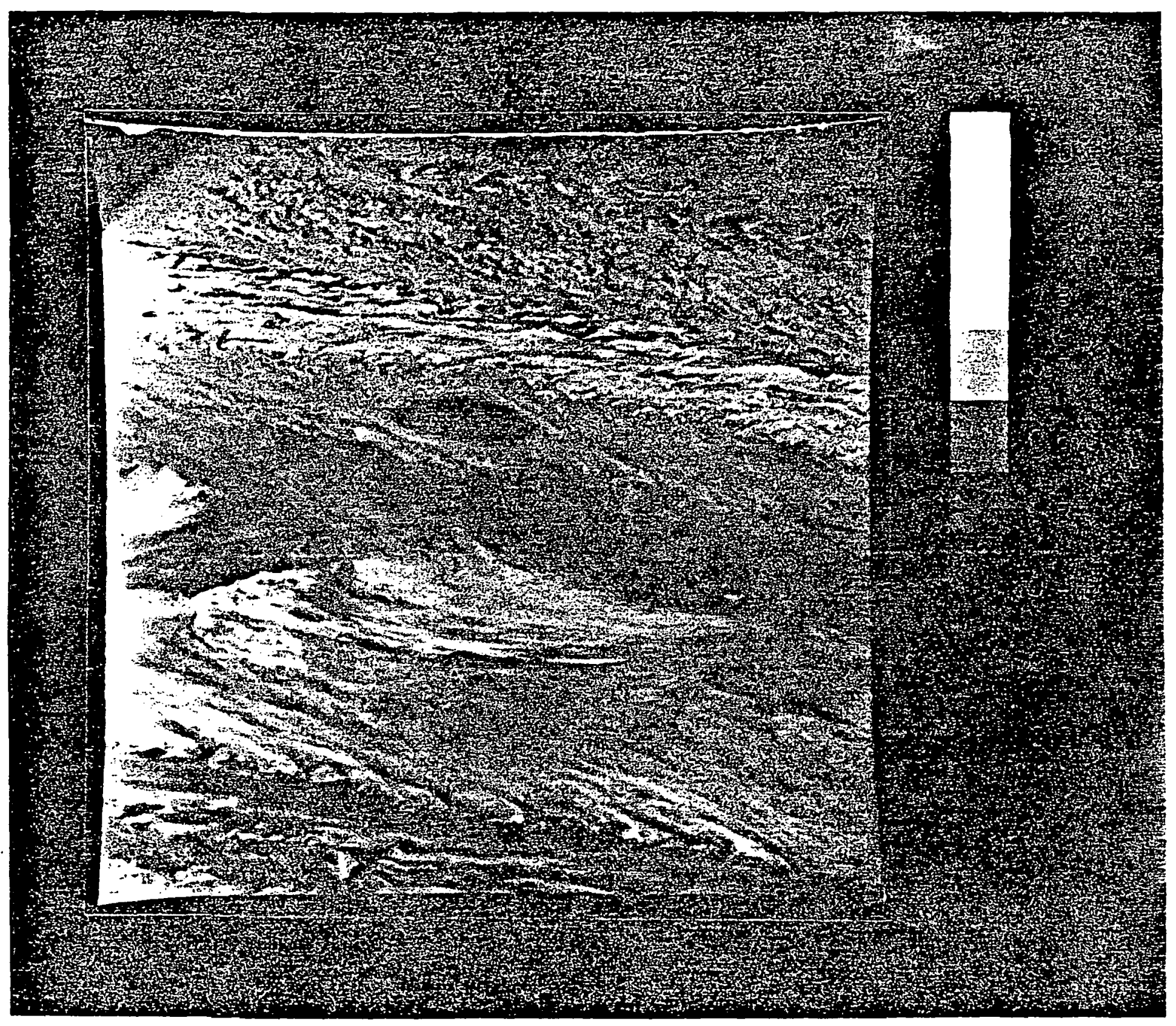




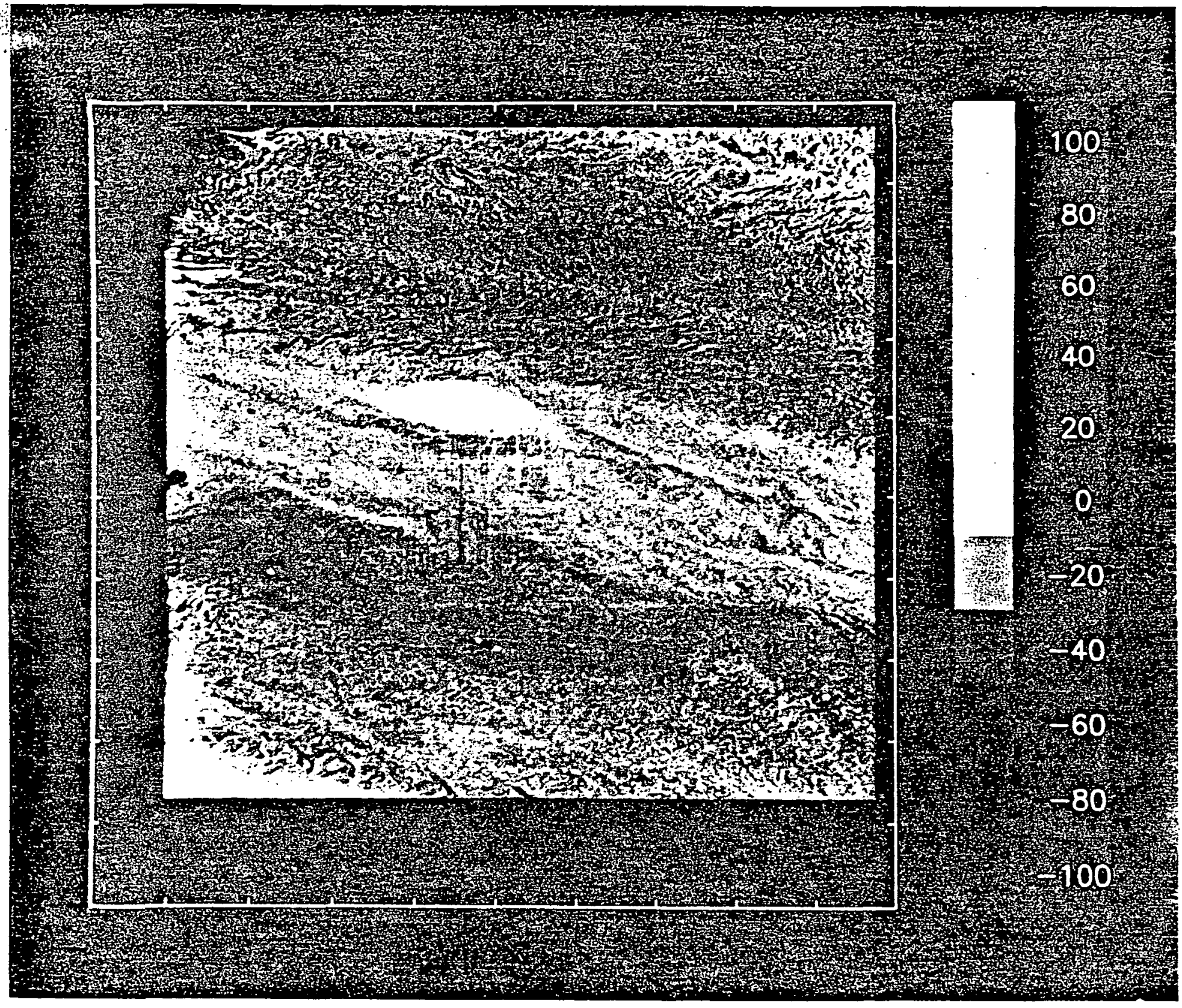




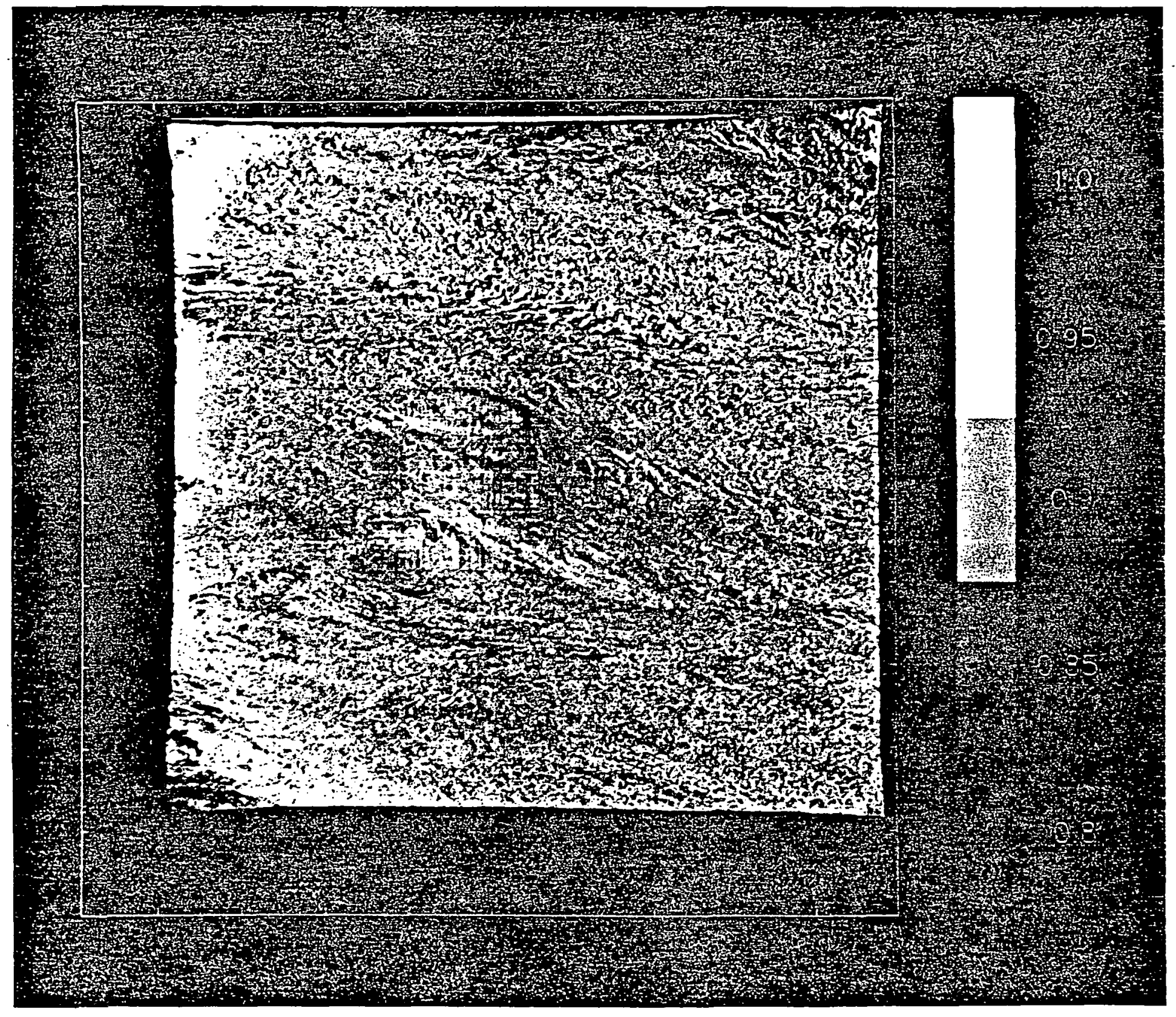




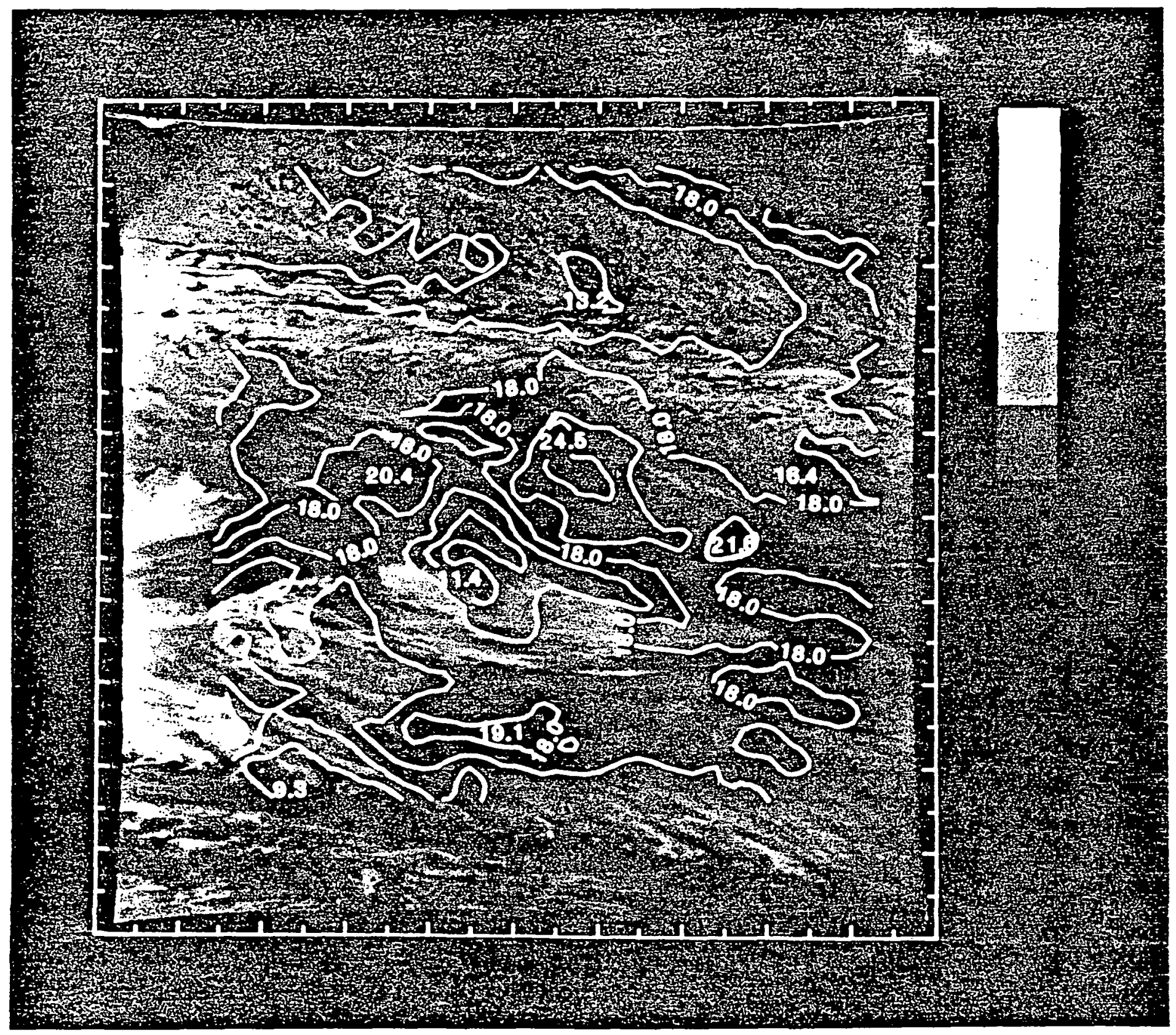




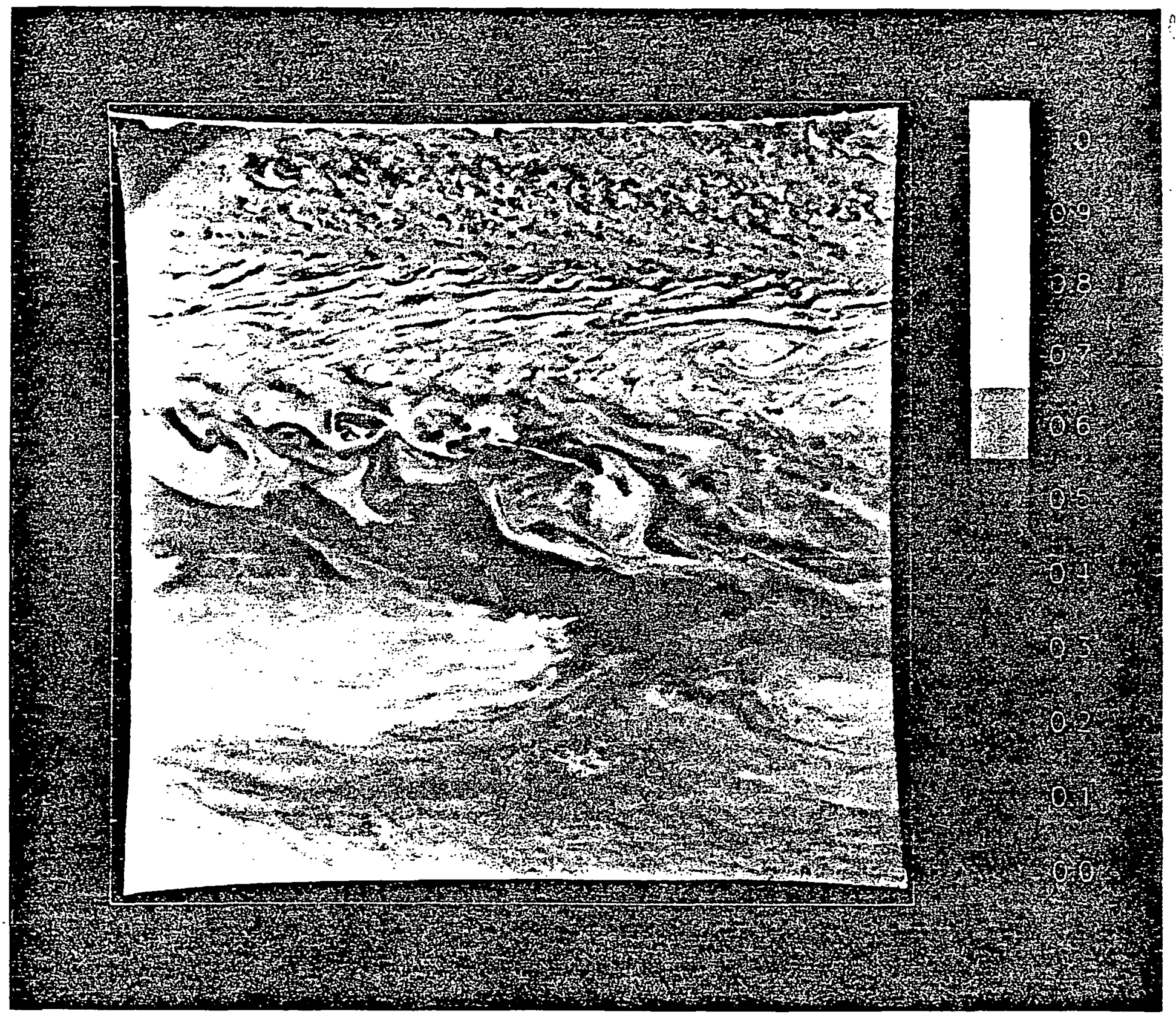




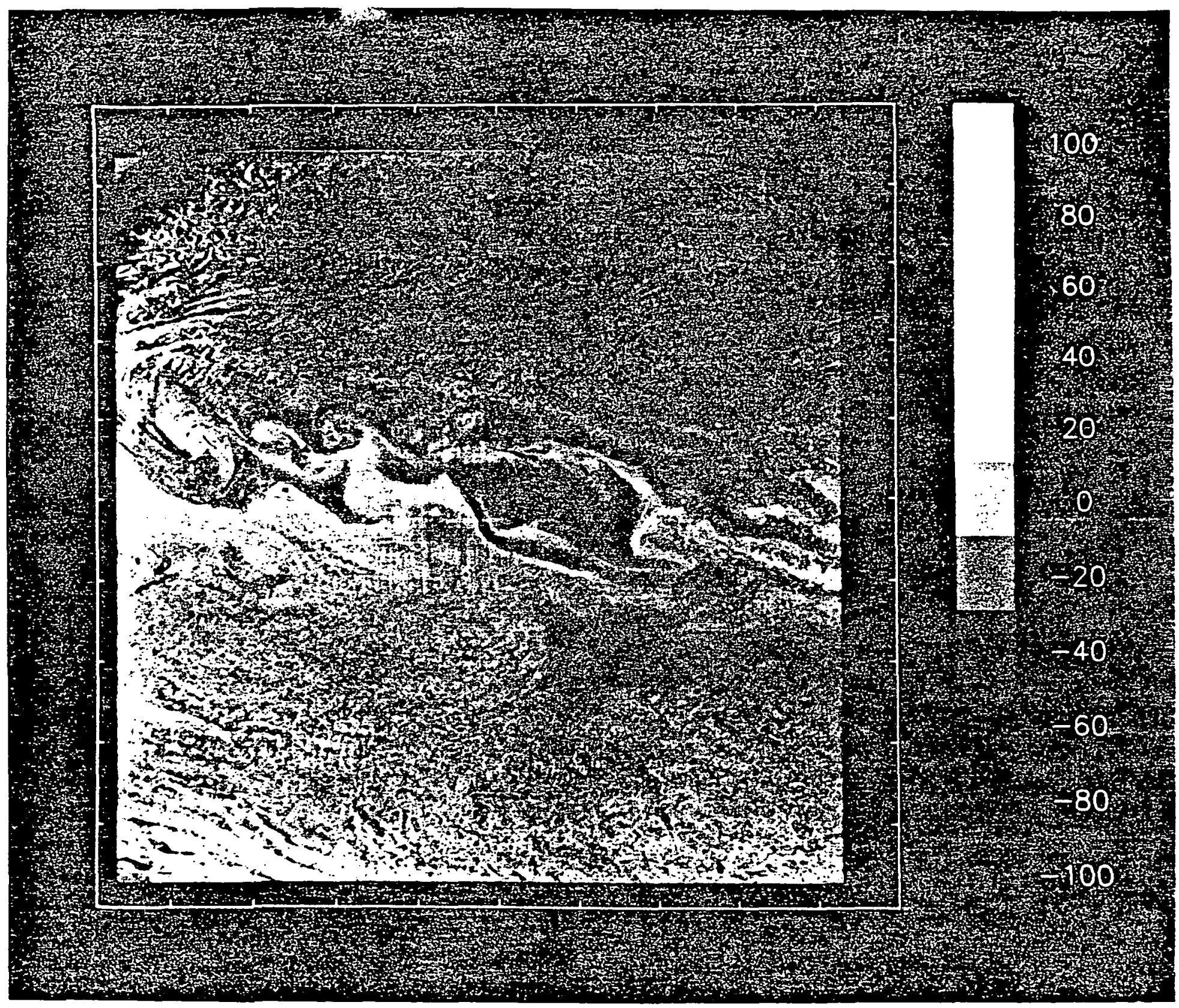




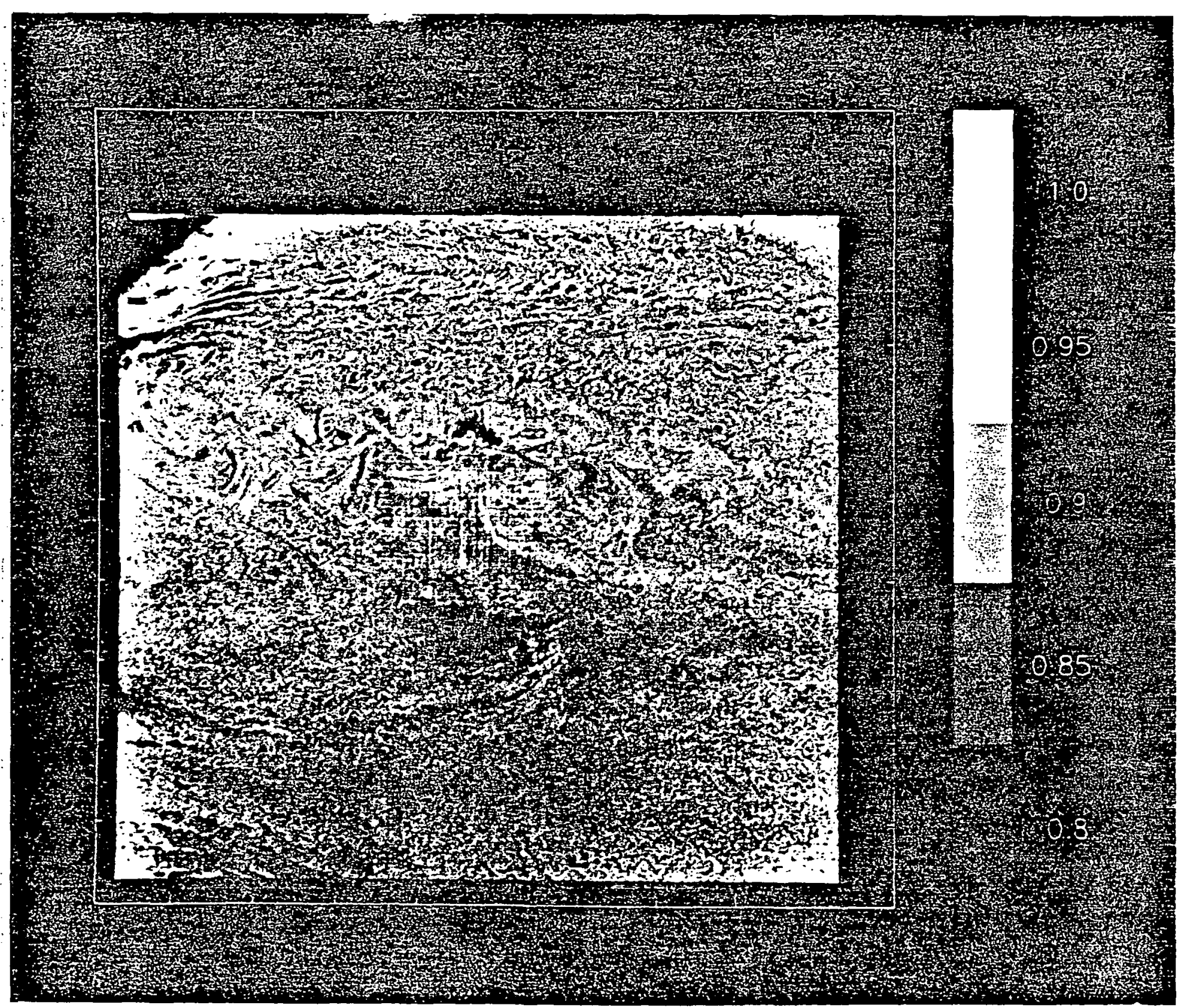




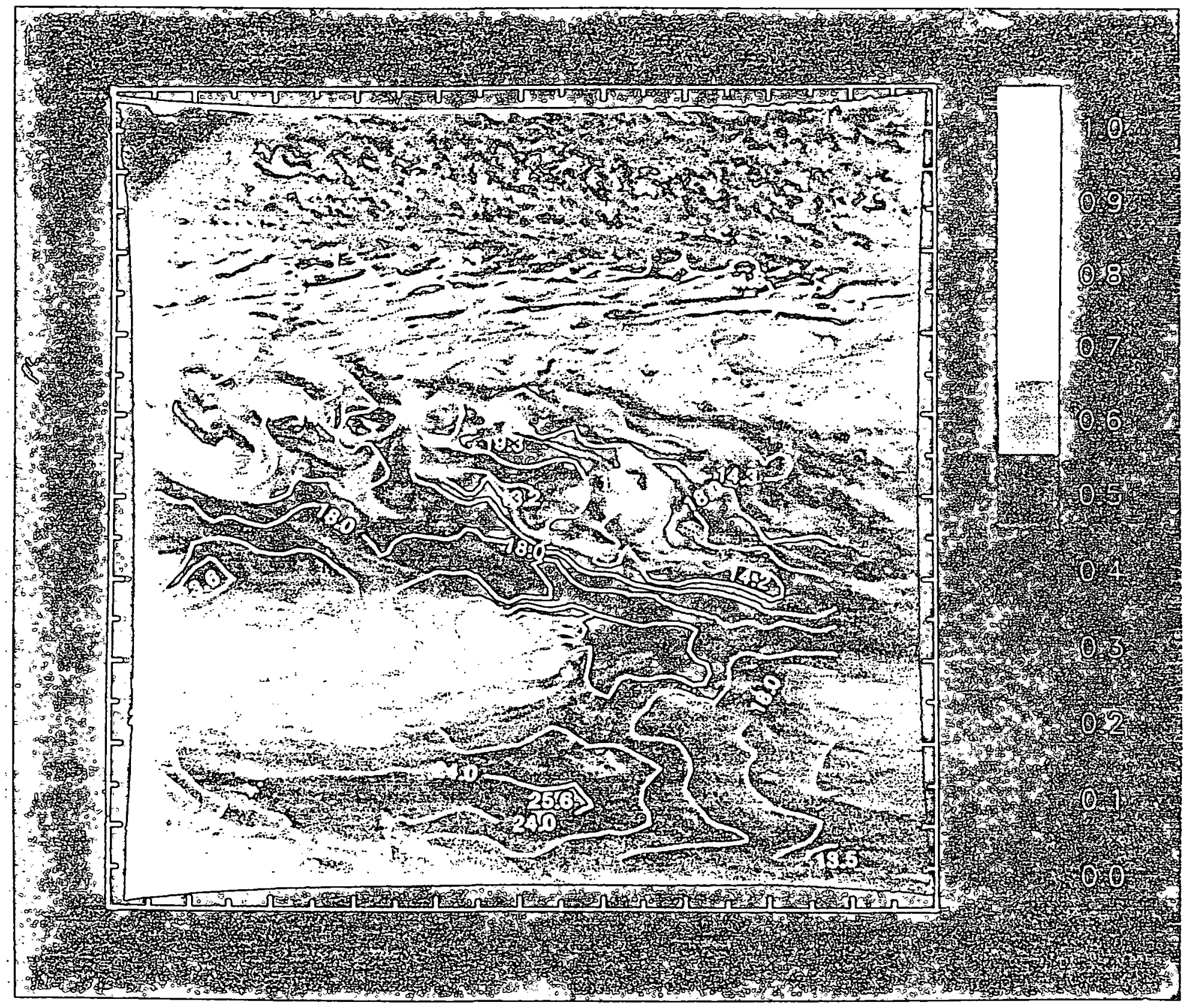




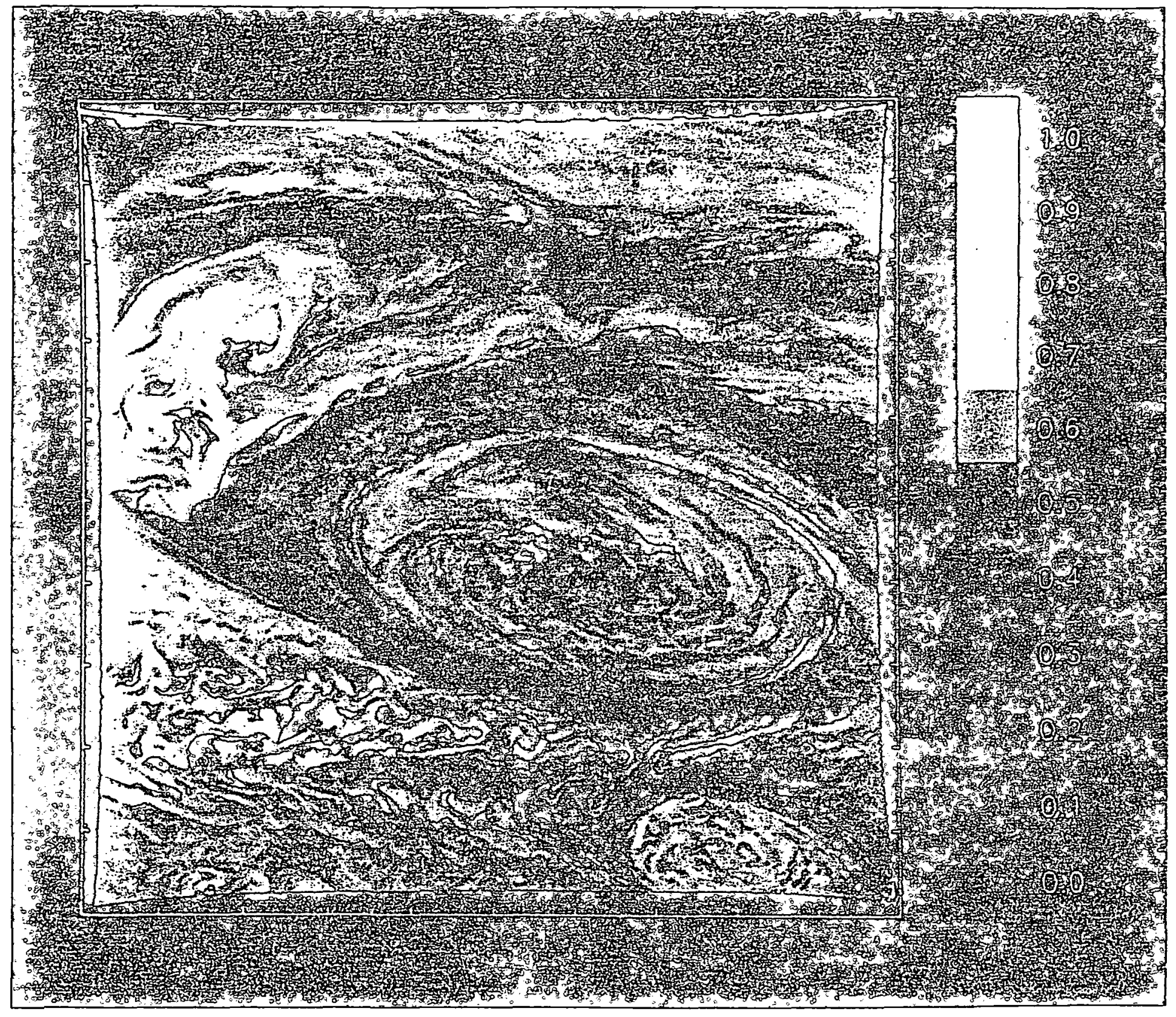




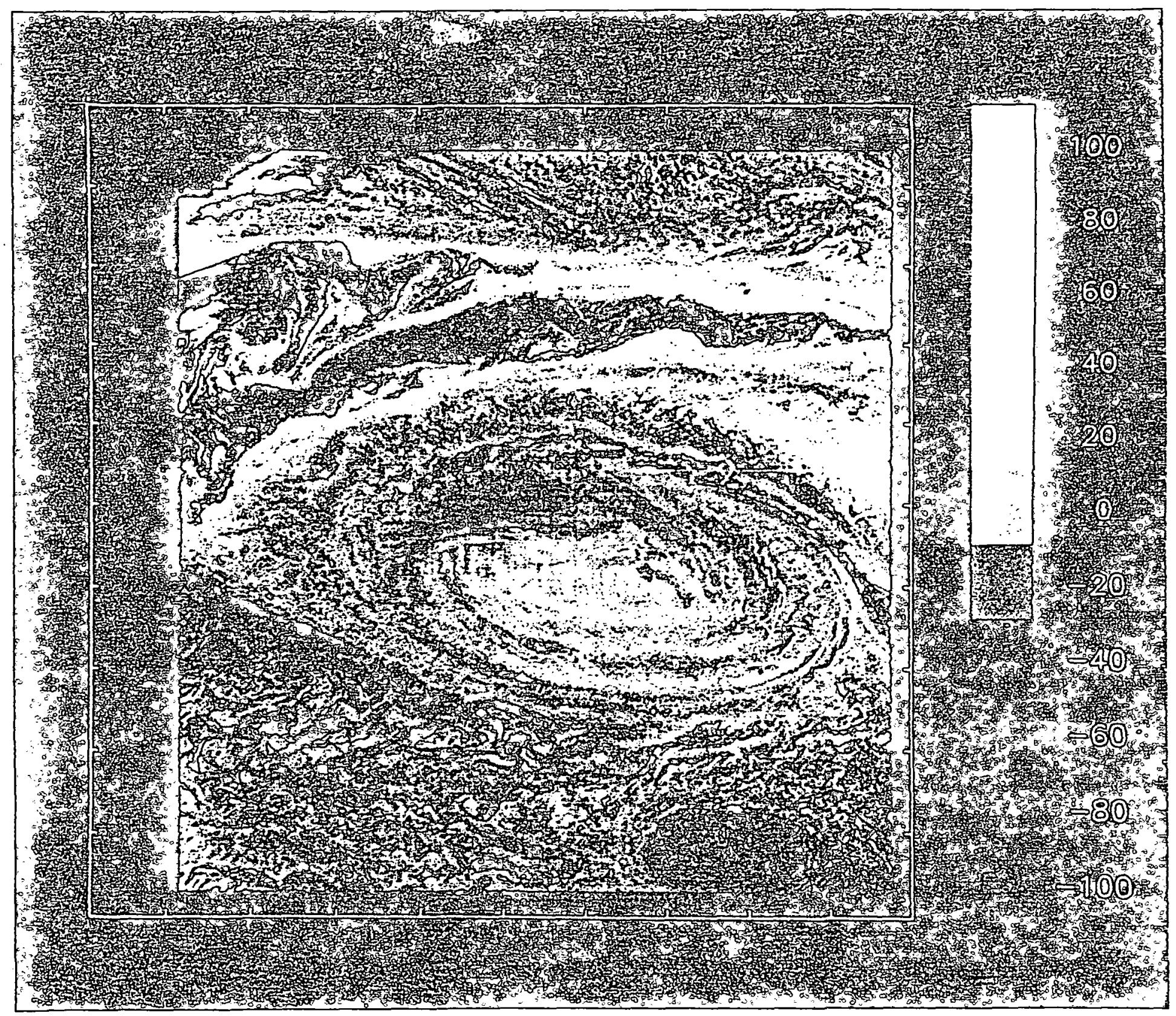




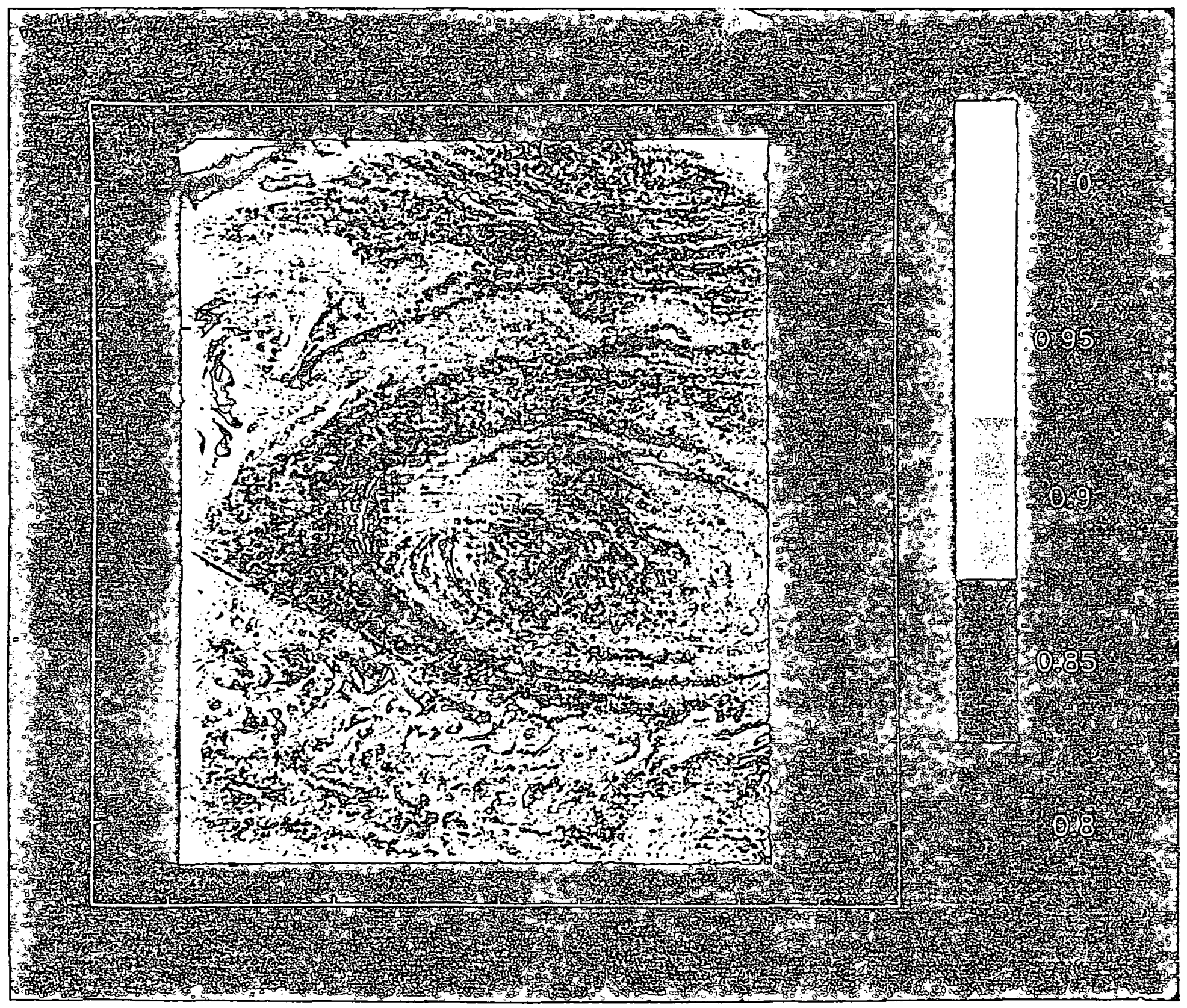




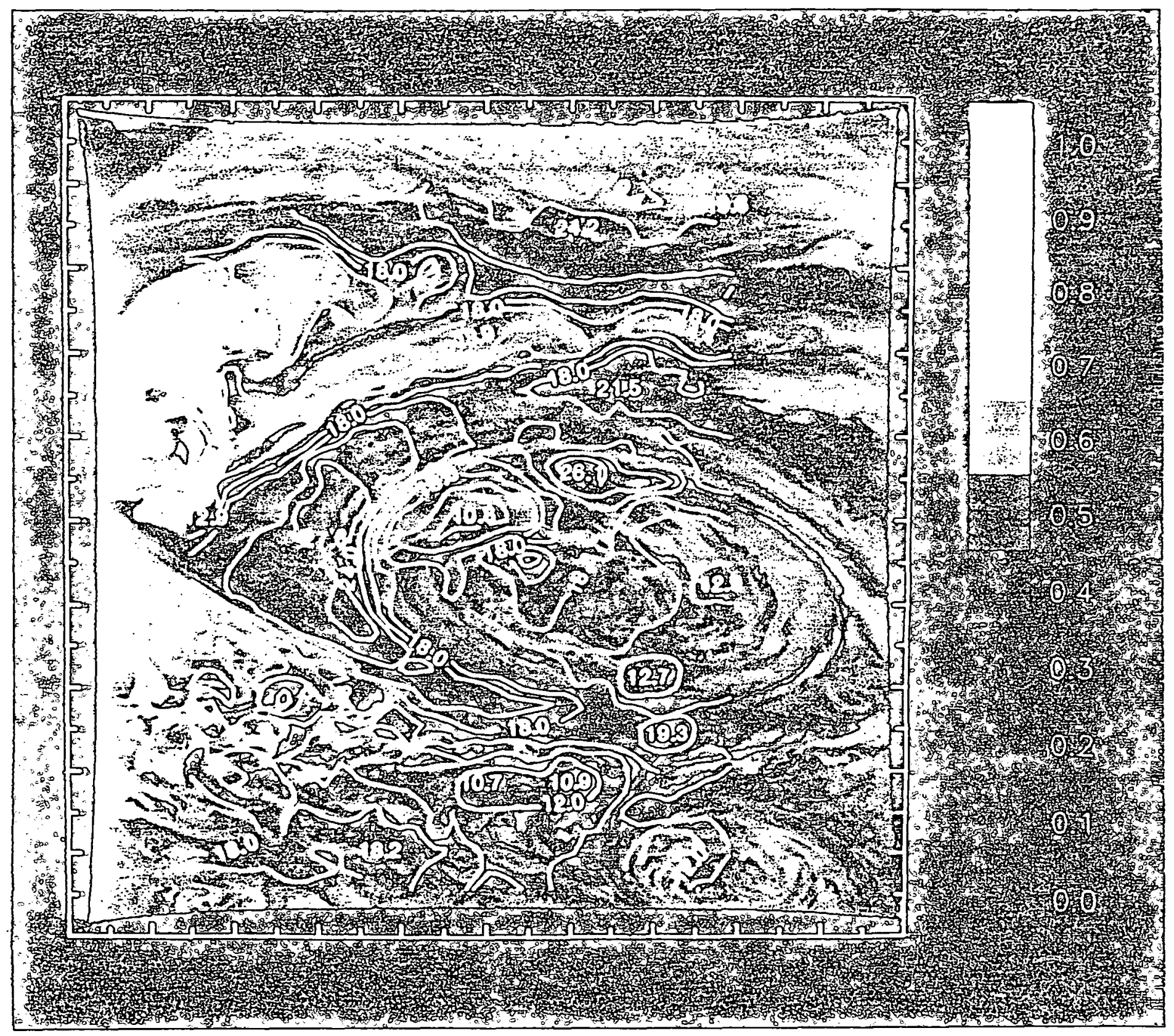




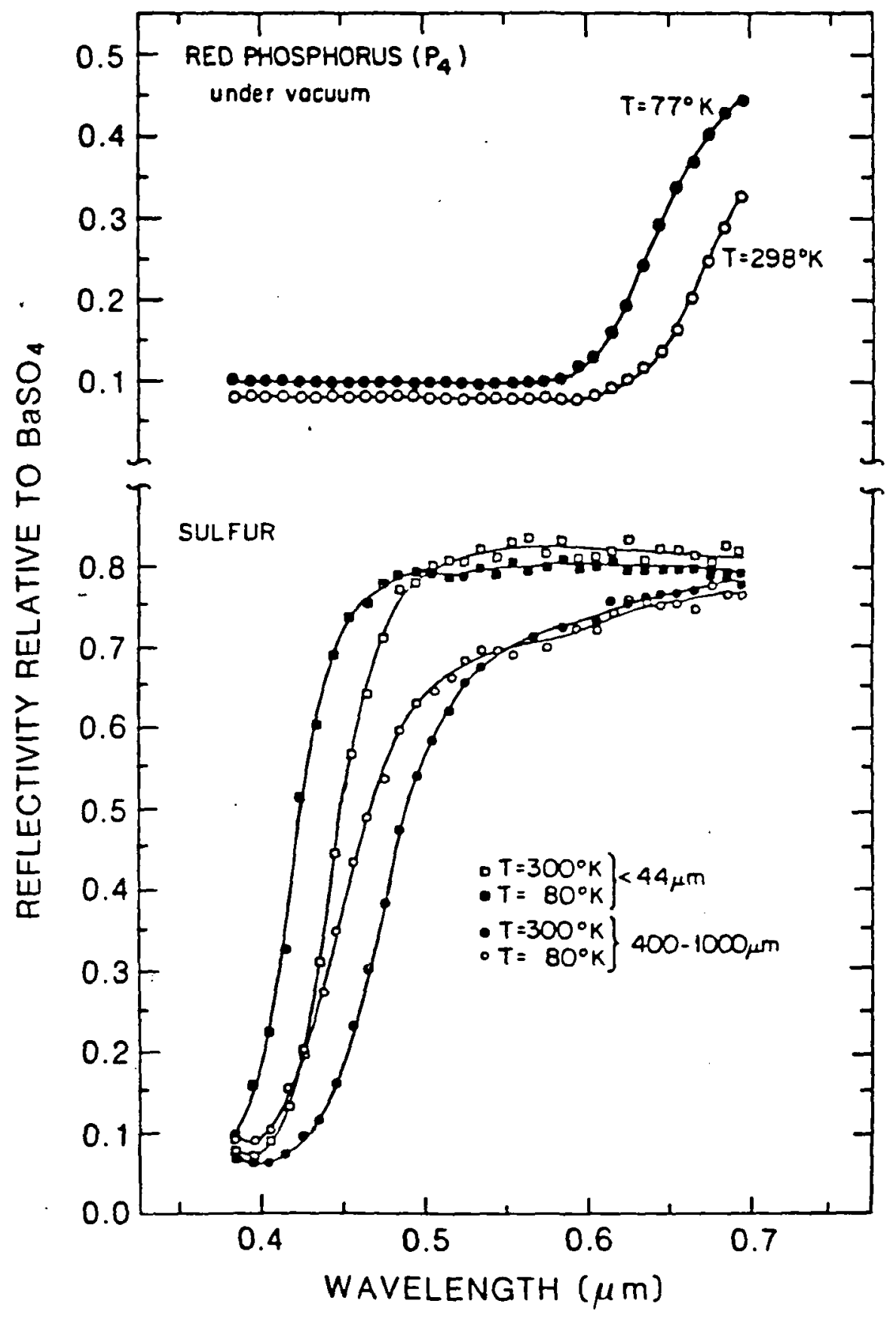

Figure 20 


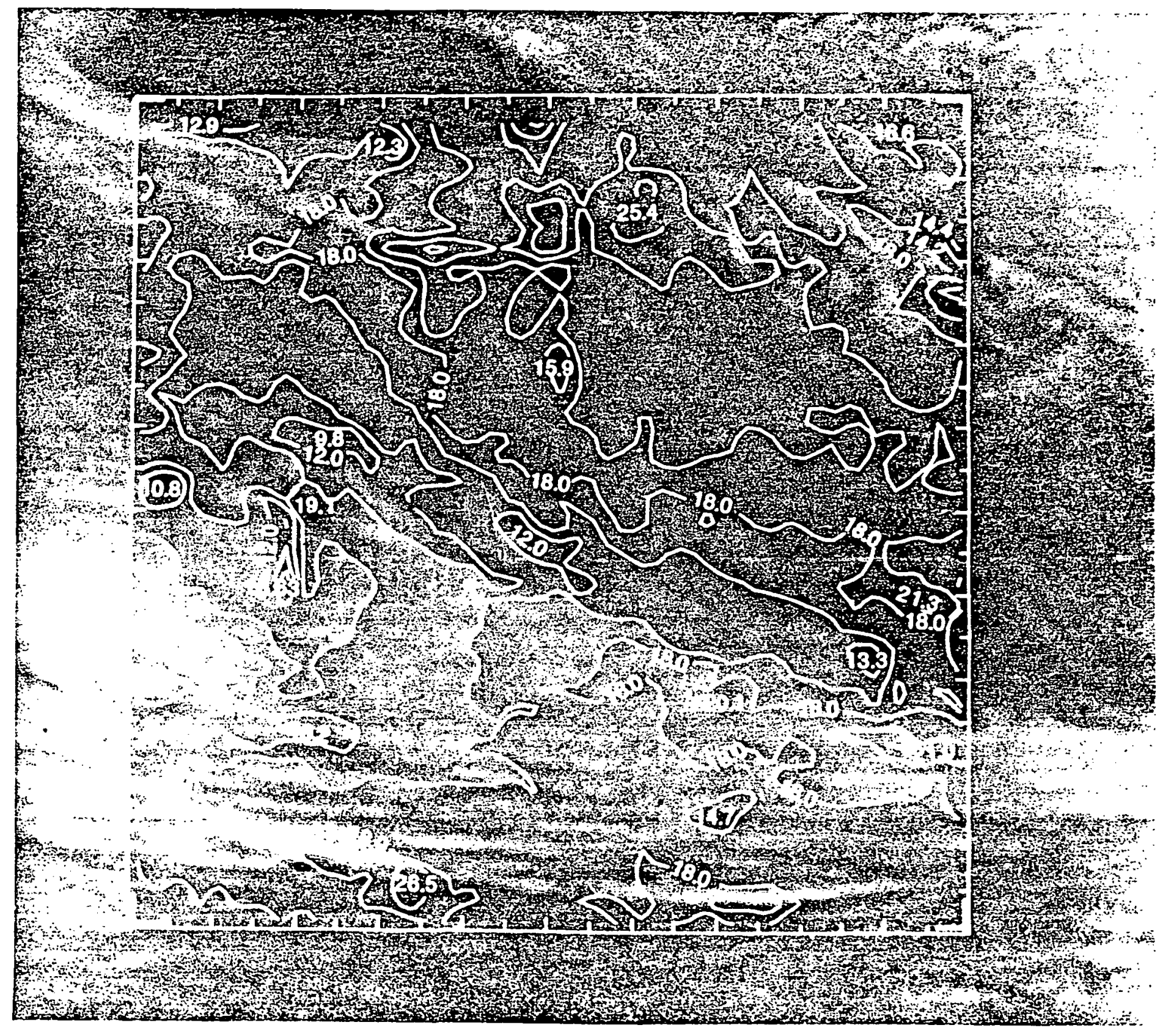




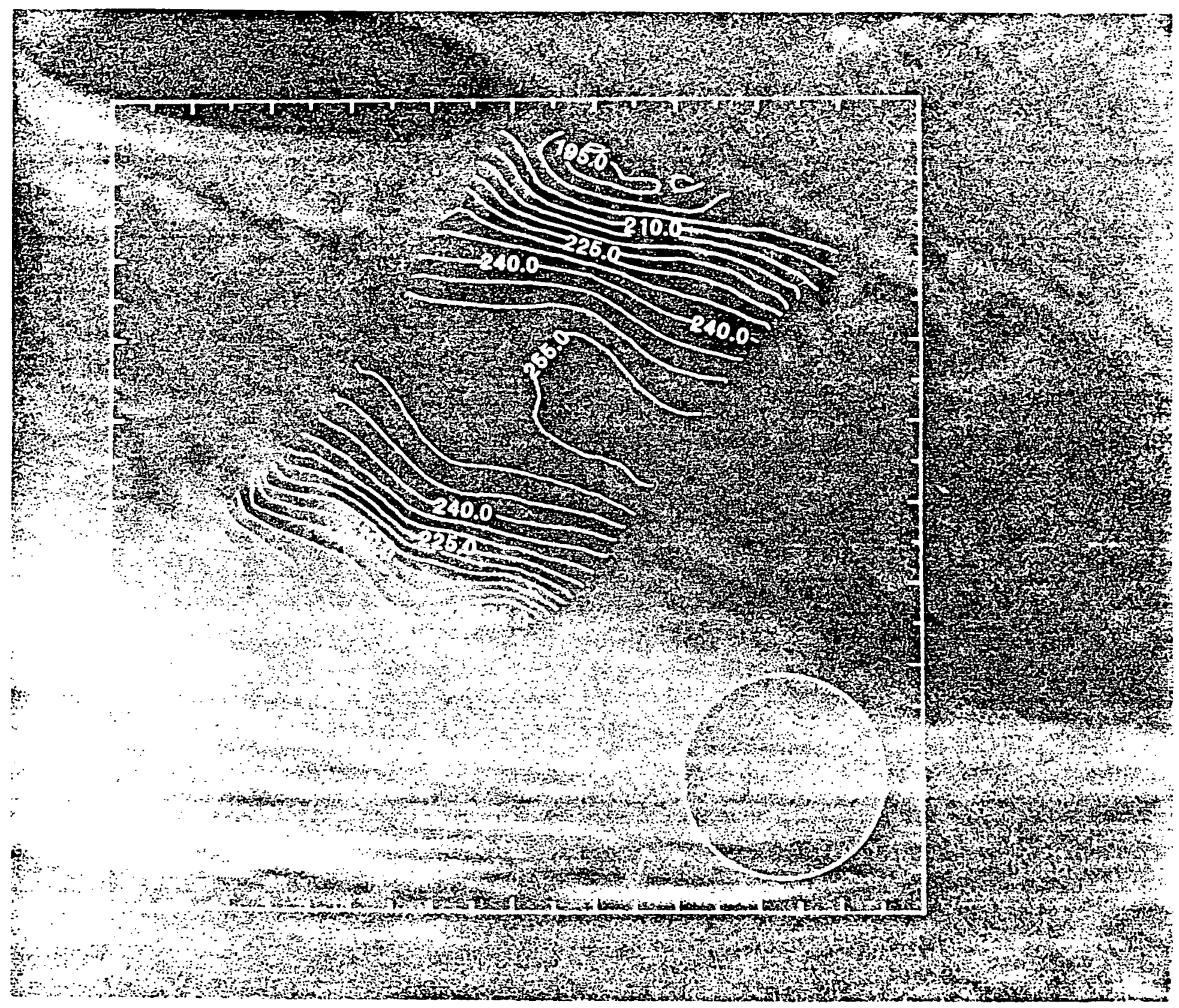




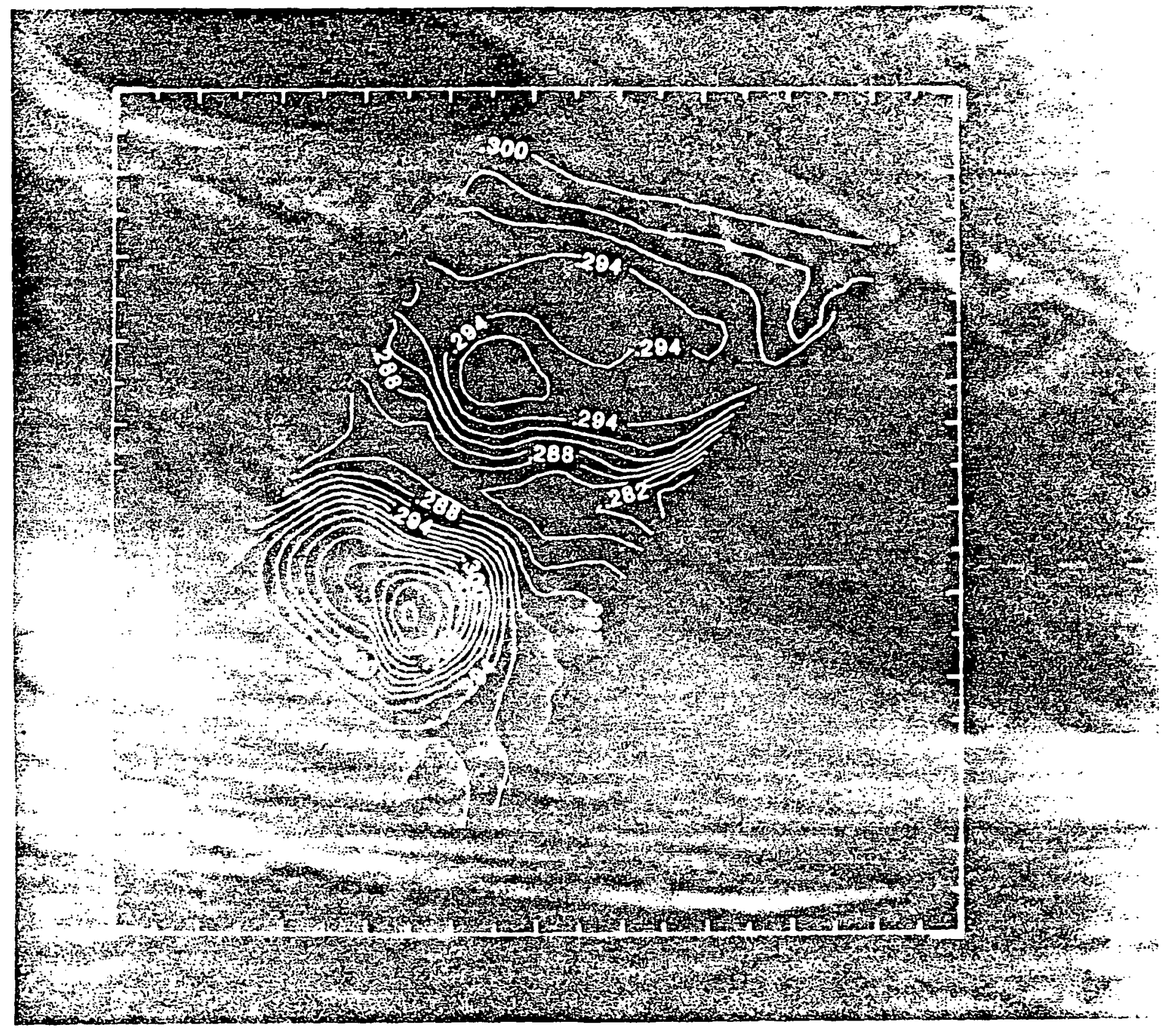




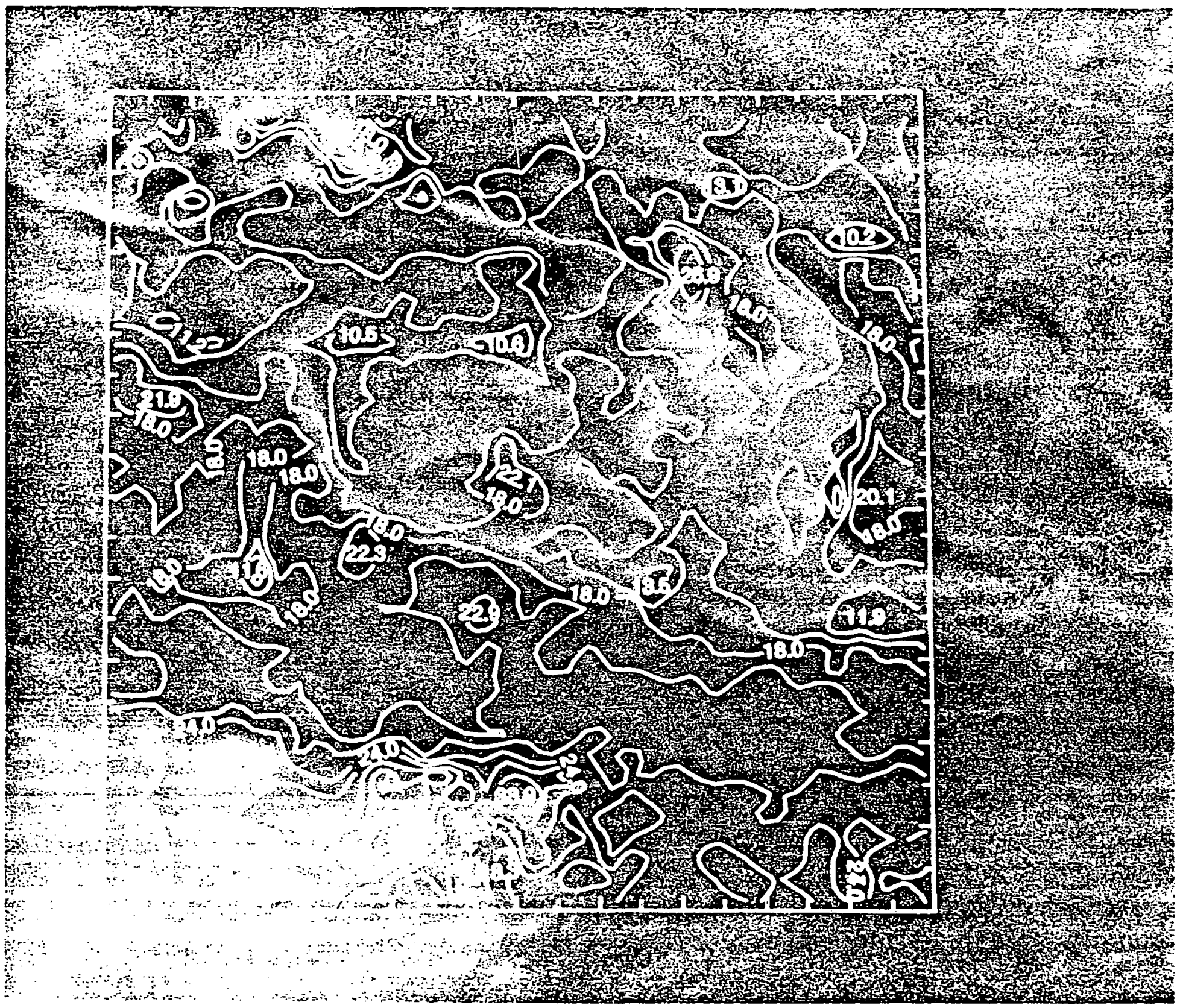




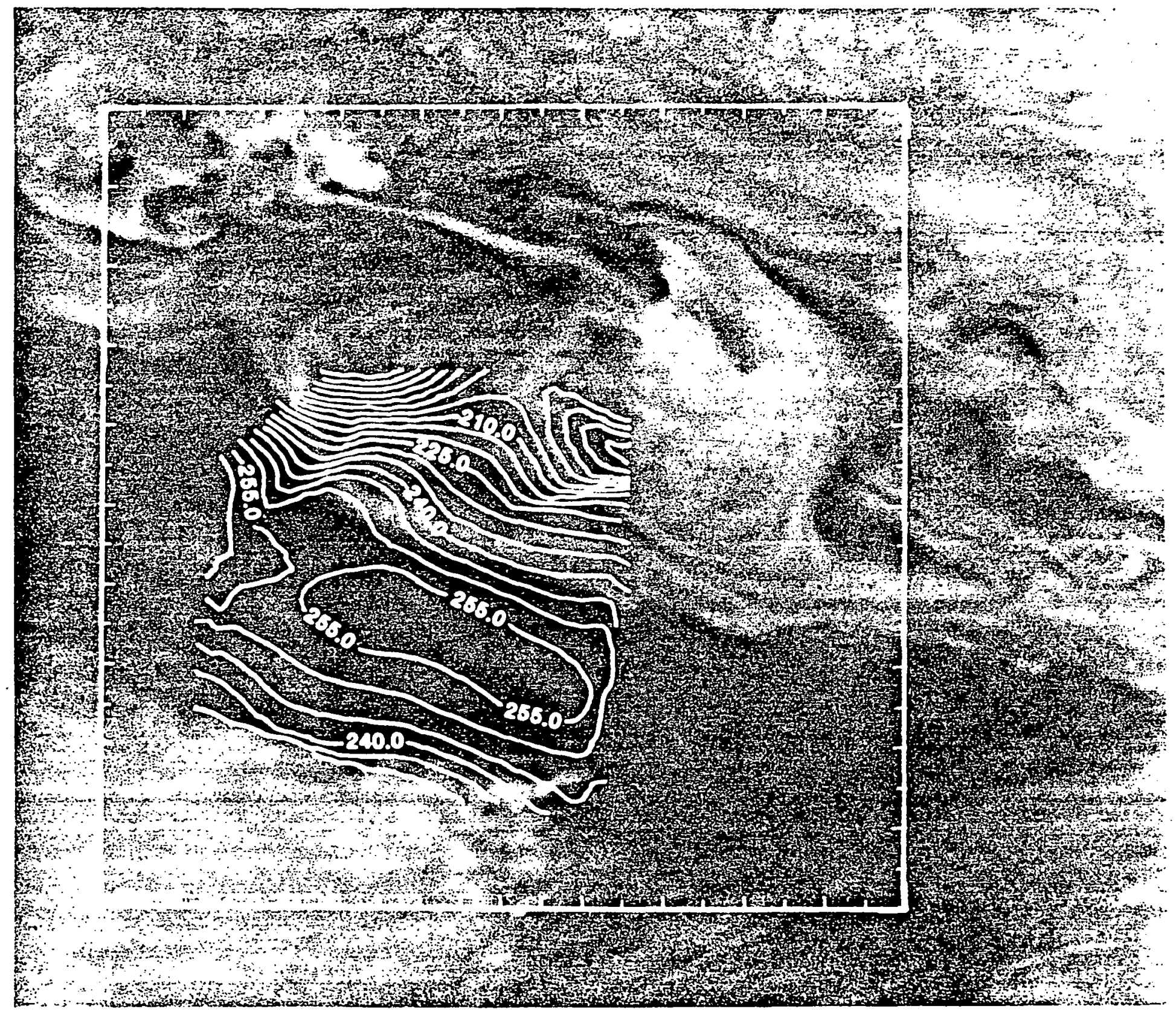




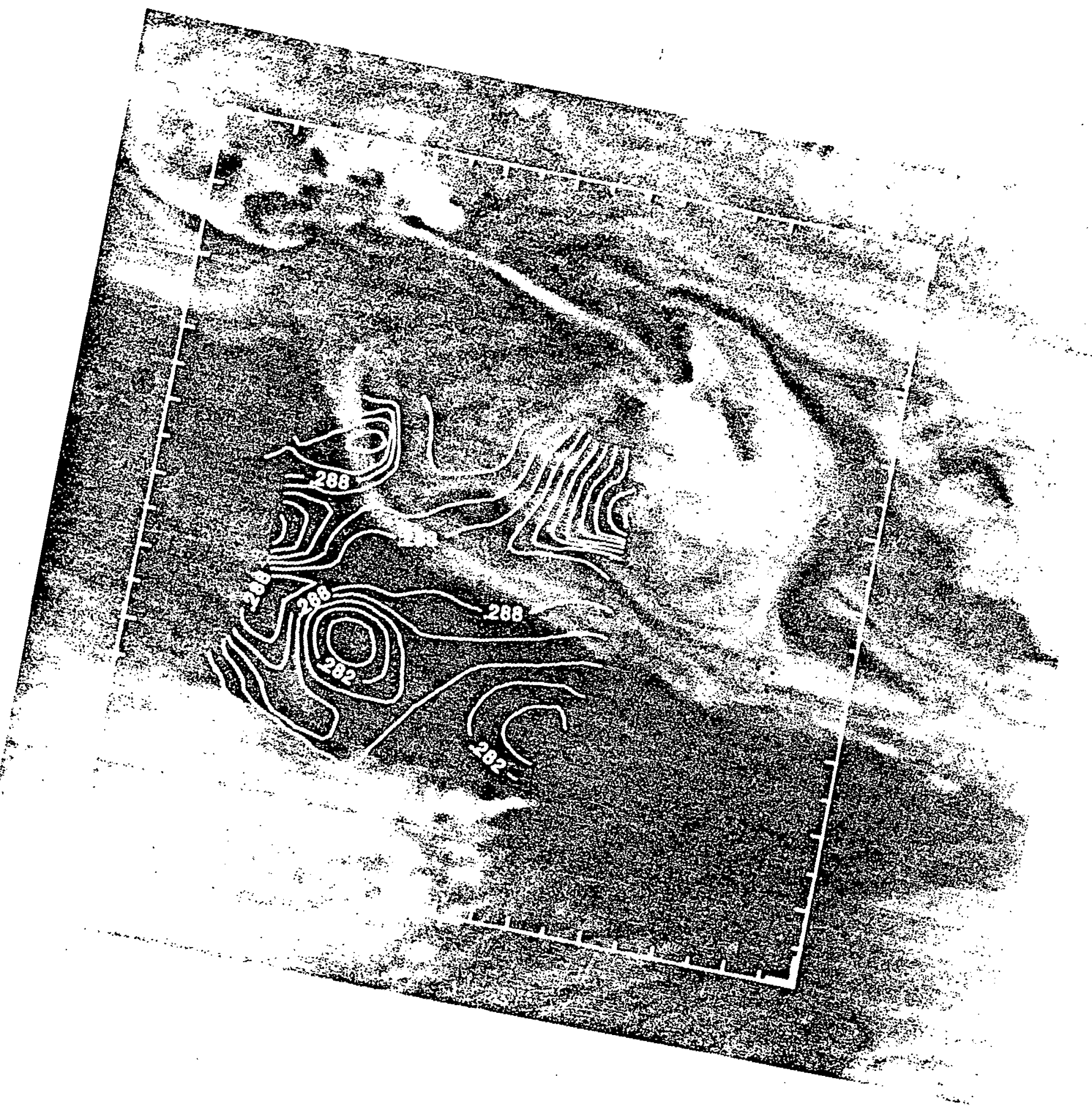




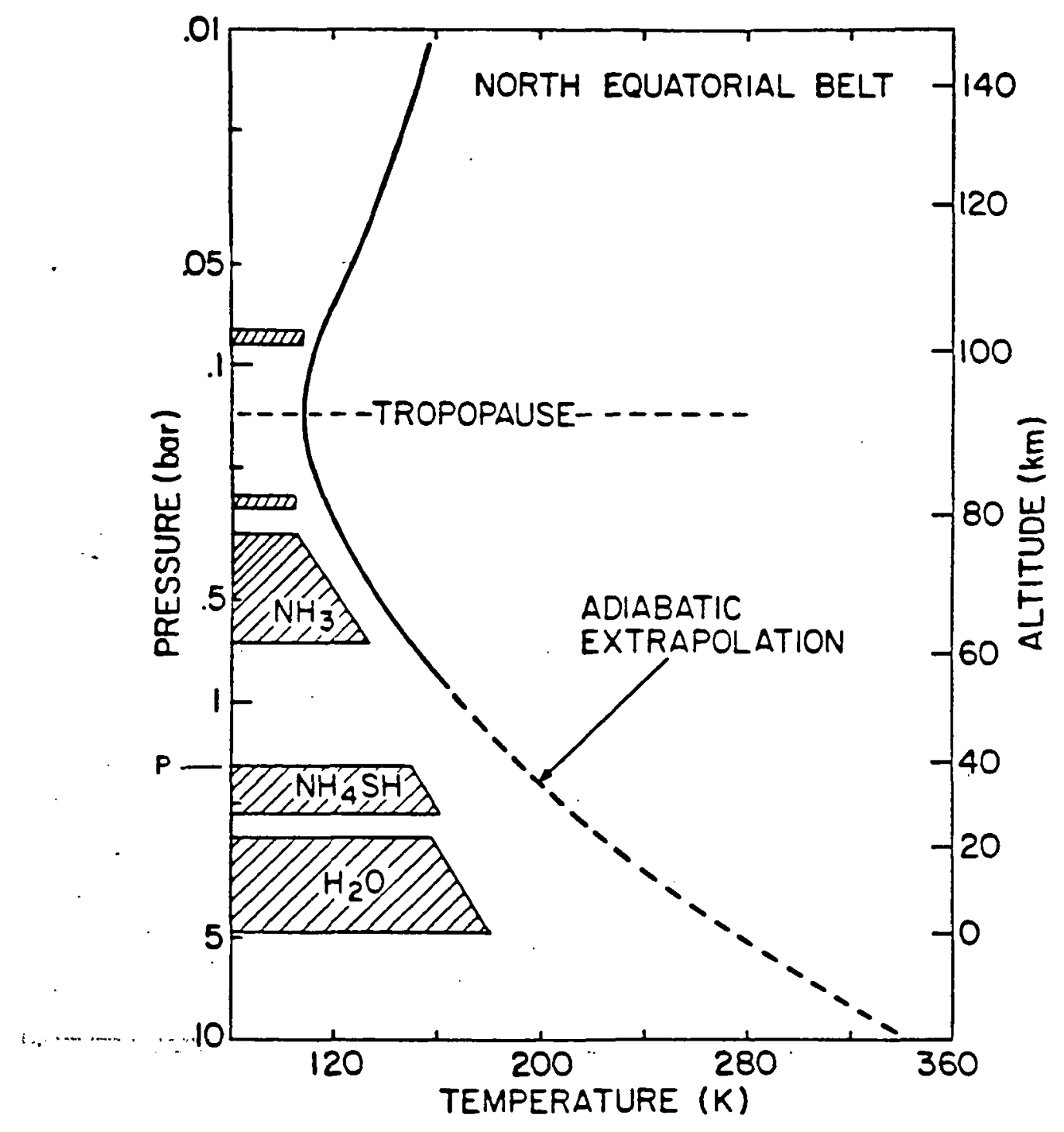

Figure 27 


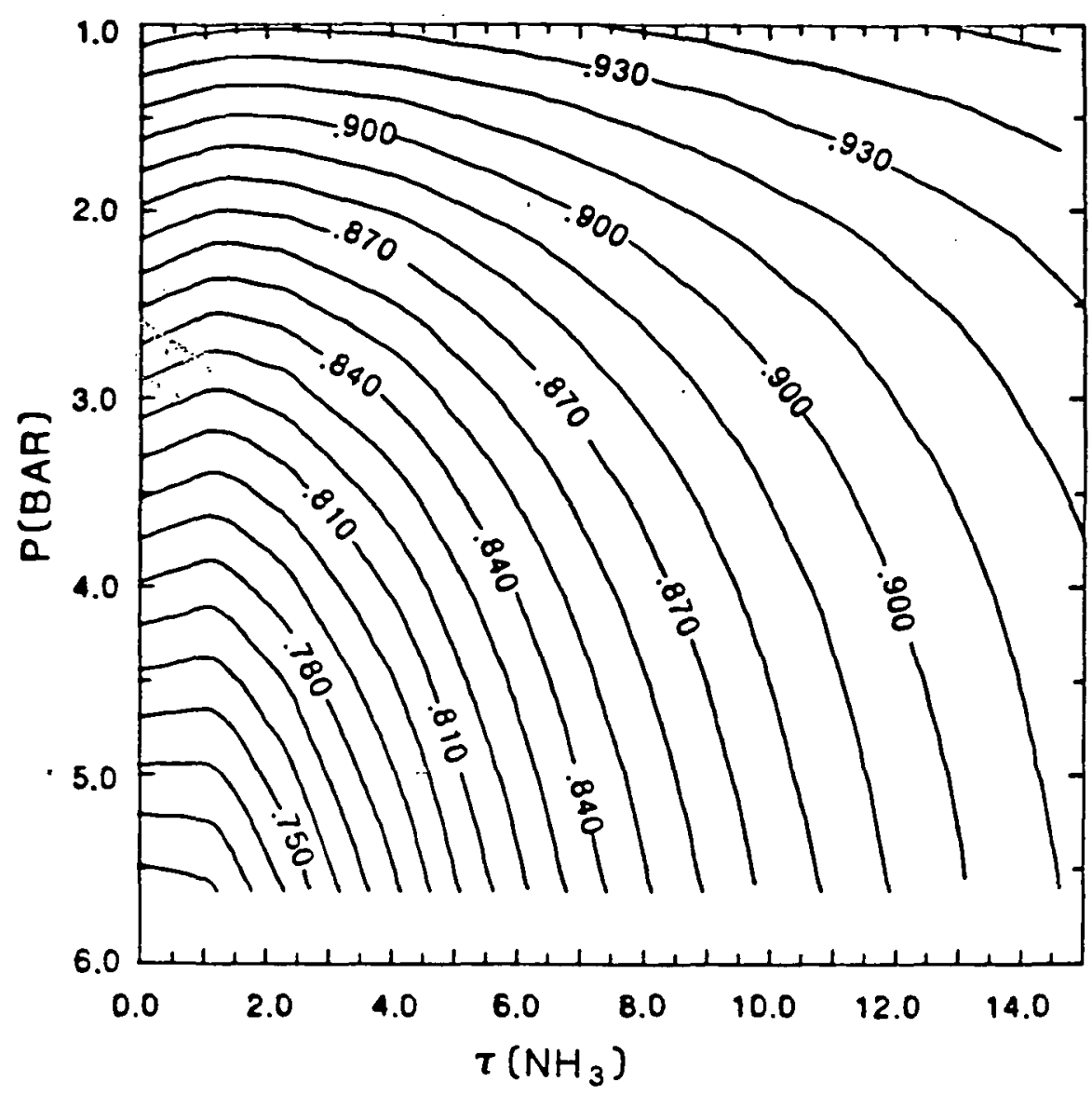

Figure 28

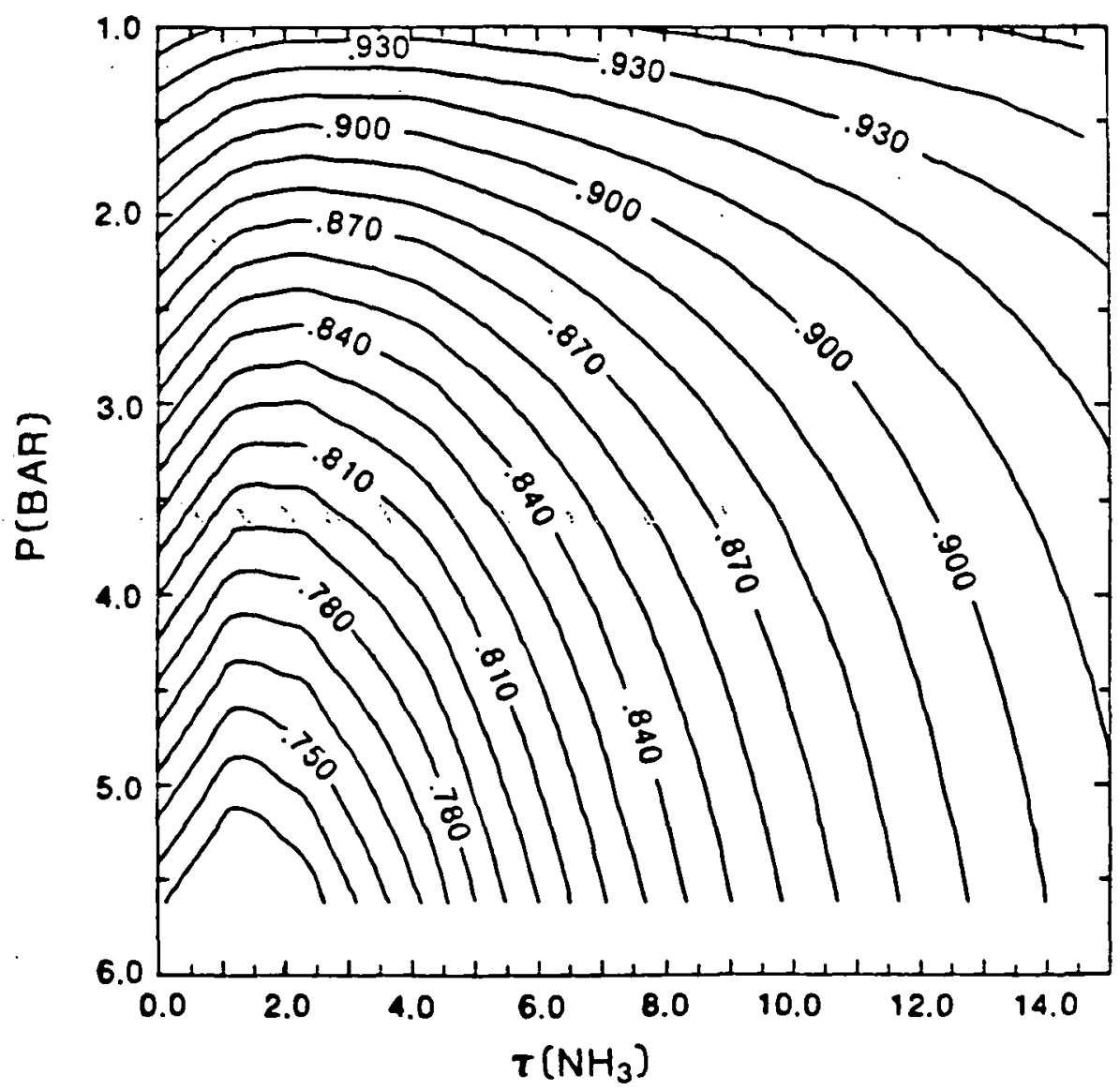

Figure 29 


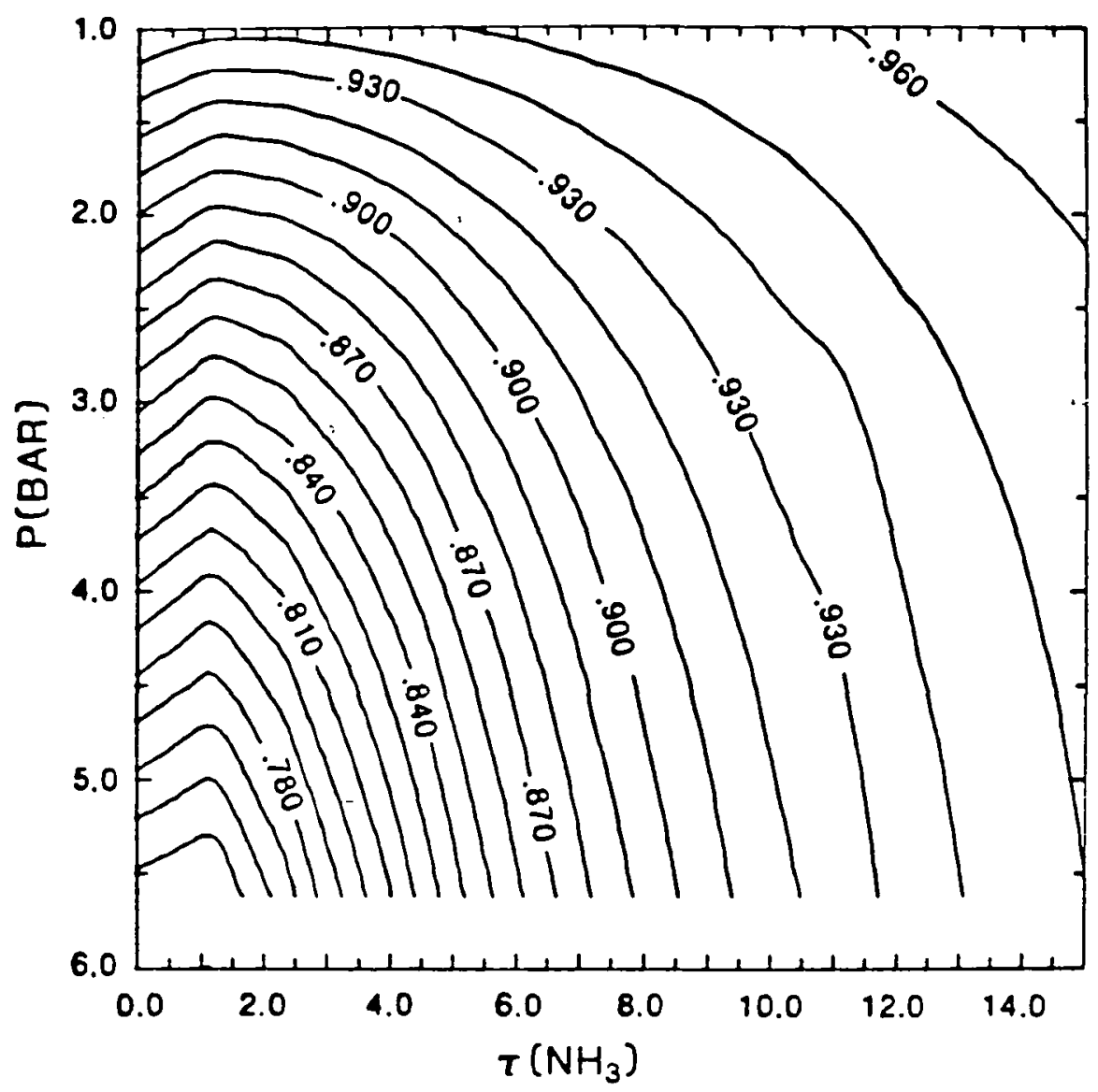

Figure 30

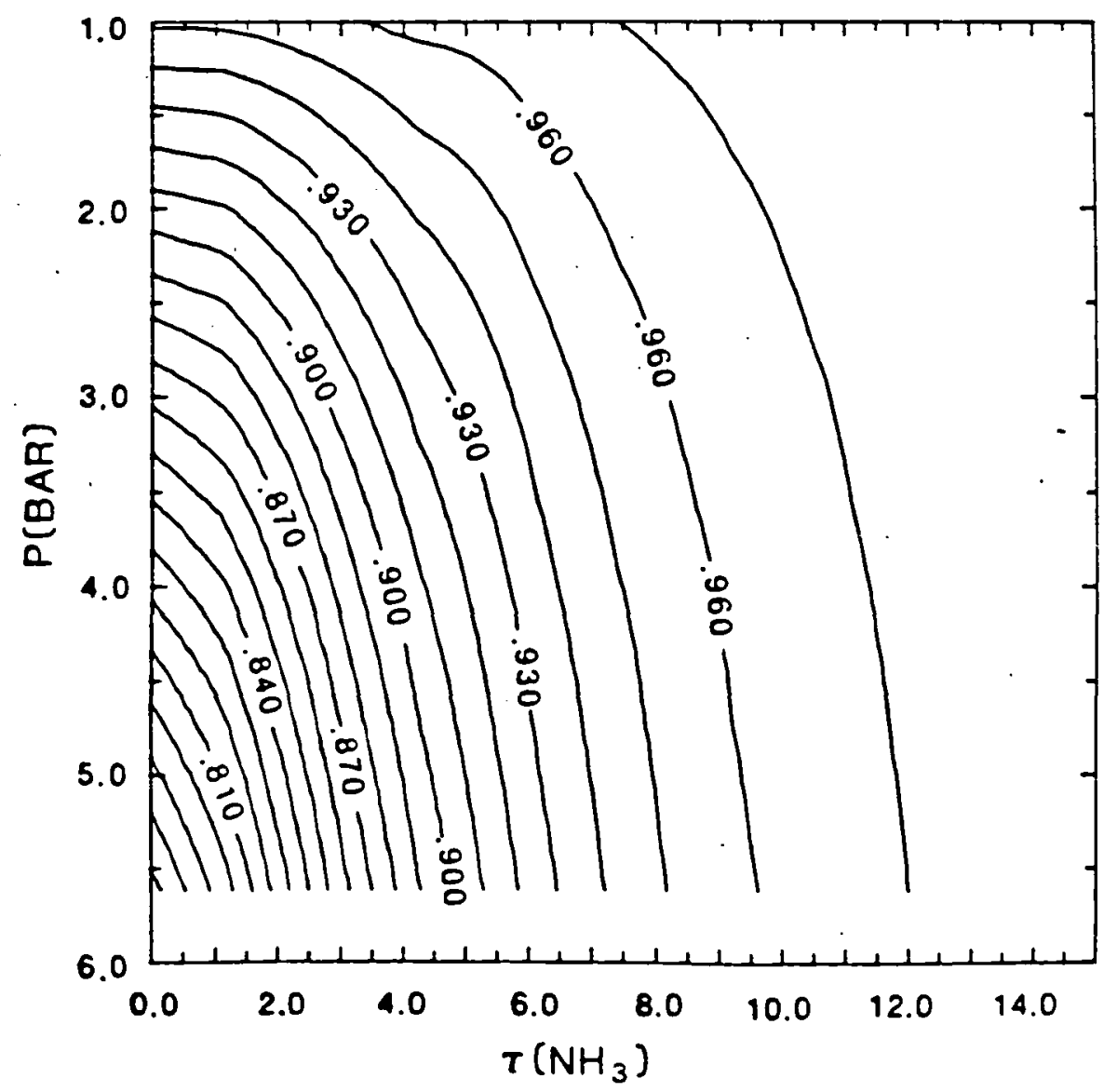

Figure 31 


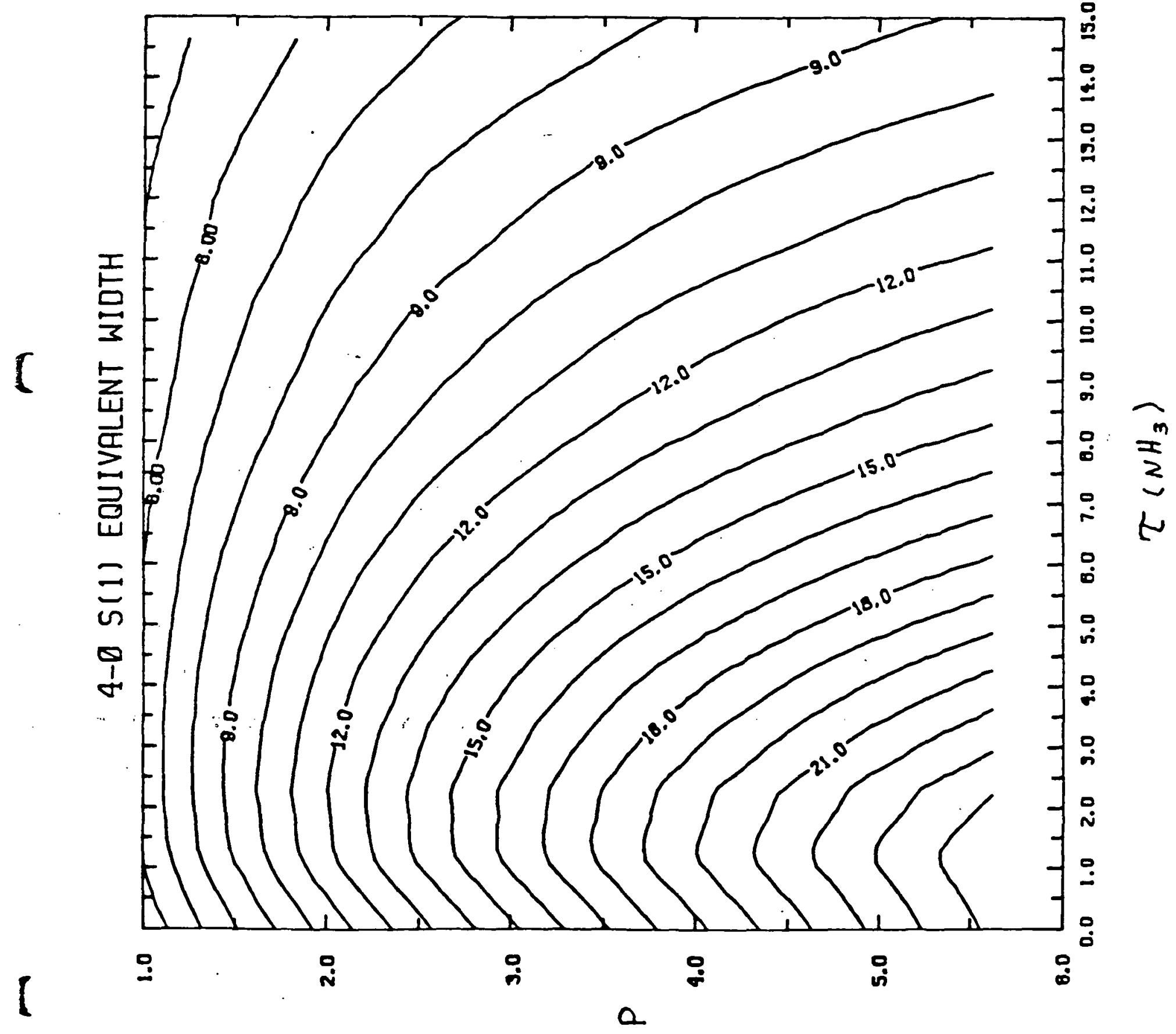

Fig. 32 . 


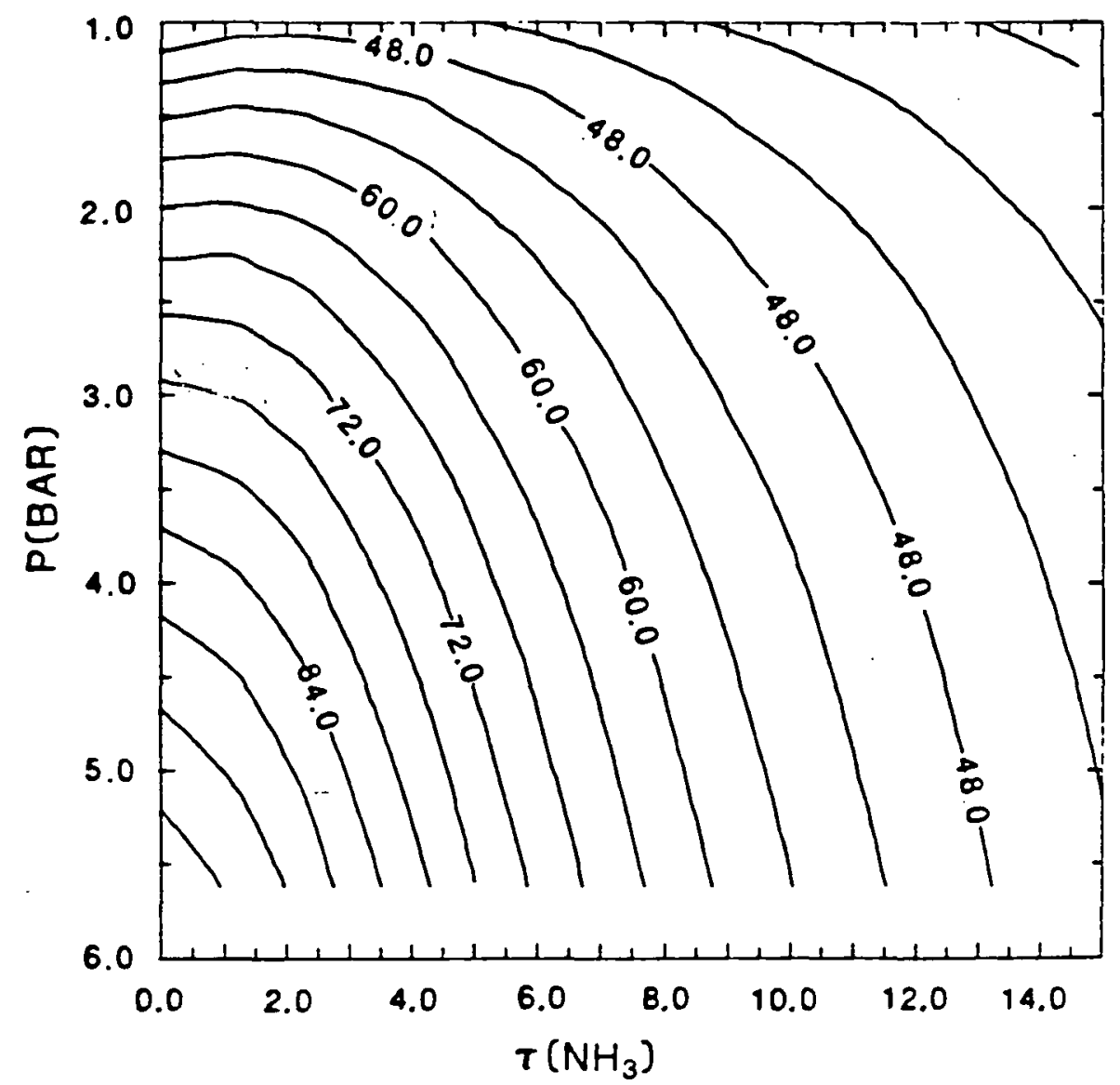

Figure 33

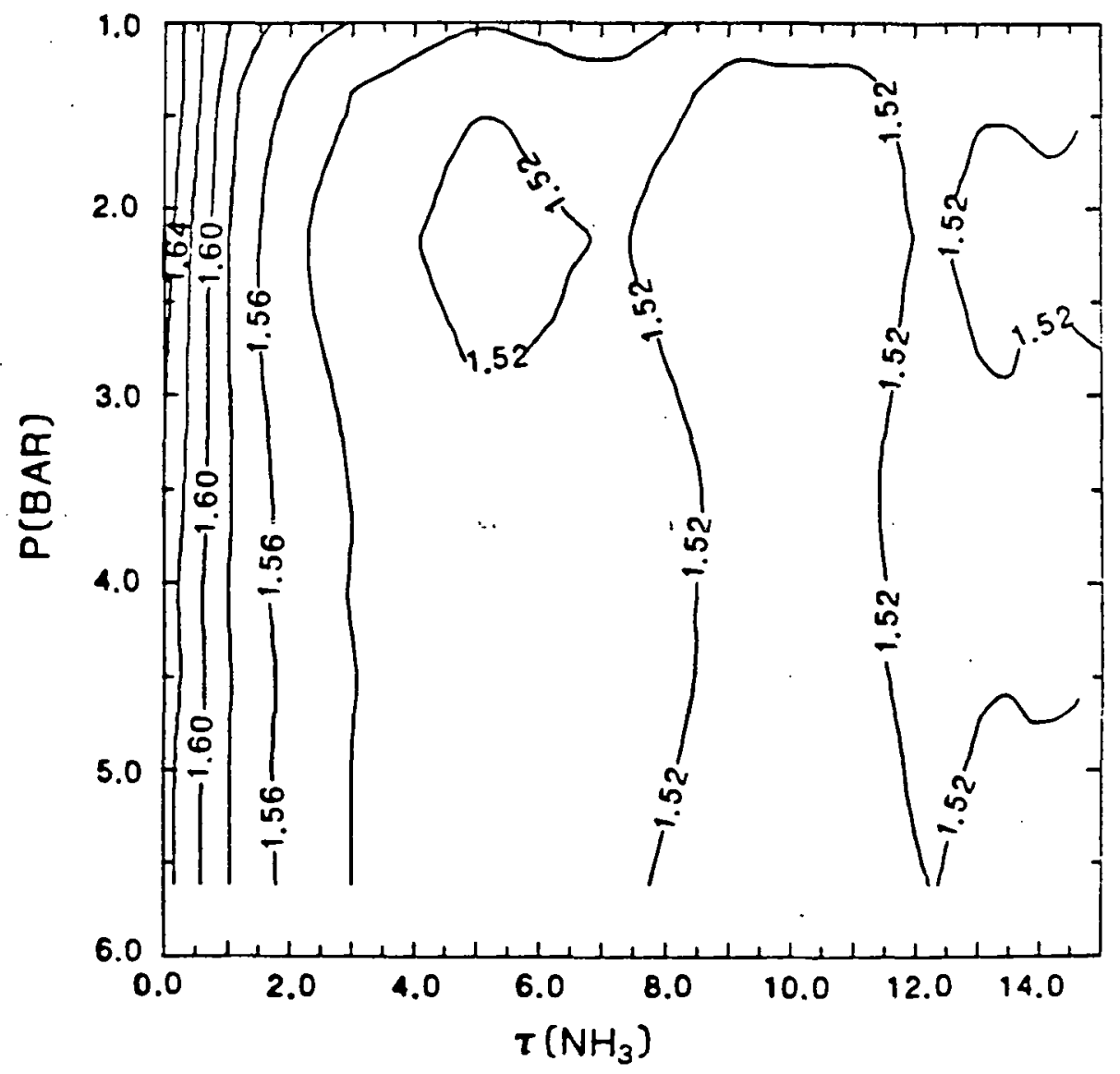




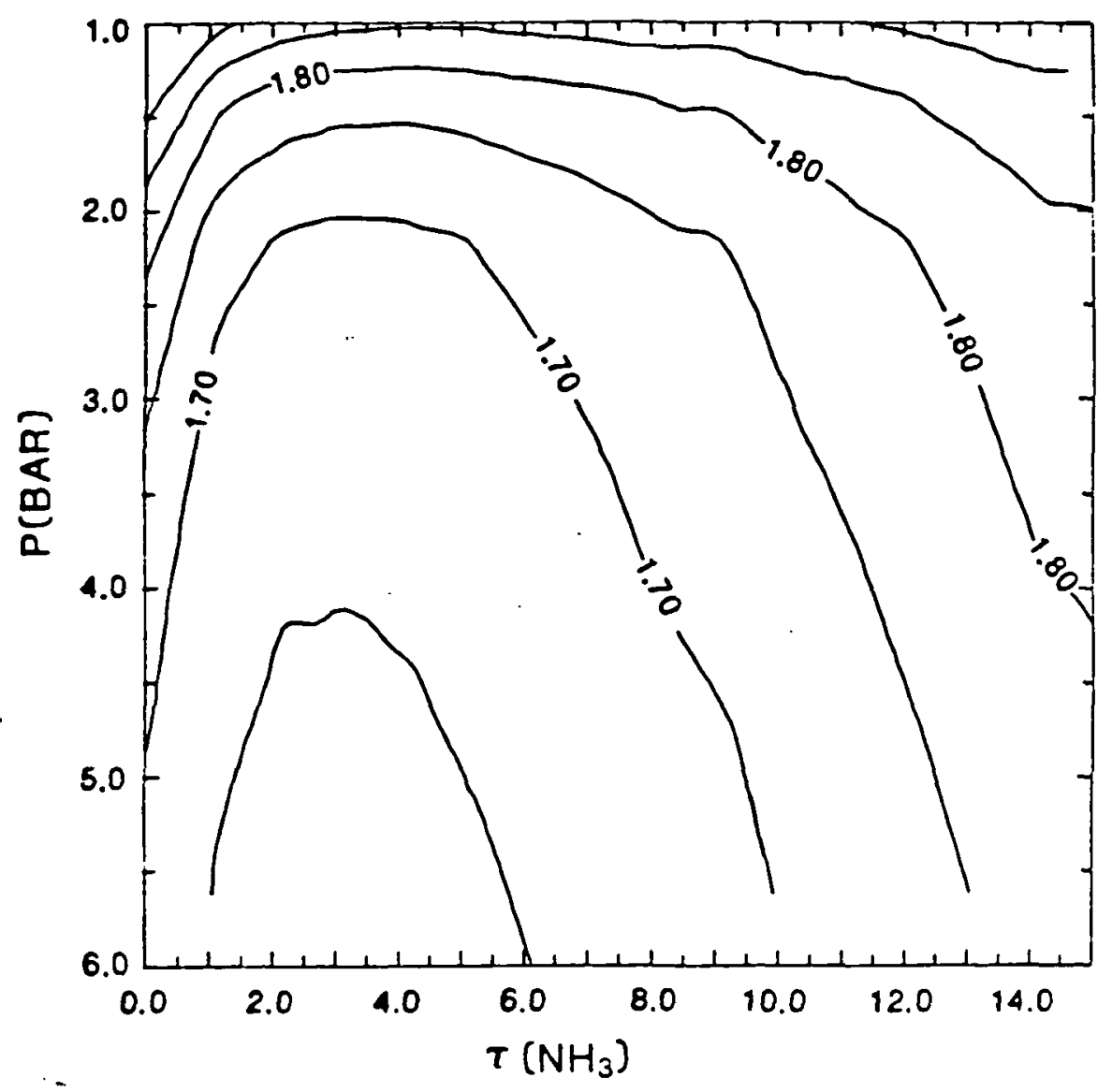

Figure 35

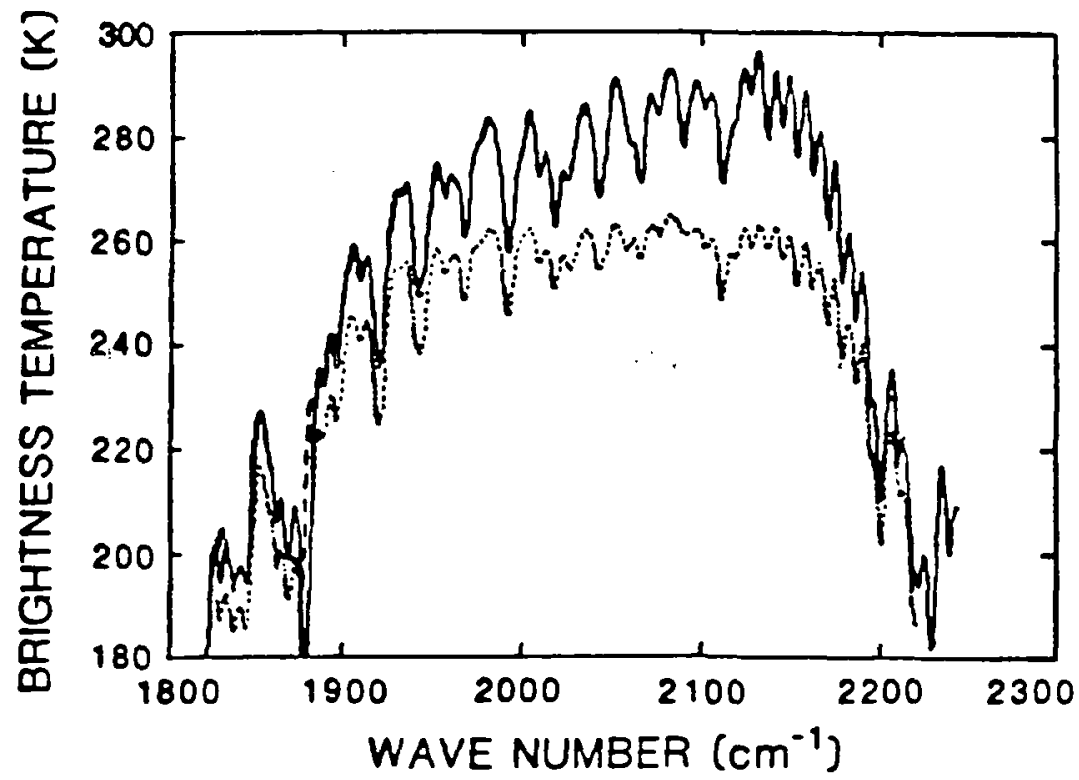

Figure 36 
$\therefore$

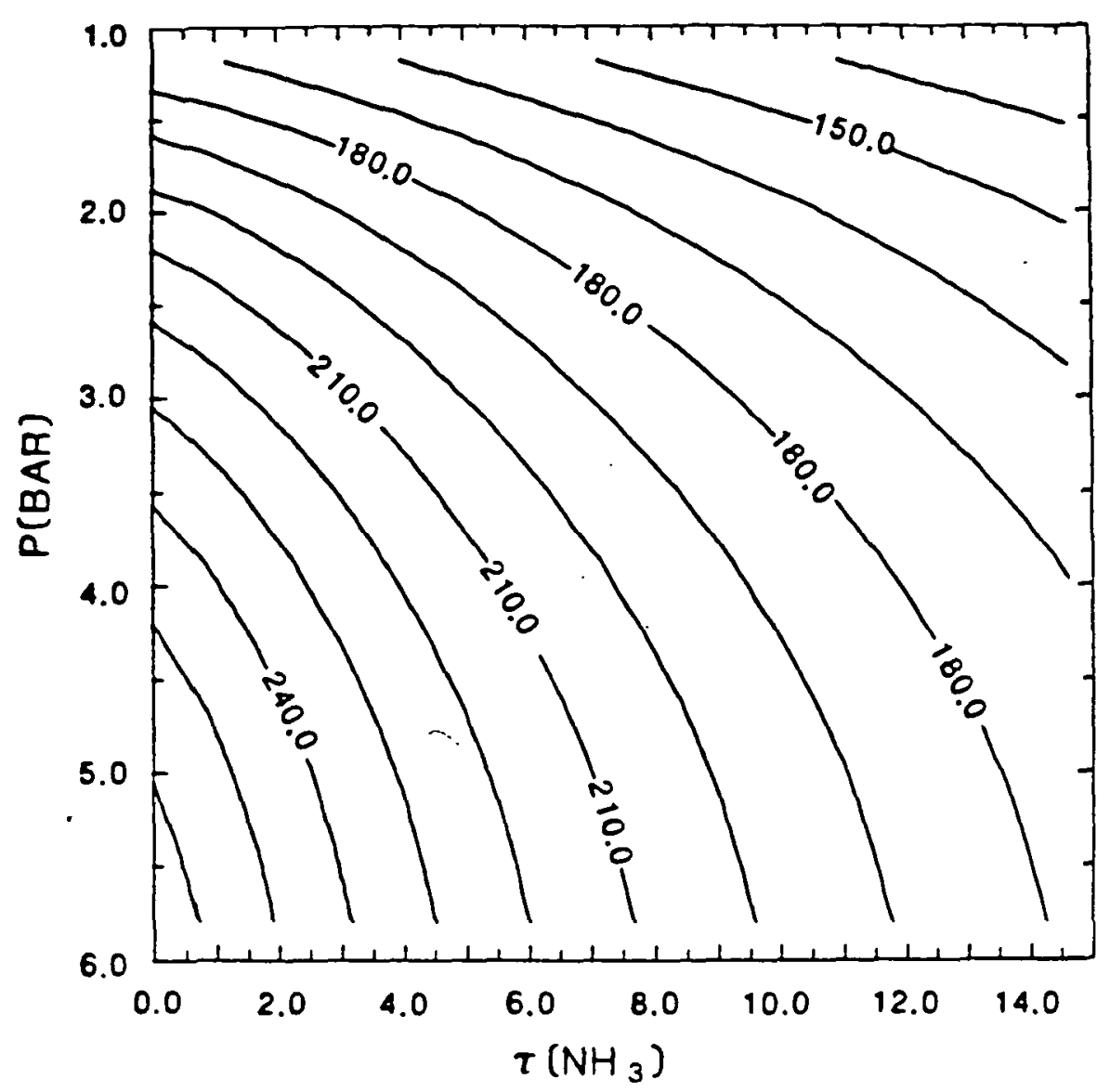

Figure 37

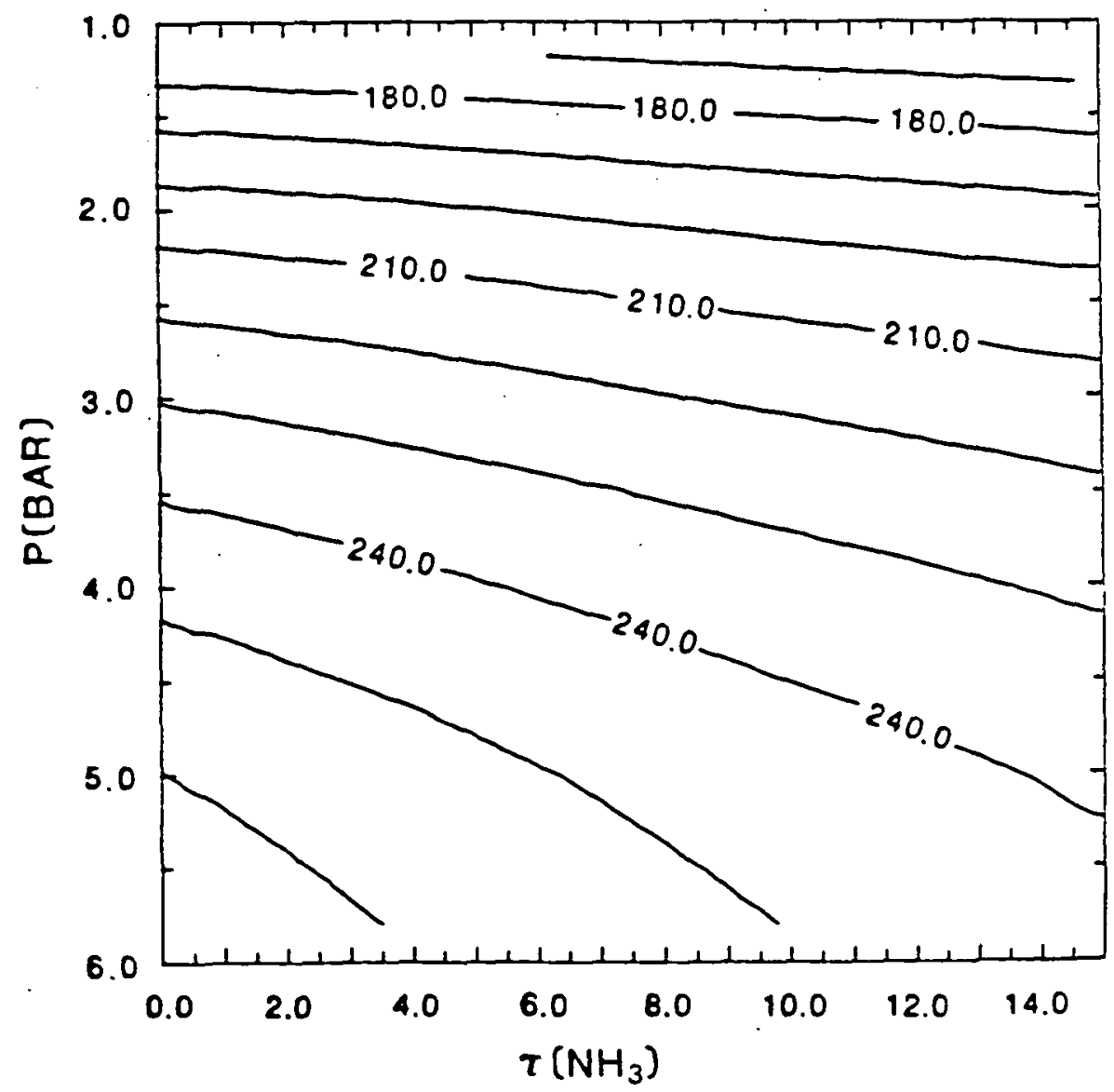

Figure 38 